Supporting Information for

\title{
Soft Mode Metal-Linker Dynamics in Carboxylate MOFs Evidenced by Variable-Temperature Infrared Spectroscopy
}

\author{
Anastasia B. Andreeva, Khoa N. Le, Lihaokun Chen, \\ Michael E. Kellman, Christopher H. Hendon*, Carl K. Brozek* \\ Department of Chemistry and Biochemistry, Materials Science Institute, \\ University of Oregon, Eugene, OR 97403 \\ Email: chendon@uoregon.edu and cbrozek@uoregon.edu
}

\section{Table of Contents}

$\begin{array}{ll}\text { Experimental methods } & \text { S2 }\end{array}$

$\begin{array}{ll}\text { MOF syntheses } & \text { S3 }\end{array}$

$\begin{array}{ll}\text { DRIFTS Section I } & \text { S11 }\end{array}$

$\begin{array}{lr}\text { DRIFTS Section II } & \text { S24 }\end{array}$

$\begin{array}{lr}\text { Computational methods } & \text { S26 }\end{array}$

$\begin{array}{lr}\text { Overview of Models } & \text { S48 }\end{array}$

$\begin{array}{ll}\text { Morse-Potential Model } & \text { S49 }\end{array}$

$\begin{array}{lr}\text { Cross-anharmonicity Model } & \text { S50 }\end{array}$

$\begin{array}{ll}\text { Equilibrium Model } & \text { S51 }\end{array}$

$\begin{array}{ll}\text { References } & \text { S52 }\end{array}$ 


\section{Experimental Methods}

Materials. All commercial chemicals were used as received unless stated otherwise. Copper (II) nitrate trihydrate (99\%, Acros Organics), terephthalic acid (98\%, Sigma Aldrich), and $N, N$ dimethylformamide (DMF, ACS grade, Fisher Scientific), methylene chloride (DCM, HPLC grade, Oakwood Chemical), 1,3,5-benzenetricarboxylic acid (trimesic acid, 98\%, TCI America), ethanol (100\%, Decon), titanium (IV) ethoxide (99.99\%, Strem Chemicals), hexanes (n-hexane, $45 \%$, Oakwood Chemical), titanium (IV) isopropoxide (97\%, Sigma Aldrich), 2aminoterephthalic acid (99\%, Alfa Aesar), calcium chloride dihydrate (Mallinckrodt), glacial acetic acid (ACS grade, Fisher Scientific), manganese (II) chloride tetrahydrate (ACS grade, Baker), zinc (II) nitrate hexahydrate (98\%, Sigma Aldrich), magnesium nitrate hexahydrate (ACS grade, Baker), 2,5-dihydroxyterephthalic acid (97\%, Alfa Aesar), zirconium (IV) chloride (99.5\% trace metals basis, Beantown Chemical), concentrated hydrochloric acid (37\%, ACS grade, Sigma Aldrich). Solvothermal syntheses were conducted in 100-mL Duran Schott bottles or autoclave PAAR bombs in Yamato Convection oven unless stated otherwise.

Characterization. Sample purity was verified by powder X-Ray diffraction (PXRD) with a Bruker D2 Phaser benchtop diffractometer. Variable-temperature diffuse reflectance infrared Fourier transform spectroscopy (DRIFTS) was performed on a Nicolet 6700 FT-IR spectrometer using a Transmission E.S.P. attachment paired with Harrick Scientific Praying Mantis Diffuse Reflection (DRP) accessory and MCT detector. A Harrick Scientific Low Temperature Reaction Chamber (CHC) that enabled introducing vacuum to the sample was equipped with UV quartz and 2 Harrick $15 \times 2 \mathrm{~mm}$ potassium bromide windows. In order to maximize intensity, all samples were diluted with ground KBr. Reflectance FTIR spectra were collected in the range of $4000-650 \mathrm{~cm}^{-1}$ with $1.9280 \mathrm{~cm}^{-1}$ resolution and 32 scans unless stated otherwise. 


\section{MOF Syntheses}

HKUST-1. Following a procedure adapted from Van Goethem et. al, ${ }^{1}$ trimesic acid $(0.7204 \mathrm{~g}, 3.43$ mmol) and $N, N$-dimethylformamide (DMF, $8 \mathrm{~mL}$ ) were combined in a jar and sonicated for $\sim 5$ minutes. Separately, copper (II) nitrate trihydrate $(4.0201 \mathrm{~g}, 16.64 \mathrm{mmol})$ was dissolved in a mixture of water and ethanol $(1: 1 ; 8: 8 \mathrm{~mL})$, added to a Teflon-lined Schott jar with the trimesic acid solution, and the resulting mixture was heated at $80{ }^{\circ} \mathrm{C}$ for 24 hours. After cooling to room temperature, the blue powder product was isolated by centrifuging the mixture and decanting the mother liquor. To clean the product, the material was soaked in fresh DMF (15 mL) overnight. On the next day, the material was again isolated by centrifuging the mixture and decanting the solvent. To remove residual DMF, the material was soaked twice over the course of a day in $15 \mathrm{~mL}$ of fresh ethanol. After soaking overnight, the ethanol was decanted, and the product was dried in a vacuum oven overnight at room temperature.

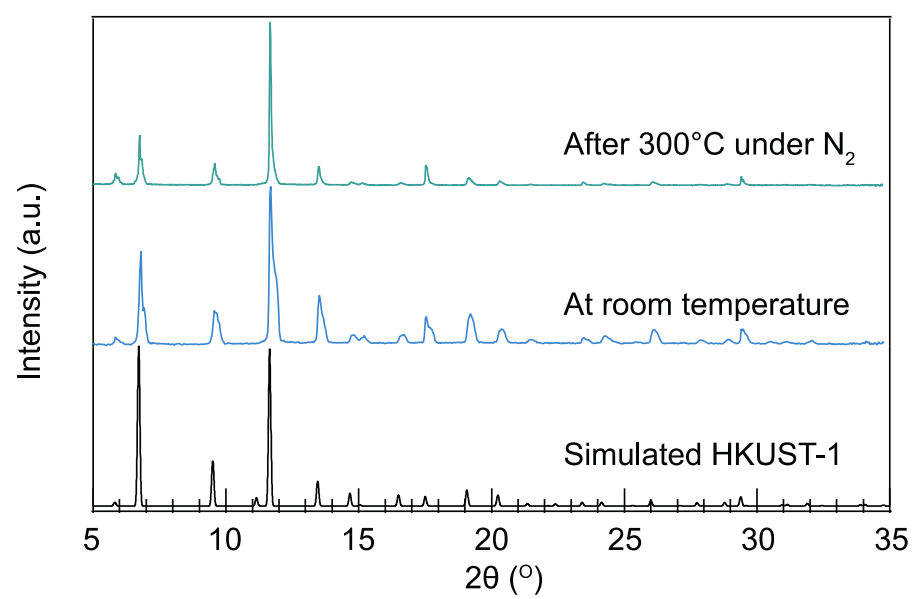

Figure S1: Experimental and simulated powder X-ray diffraction patterns of HKUST-1. 
MUV-10 (Ca). Following a procedure adapted from Castells-Gil et. al, ${ }^{2}$ trimesic acid $(0.125 \mathrm{~g}$, $0.60 \mathrm{mmol})$ and calcium chloride dihydrate $(0.027 \mathrm{~g}, 0.12 \mathrm{mmol})$ were added to a $50-\mathrm{mL}$ Schott bottle and dissolved in DMF (12 mL) under the inert atmosphere of a glove box. The solution was then transferred to a benchtop $\mathrm{N}_{2}$ glovebox, where glacial acetic acid (3.5 $\mathrm{mL}$ ) and titanium (IV) isopropoxide $(36 \mu \mathrm{L})$ were added, and the bottle was resealed. Then, the solution was heated in the oven at $120{ }^{\circ} \mathrm{C}$ for 48 hours. The resulting white powder was centrifuged and washed sequentially with clean DMF, water, and then methanol, and then dried under dynamic vacuum overnight at room temperature.

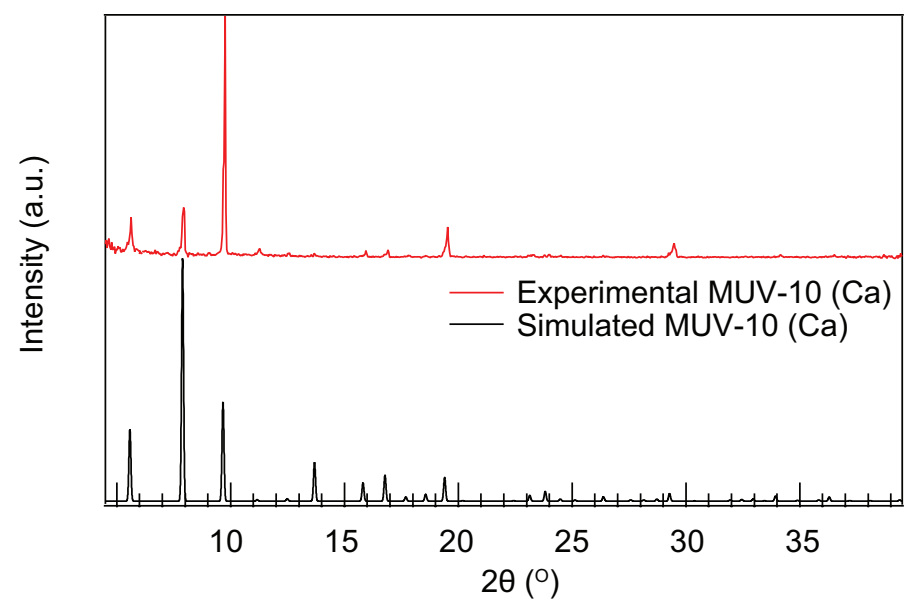

Figure S2: Experimental and simulated powder X-ray diffraction patterns of MUV-10 (Ca). 
MUV-10 (Mn). Following a similar procedure to the synthesis of MUV-10 $(\mathrm{Ca}),{ }^{2}$ trimesic acid $(0.125 \mathrm{~g}, 0.60 \mathrm{mmol})$ and manganese (II) chloride dihydrate $(0.0356 \mathrm{~g}, 0.12 \mathrm{mmol})$ were combined in 50-mL Schott bottle and dissolved in DMF $(12 \mathrm{~mL})$ under inert atmosphere. The solution was transferred to a benchtop $\mathrm{N}_{2}$-filled glovebox, where glacial acetic acid (3.5 mL) titanium (IV) isopropoxide $(36 \mu \mathrm{L})$ were added and the bottle was resealed. The solution was then heated in the oven at $120^{\circ} \mathrm{C}$ for 48 hours. The resulting white powder was centrifuged, washed with clean DMF, water, and methanol, and then dried in a vacuum oven overnight at room temperature.

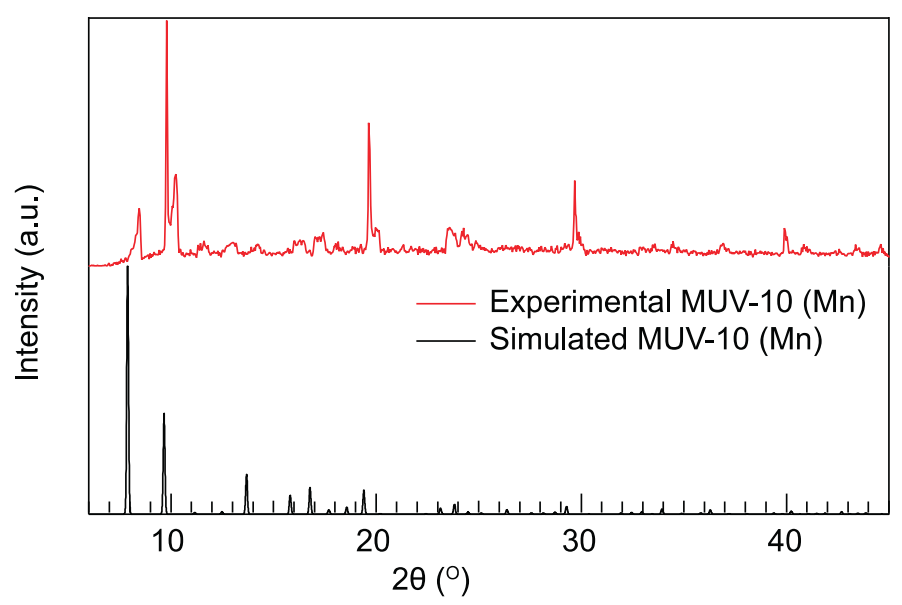

Figure S3: Experimental and simulated powder X-ray diffraction patterns of MUV-10 (Mn).

$M g-M O F-74$. Following a procedure adapted from Yang et. al, ${ }^{3}$ magnesium nitrate hexahydrate (0.6661 g, $2.59 \mathrm{mmol}), 2,5$-dihydroxyterephthalic acid (0.1553 g, $0.78 \mathrm{mmol}), \mathrm{DMF}(60 \mathrm{~mL})$, ethanol (4 mL), and water $(4 \mathrm{~mL})$ were combined under atmospheric conditions in a 250 - $\mathrm{mL}$ Schott jar with a Teflon ring. The mixture was then sonicated until all solids dissolved and heated at 125 ${ }^{\circ} \mathrm{C}$ in a convection oven for 24 hours. The resulting yellow solid was washed and soaked in DMF $(3 \times 15 \mathrm{~mL})$ and ethanol $(3 \times 15 \mathrm{~mL})$ over the course of 2 days and dried in a vacuum oven at room temperature.

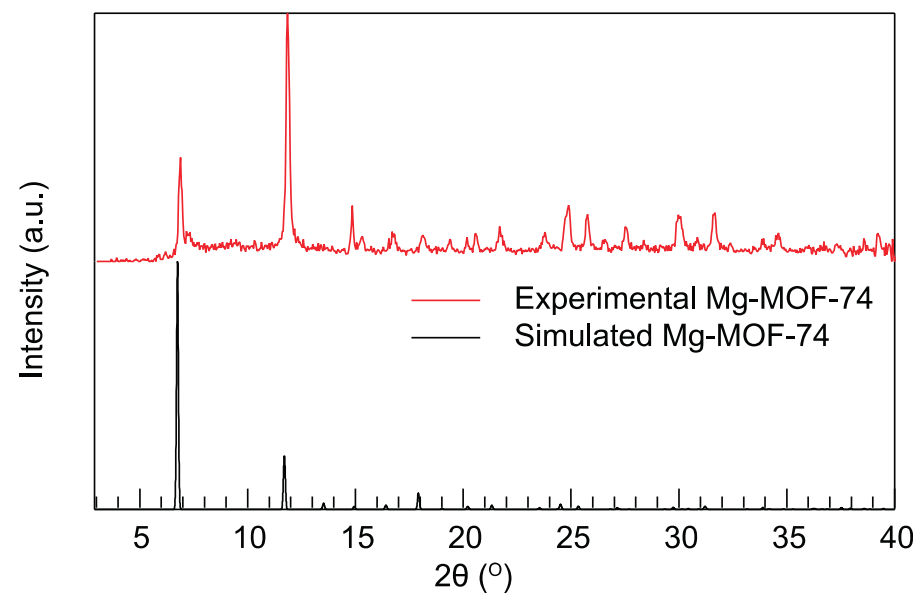

Figure S4: Experimental and simulated powder X-ray diffraction patterns of Mg-MOF-74. 
$\mathrm{Zn-MOF-74.} \mathrm{Following} \mathrm{a} \mathrm{procedure} \mathrm{adapted} \mathrm{from} \mathrm{Choi} \mathrm{et.} \mathrm{al,}{ }^{4}$ zinc (II) nitrate hexahydrate $(0.9038$ g, $3.04 \mathrm{mmol}), 2,5$-dihydroxyterephthalic acid $(0.2066 \mathrm{~g}, 1.04 \mathrm{mmol}), N, N$-dimethylformamide $(40 \mathrm{~mL})$ and isopropanol $(2 \mathrm{~mL})$ were combined under atmospheric conditions in a $100-\mathrm{mL}$ Schott jar with a Teflon ring and sonicated until the solids dissolved. The mixture was then heated in a convection oven for 20 hours at $100^{\circ} \mathrm{C}$. The resulting black solid was washed and soaked in DMF $(3 \times 15 \mathrm{~mL})$ and methanol $(3 \times 15 \mathrm{~mL})$ over the course of 2 days and dried in a vacuum oven at room temperature.

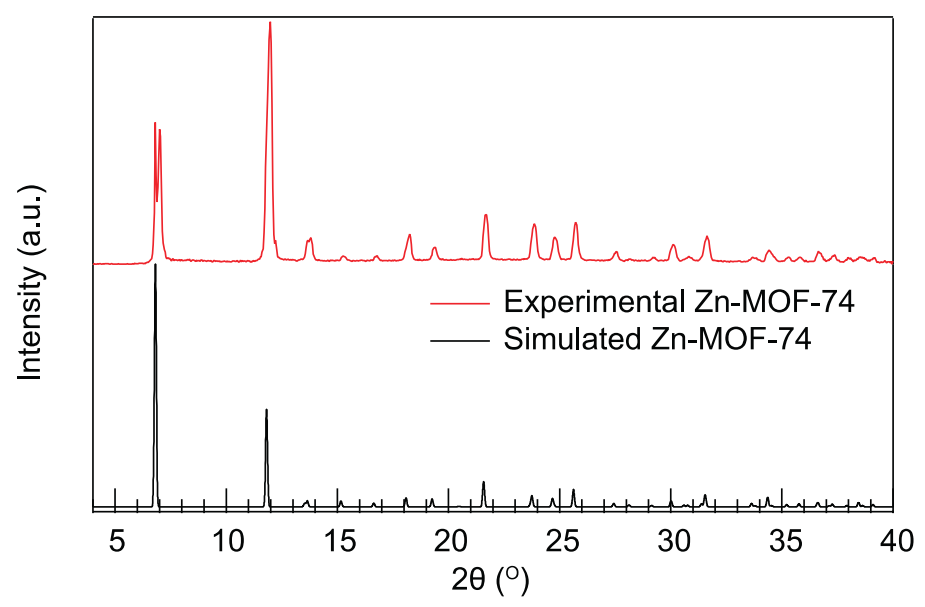

Figure S5: Experimental and simulated powder X-ray diffraction patterns of Zn-MOF-74. 
MOF-5. Following a procedure adapted from Kaye et. al, ${ }^{5}$ zinc (II) nitrate hexahydrate $(0.4531 \mathrm{~g}$, $1.50 \mathrm{mmol})$, terephthalic acid $(0.0830 \mathrm{~g}, 0.50 \mathrm{mmol}), N, N$-dimethylformamide (DMF, $49 \mathrm{~mL})$, and water $(1 \mathrm{~mL})$ were combined in a $100-\mathrm{mL}$ Schott jar with Teflon ring and heated in convection oven for 7 hours at $100{ }^{\circ} \mathrm{C}$. Upon cooling down to room temperature, the resulting large white crystals were isolated by gravity filtration under inert atmosphere in a benchtop glovebox and washed with clean dry DMF ( $20 \mathrm{~mL}$ each). The slurry was transferred to a $\mathrm{N}_{2}$-filled glovebox and washed with fresh DMF over the course of 1 day $(3 \times 20 \mathrm{~mL})$ and with dichloromethane (DCM, $3 \times 20 \mathrm{~mL}$ ) over the course of 3 days. After 3 days, the DCM was decanted, and the product was stored under inert atmosphere.

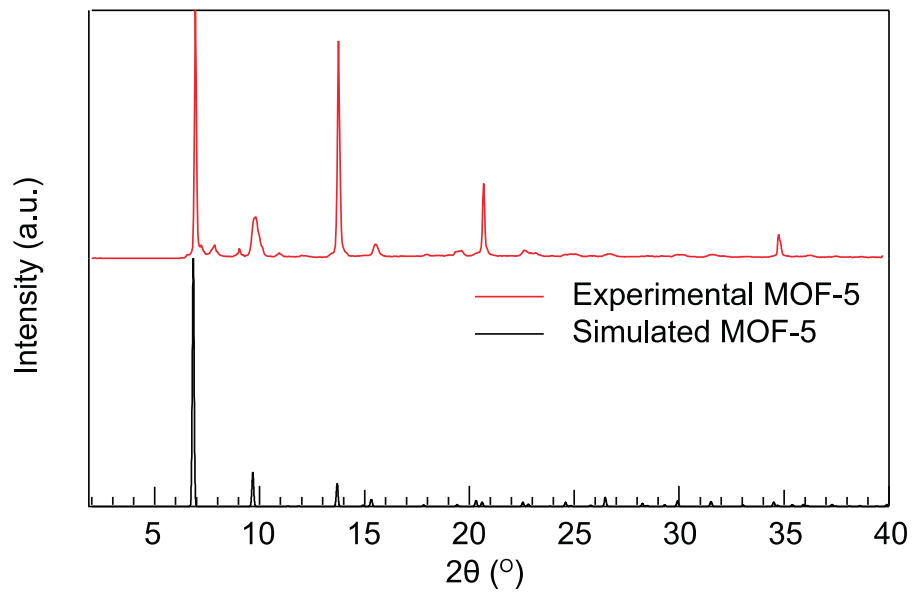

Figure S6: Experimental and simulated powder X-ray diffraction patterns of MOF-5.

MIL-125. Following a procedure adapted from Dan-Hardi et. al, ${ }^{6}$ titanium (IV) ethoxide (0.3475 g, $1.52 \mathrm{mmol}$ ) was dissolved in DMF $(4.5 \mathrm{~mL})$ under inert atmosphere and added to a Teflon Parr bomb with terephthalic acid $(0.3794 \mathrm{~g}, 2.28 \mathrm{mmol})$ and methanol $(0.5 \mathrm{~mL})$. This mixture was then heated at $150^{\circ} \mathrm{C}$ for 16 hours. The resulting white solid was collected by centrifugation and washed with DMF ( $3 \times 15 \mathrm{~mL})$, methanol $(3 \times 15 \mathrm{~mL})$, and with hexane $(2 \times 15 \mathrm{~mL})$ each for 1 day over the course of 3 days. The solvent was decanted, and the product dried under vacuum at room temperature.

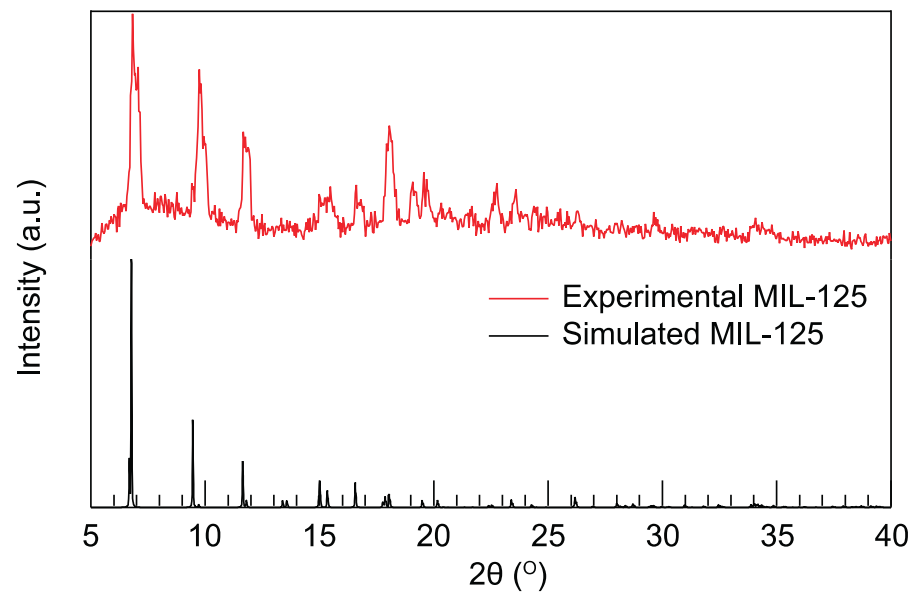

Figure S7: Experimental and simulated powder X-ray diffraction patterns of MIL-125. 
UiO-66. Following a procedure adapted from Katz et. al, ${ }^{7}$ zirconium (IV) chloride $(0.1262 \mathrm{~g}, 0.54$ $\mathrm{mmol})$ was dissolved in DMF (5 mL) in a $20-\mathrm{mL}$ scintillation vial under inert atmosphere. Upon exposing the vial to atmospheric conditions, concentrated hydrochloric acid $(1 \mathrm{~mL})$ was added, and the mixture was sonicated for 20 minutes and transferred to 100-mL Schott jar with a Teflon ring. Terephthalic acid $(0.1232 \mathrm{~g}, 0.74 \mathrm{mmol})$ and DMF $(10 \mathrm{~mL})$ were added to the same jar and sonicated for additional 20 minutes. After contents fully dissolved, the jar was placed in a convection oven at $80^{\circ} \mathrm{C}$ overnight (12 hours). The resulting white powder was washed with DMF $(2 \times 30 \mathrm{~mL})$ and ethanol $(2 \times 30 \mathrm{~mL})$ over the course of 2 days and dried under vacuum at room temperature.

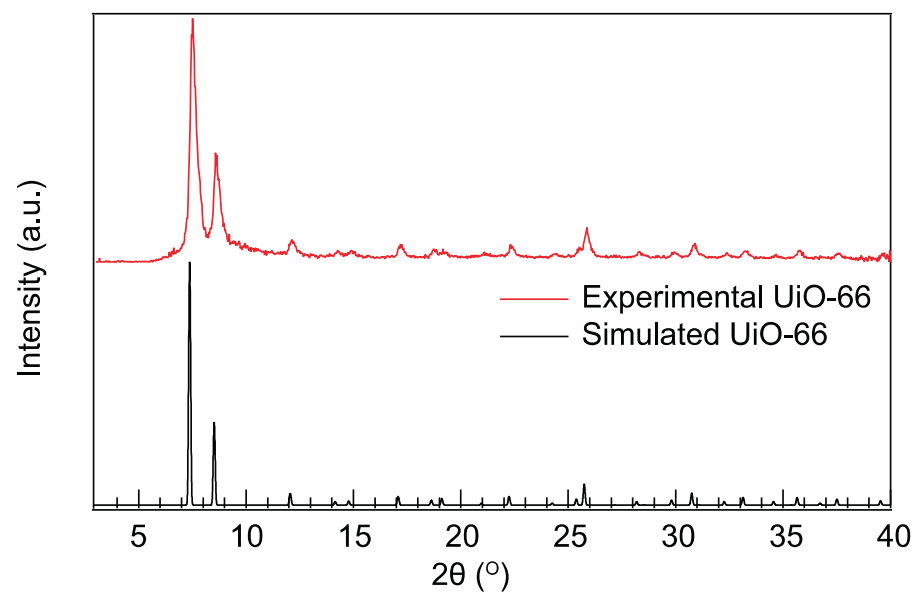

Figure S8: Experimental and simulated powder X-ray diffraction patterns of UiO-66.

MIL-125- $\mathrm{NH}_{2}$. Following a procedure adapted from Dan-Hardi et. al, ${ }^{6}$ titanium (IV) isopropoxide $(0.2851 \mathrm{~g}, 1.00 \mathrm{mmol})$ was dissolved in DMF $(4.0 \mathrm{~mL})$ under inert atmosphere and added to a Teflon Parr bomb with 2-aminoterephthalic acid $(0.5233 \mathrm{~g}, 3.0 \mathrm{mmol})$ and methanol $(1 \mathrm{~mL})$. The mixture was then heated at $150{ }^{\circ} \mathrm{C}$ for 15 hours. The resulting yellow powder was collected by centrifugation and washed with DMF ( $3 \times 6 \mathrm{~mL})$ and with methanol (3 x $6 \mathrm{~mL}$ each), each for 1 day over the course of 2 days. The solvent was decanted, and the product was dried under dynamic vacuum on a Schlenk line overnight at room temperature.



Figure S9: Experimental and simulated powder X-ray diffraction patterns of MIL-125-NH2. 
$C u B D C$. Following procedures adapted from Rodenas et. al, ${ }^{8}$ copper (II) nitrate trihydrate (1.0534 $\mathrm{g}, 4.36 \mathrm{mmol})$, terephthalic acid $(0.7242 \mathrm{~g}, 4.36 \mathrm{mmol})$, and DMF (87 mL) were combined in 250 $\mathrm{mL}$ RB flask fitted with a reflux condenser, and heated at $100^{\circ} \mathrm{C}$ for 24 hours. The resulting blue powder was collected by centrifugation and washed with DMF $(3 \times 15 \mathrm{~mL})$ and DCM (3 x 15 $\mathrm{mL}$ ) to remove remaining linker equivalents each for 1 day over the course of 2 days. The product was stored in DCM and before use was dried in a vacuum oven overnight at room temperature.

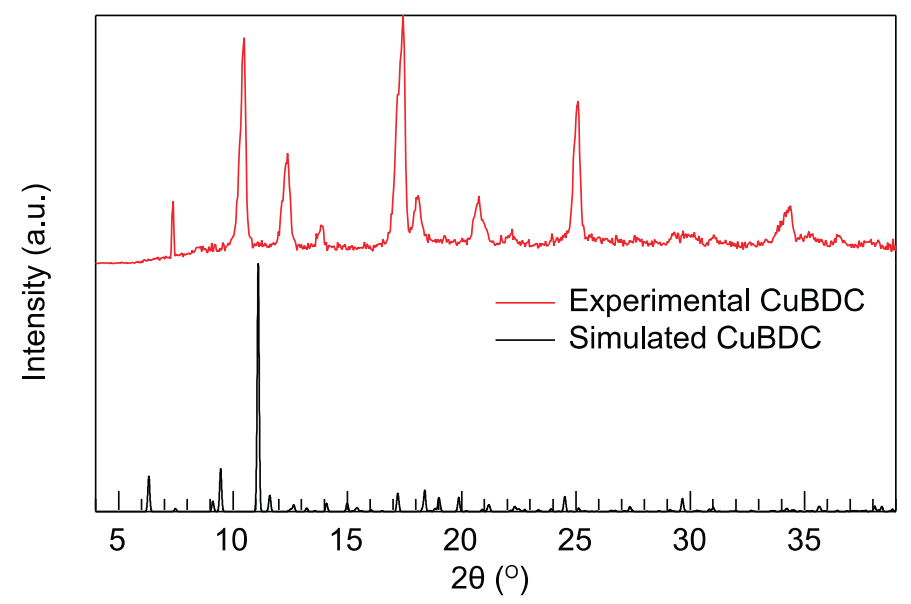

Figure S10: Experimental and simulated powder X-ray diffraction patterns of CuBDC. 
Cu-MOF-74. Following a procedure adapted from Calleja et. al, ${ }^{9}$ copper (II) nitrate trihydrate (0.5949 g, $2.46 \mathrm{mmol}), 2,5$-dihydroxyterephthalic acid (0.2222g, $1.12 \mathrm{mmol})$, DMF (23.8 $\mathrm{mL})$ and isopropanol $(1.2 \mathrm{~mL})$ were combined in $100-\mathrm{mL}$ Schott jar with a Teflon ring and sonicated until the solids dissolved. The mixture was then heated in a convection oven for 18 hours at $80{ }^{\circ} \mathrm{C}$. The resulting black solid was washed and soaked in DMF $(3 \times 15 \mathrm{~mL})$ and methanol $(3 \times 15 \mathrm{~mL})$ each for 1 day over the course of 2 days and dried in a vacuum oven at room temperature.

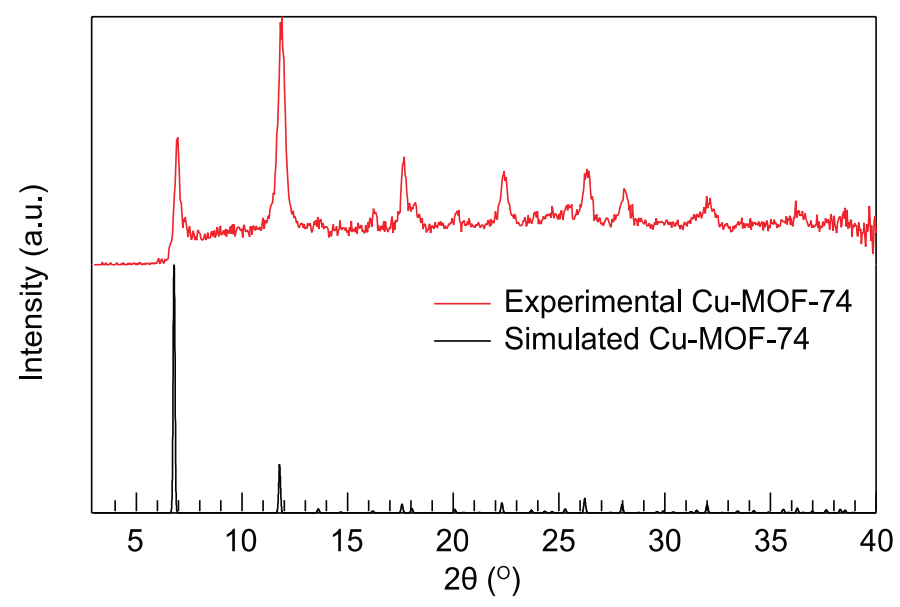

Figure S11: Experimental and simulated powder X-ray diffraction patterns of Cu-MOF-74. 
DRIFTS Section I: spectra with assigned carboxylate stretches

1,3,5-benzenetricarboxylic acid (BTC)

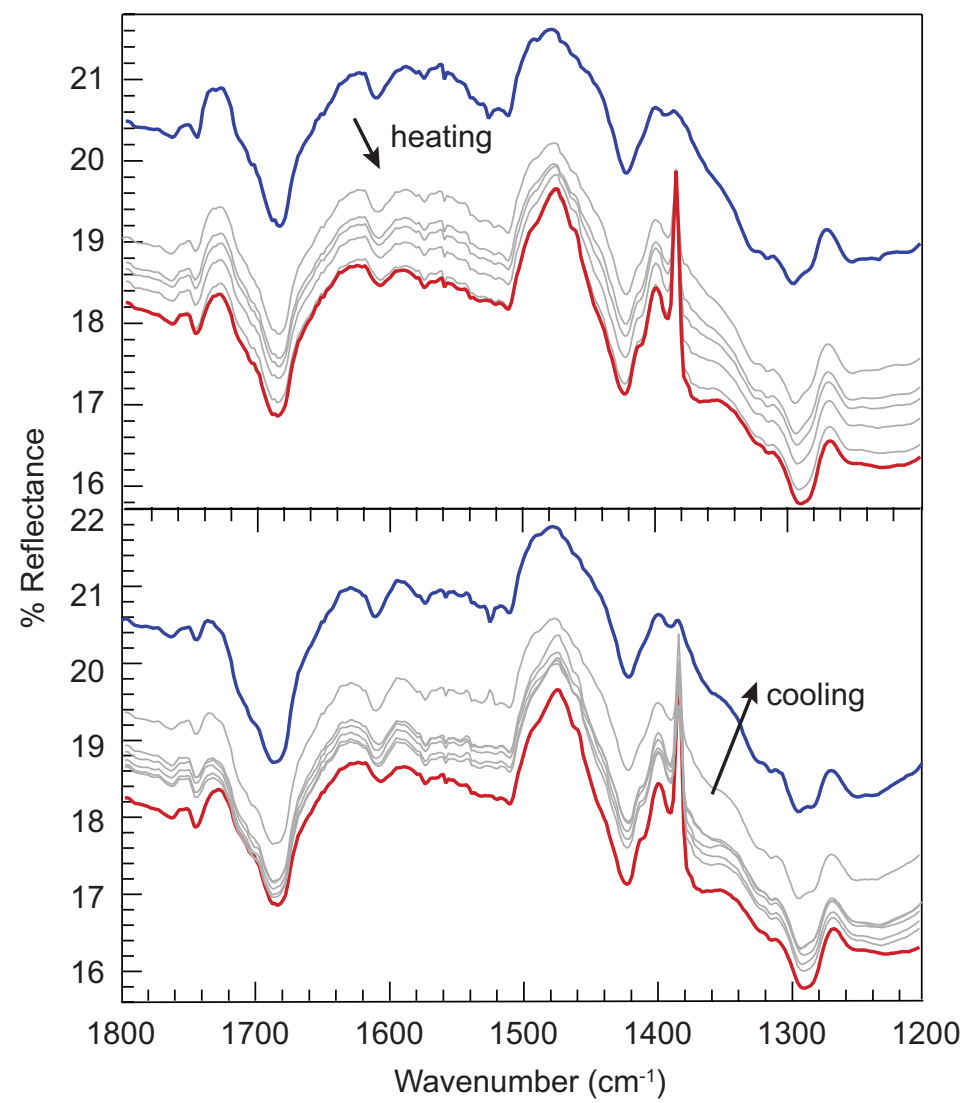

Figure S12: Variable-temperature diffuse reflectance infrared Fourier transform spectra (VTDRIFTS) of BTC between $200^{\circ} \mathrm{C}$ (red) and room temperature (blue). 
Sodium benzoate



Figure S13: VT-DRIFTS of sodium benzoate between $200{ }^{\circ} \mathrm{C}$ (red) and $-100{ }^{\circ} \mathrm{C}$ (blue). The asymmetric carboxylate stretch is observed at $1553.1 \mathrm{~cm}^{-1}$ and the symmetric is observed at 1420.0 $\mathrm{cm}^{-1}$. Interestingly, all bands in the $1800-1200 \mathrm{~cm}^{-1}$ range appear to redshift and broaden.


Figure S14: Normalized Spectra of Carboxylates. (A) Normalized symmetric (top) and asymmetric (bottom) carboxylate stretches shift respectively by 6.8 and 4.8 wavenumbers to lower in energy with higher temperature. Red trace was collected at $200{ }^{\circ} \mathrm{C}$ and the dark blue was collected at $-100^{\circ} \mathrm{C}$. (B) Scatter plots show peak positions as a function of temperature. Hollow circles represent average values of experimental values; error bars represent different cycles. 


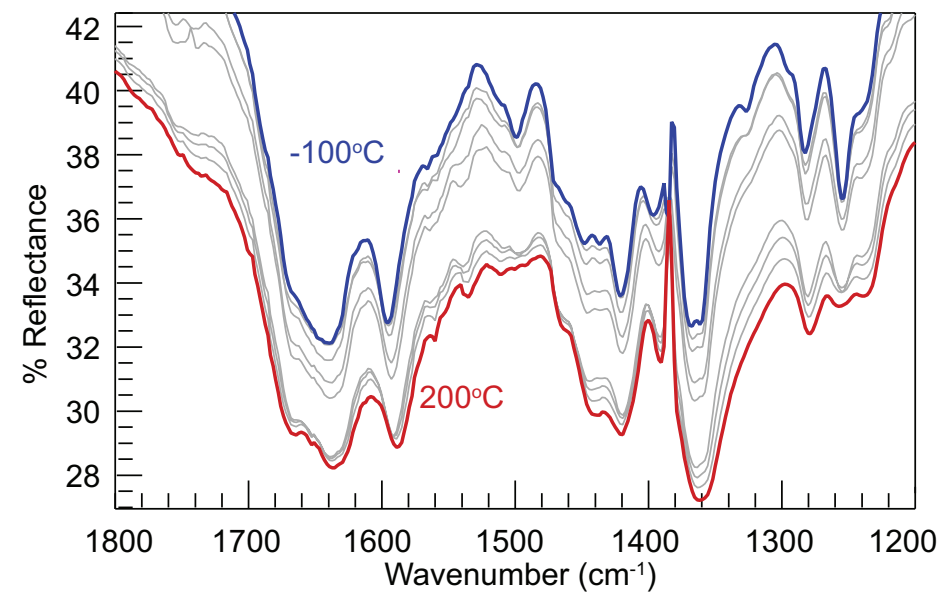

Figure S15: Unnormalized VT-DRIFTS of HKUST-1. The asymmetric carboxylate stretch $\left(v_{a}\right)$ is observed at $1592.9 \mathrm{~cm}^{-1}$ and the symmetric $\left(v_{S}\right)$ is observed at $1365.7 \mathrm{~cm}^{-1}$.
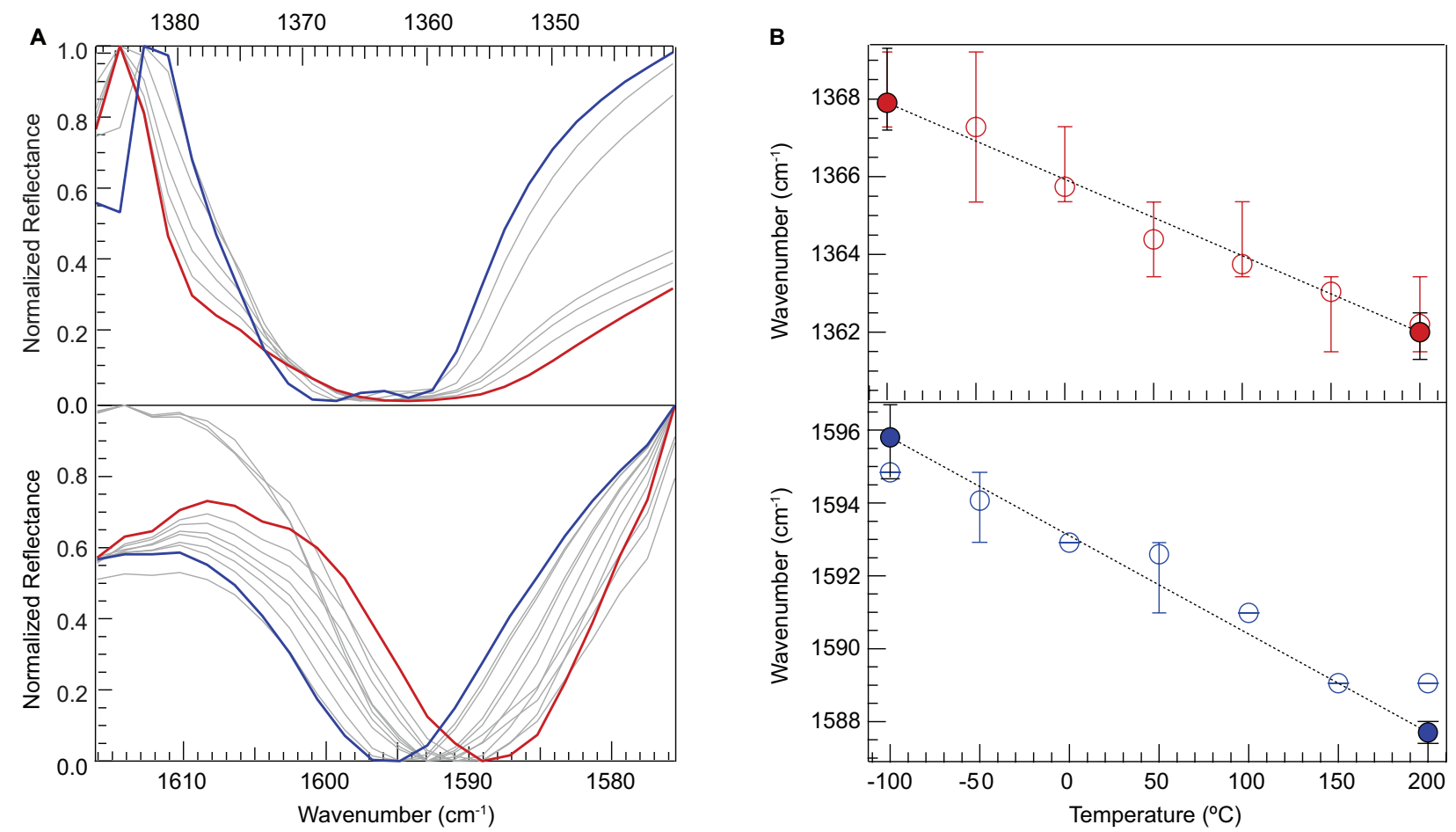

Figure S16: (A) Normalized spectra of HKUST-1 symmetric (top panel) and asymmetric (bottom) carboxylate stretches between $200{ }^{\circ} \mathrm{C}$ (red) and $-100{ }^{\circ} \mathrm{C}$ (blue). Both symmetric and asymmetric experimentally redshift by 5.8 wavenumbers with higher temperature. (B) Scatterplots of peak position versus temperature for symmetric (top panel) and asymmetric (bottom). Hollow circles indicate average value of experimental data points, filled circles are Gaussian fitted values. Error bars include values for 3 cycles. 


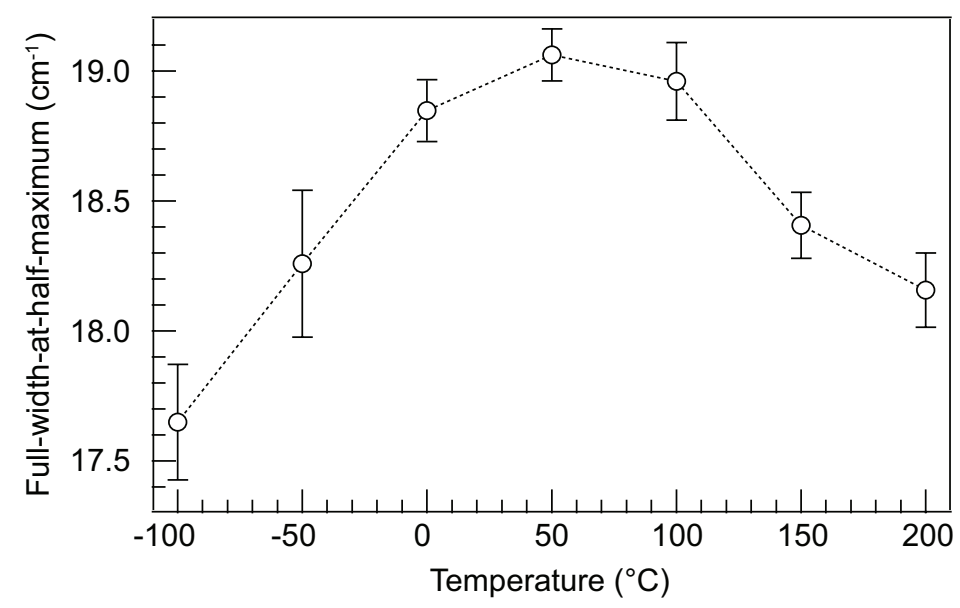

Figure S17: Full-width-at-half maximum values versus temperature for the asymmetric carboxylate stretch of HKUST-1.

$M U V-10(\mathrm{Ca})$

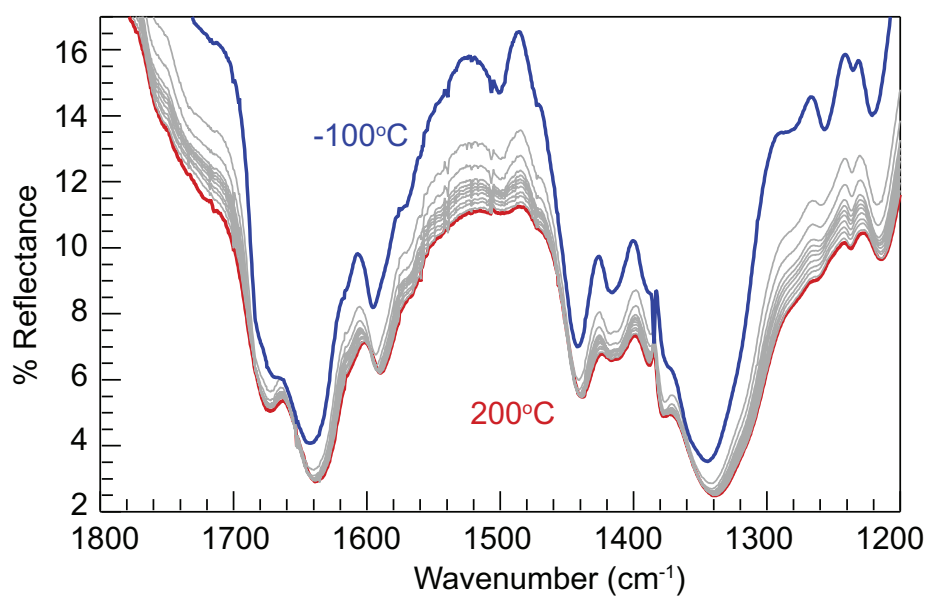

Figure S18: Unnormalized VT-DRIFTS of MUV-10 (Ca). Data were collected with $0.4821 \mathrm{~cm}^{-}$ ${ }^{1}$ resolution. The asymmetric carboxylate stretch is observed at $1641.4 \mathrm{~cm}^{-1}$ and the symmetric is observed at $1342.7 \mathrm{~cm}^{-1}$. 

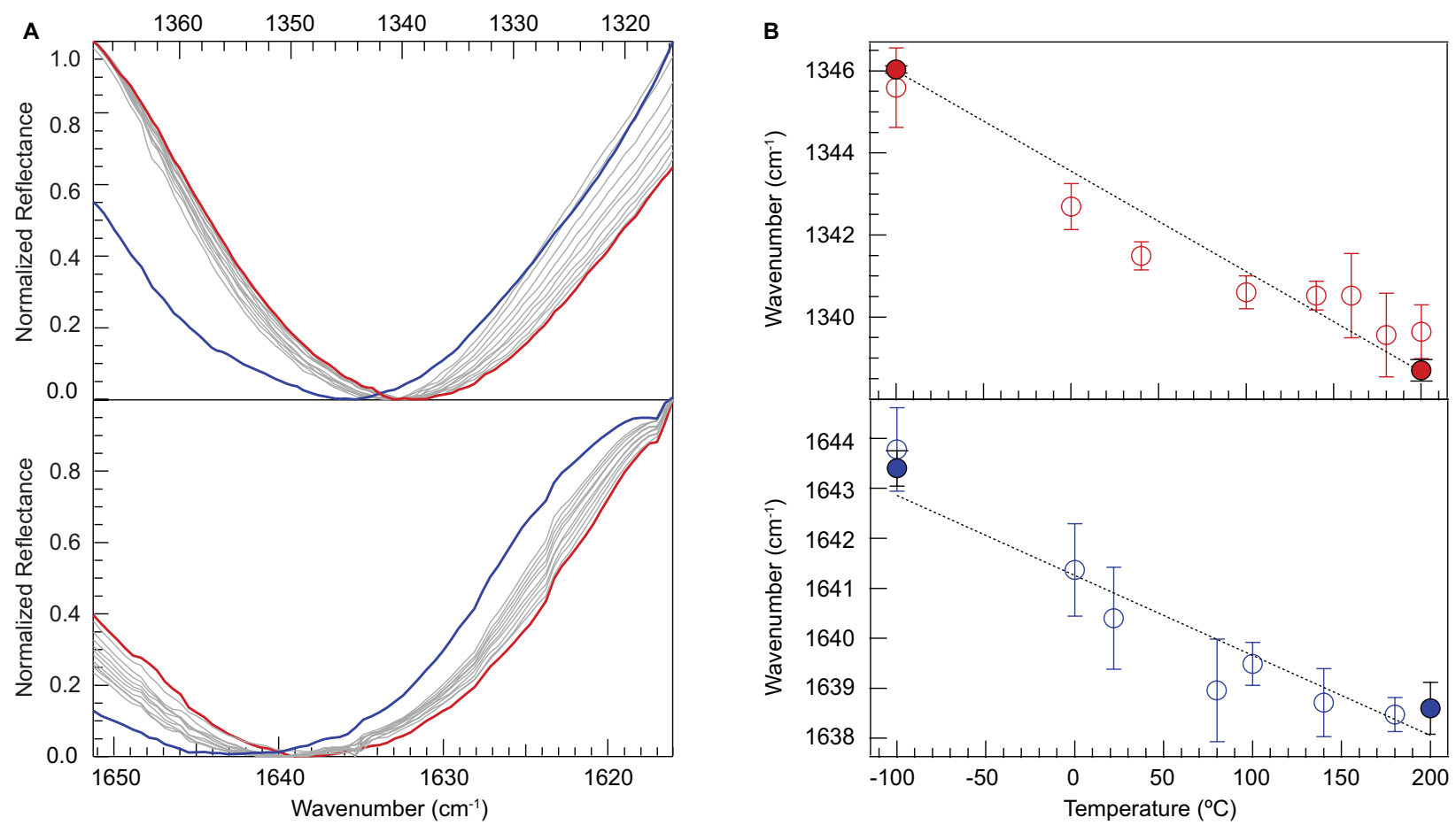

Figure S19: Normalized VT-DRIFTS of carboxylates stretches for MUV-10 (Ca). (A): Over the course of 300 degrees, symmetric (top panel) and asymmetric (bottom) stretches experimentally redshift by 6.0 and 5.3 wavenumbers respectively with higher temperature. (B) Scatterplots of shifts in symmetric (top panel) and asymmetric (bottom) stretches between $200{ }^{\circ} \mathrm{C}$ and $-100{ }^{\circ} \mathrm{C}$. Hollow circles indicate experimental data points, filled circles are Gaussian fitted values. Error bars represent standard deviations from 3 cycles. 




Figure S20: Unnormalized VT-DRIFTS of MUV-10 (Mn). Data collected with $0.4821 \mathrm{~cm}^{-1}$ resolution. The asymmetric carboxylate stretch is observed at $1628.1 \mathrm{~cm}^{-1}$ and the symmetric is observed at $1328.6 \mathrm{~cm}^{-1}$.
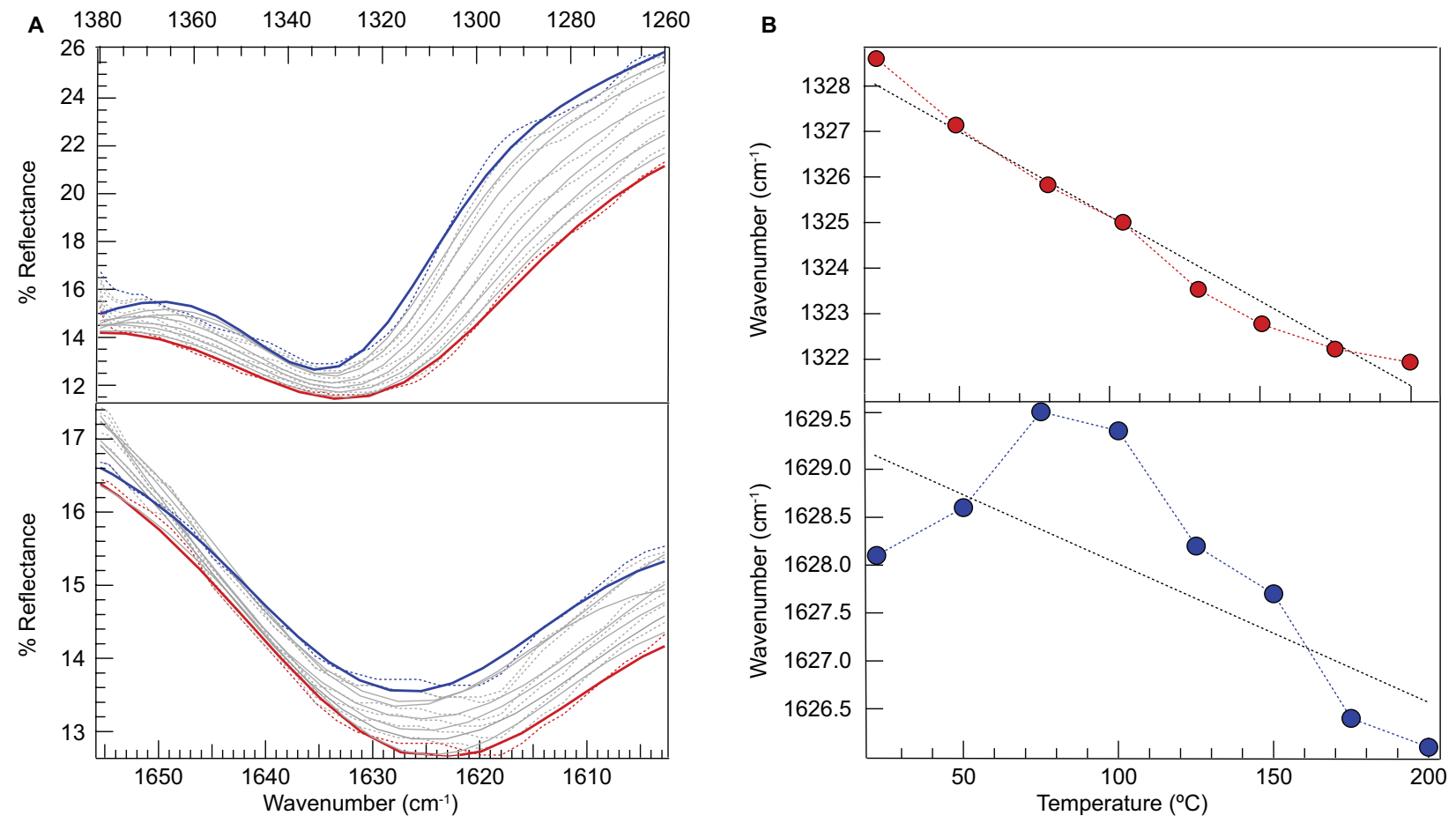

Figure S21: VT-DRIFTS of MUV-10 (Mn) carboxylate stretches fitted to Gaussian functions. (A): Between $200{ }^{\circ} \mathrm{C}$ and $-100{ }^{\circ} \mathrm{C}$, the symmetric (top panel) and asymmetric (bottom) stretches experimentally redshift by 7.1 and 2.0 wavenumbers at higher temperature. (B) Scatterplots of peak positions for the symmetric (top panel) and asymmetric (bottom) stretches. Filled circles are Gaussian-fitted values. 


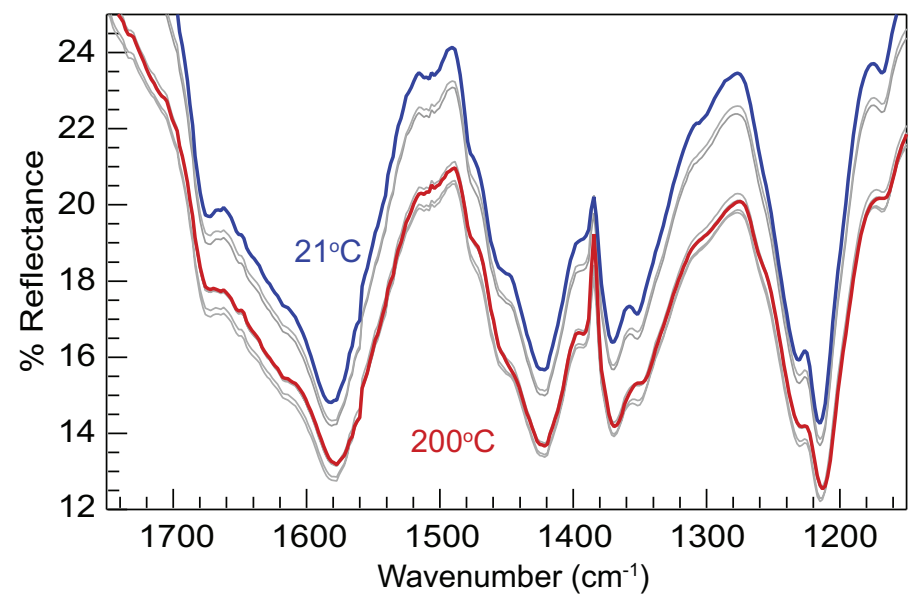

Figure S22: VT-DRIFTS of Mg-MOF-74. The asymmetric stretch is observed at $1581.3 \mathrm{~cm}^{-1}$ and the symmetric is observed at $1371.1 \mathrm{~cm}^{-1}$.
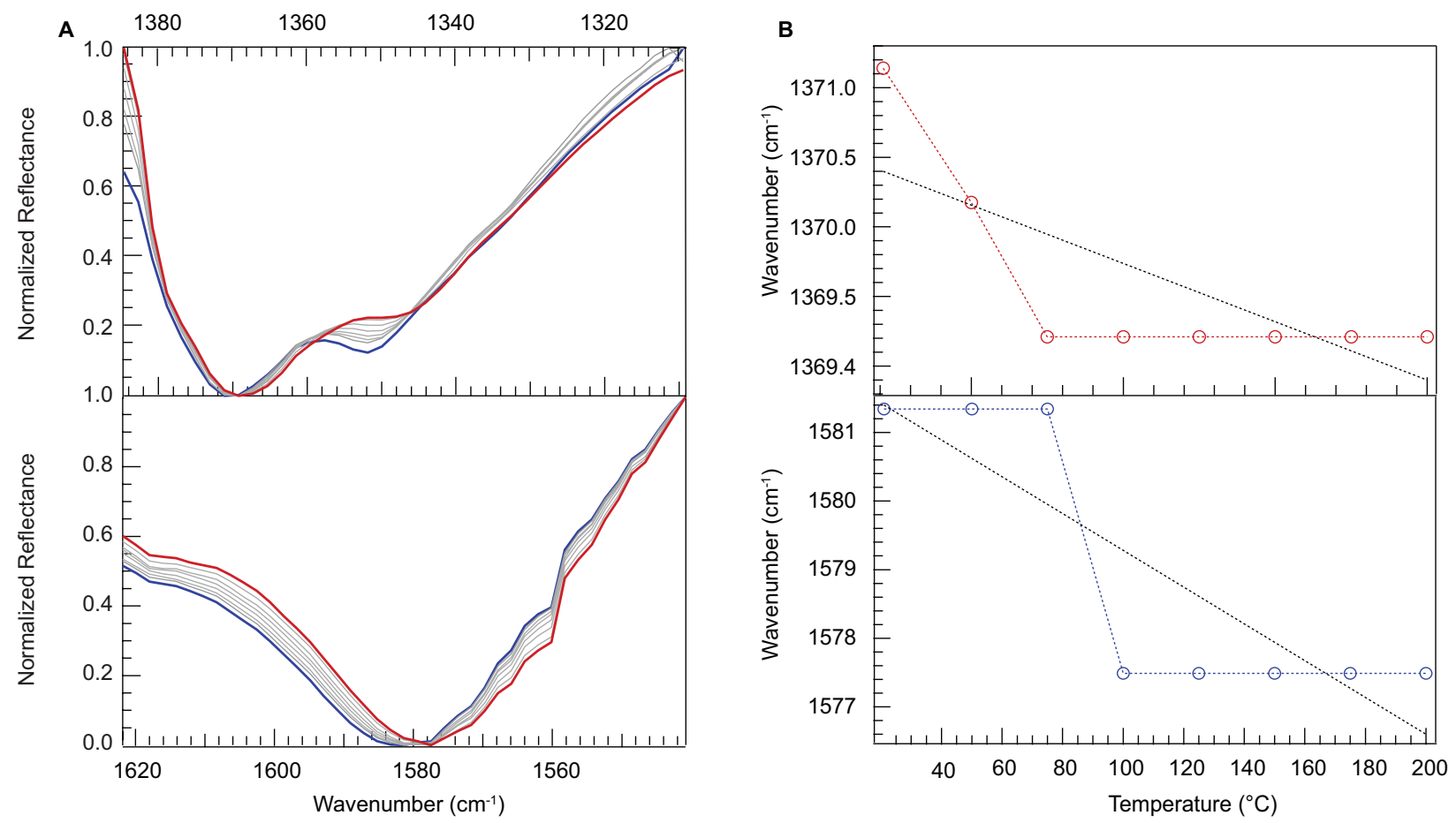

Figure S23: Normalized VT-DRFTS of Mg-MOF-74 carboxylates. (A): Over the course of 178 degrees, symmetric (top panel) and asymmetric (bottom) stretches experimentally redshift by 1.9 and 3.5 wavenumbers respectively at higher temperatures. (B) The scatterplots represent overall shift in the symmetric (top panel) and asymmetric (bottom) stretches over the course of 178 degrees. Hollow circles indicate experimental data points for one cycle. 


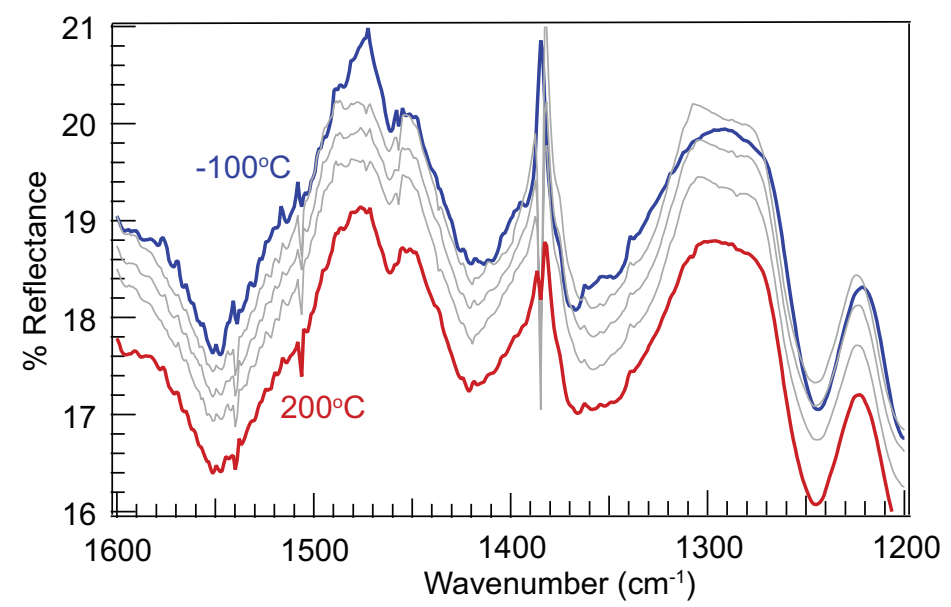

Figure S24: VT-DRIFTS of Zn-MOF-74. The asymmetric carboxylate stretch is observed at $1549.5 \mathrm{~cm}^{-1}$ and the symmetric is observed at $1357.3 \mathrm{~cm}^{-1}$.
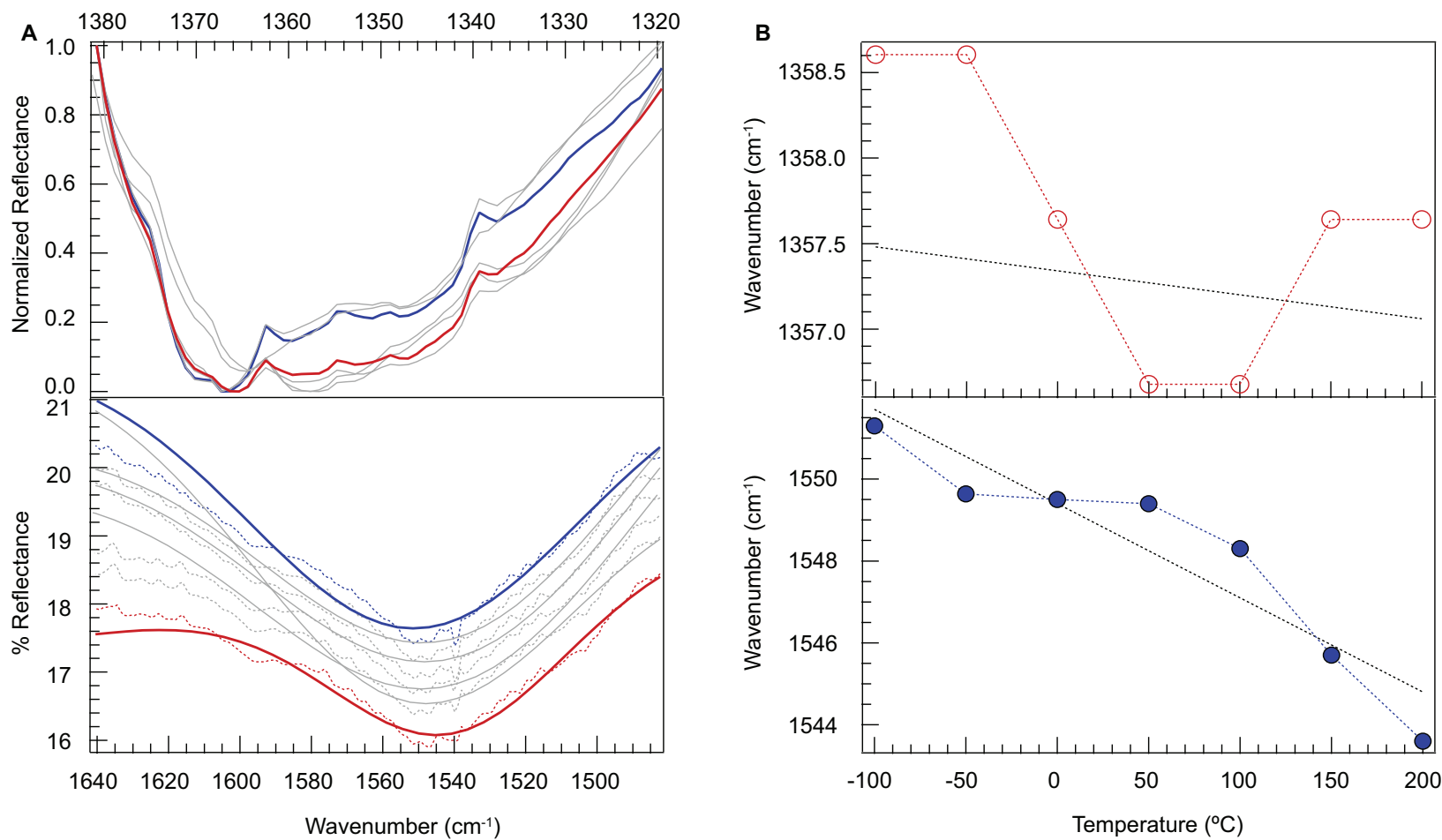

Figure S25: Normalized VT-DRFITS of Zn-MOF-74 carboxylate stretches with Gaussian fits. (A): Over the course of 300 degrees, the symmetric (top panel) and asymmetric (bottom) stretches shift by 1.0 and $7.7 \mathrm{~cm}^{-1}$ respectively at higher temperatures. (B) Scatterplots show peak positions of the symmetric (top panel) and asymmetric (bottom) stretches versus temperature. Hollow circles indicate experimental data points for one cycle; filled circles represent values from Gaussian fits. 
$M O F-5$

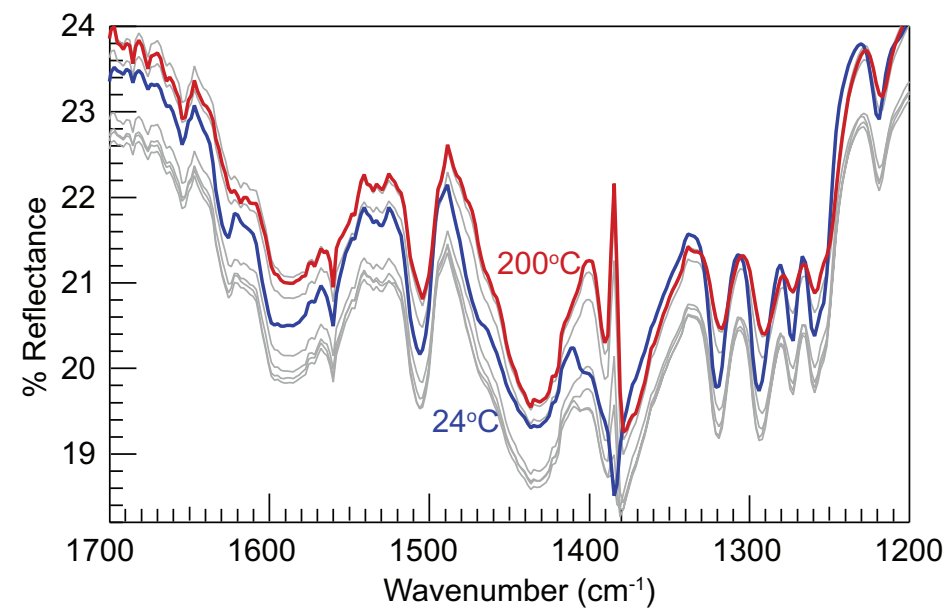

Figure S26: VT-DRIFTS of MOF-5. The asymmetric carboxylate stretch is observed at 1590.4 $\mathrm{cm}^{-1}$ and the symmetric is observed at $1435.5 \mathrm{~cm}^{-1}$.
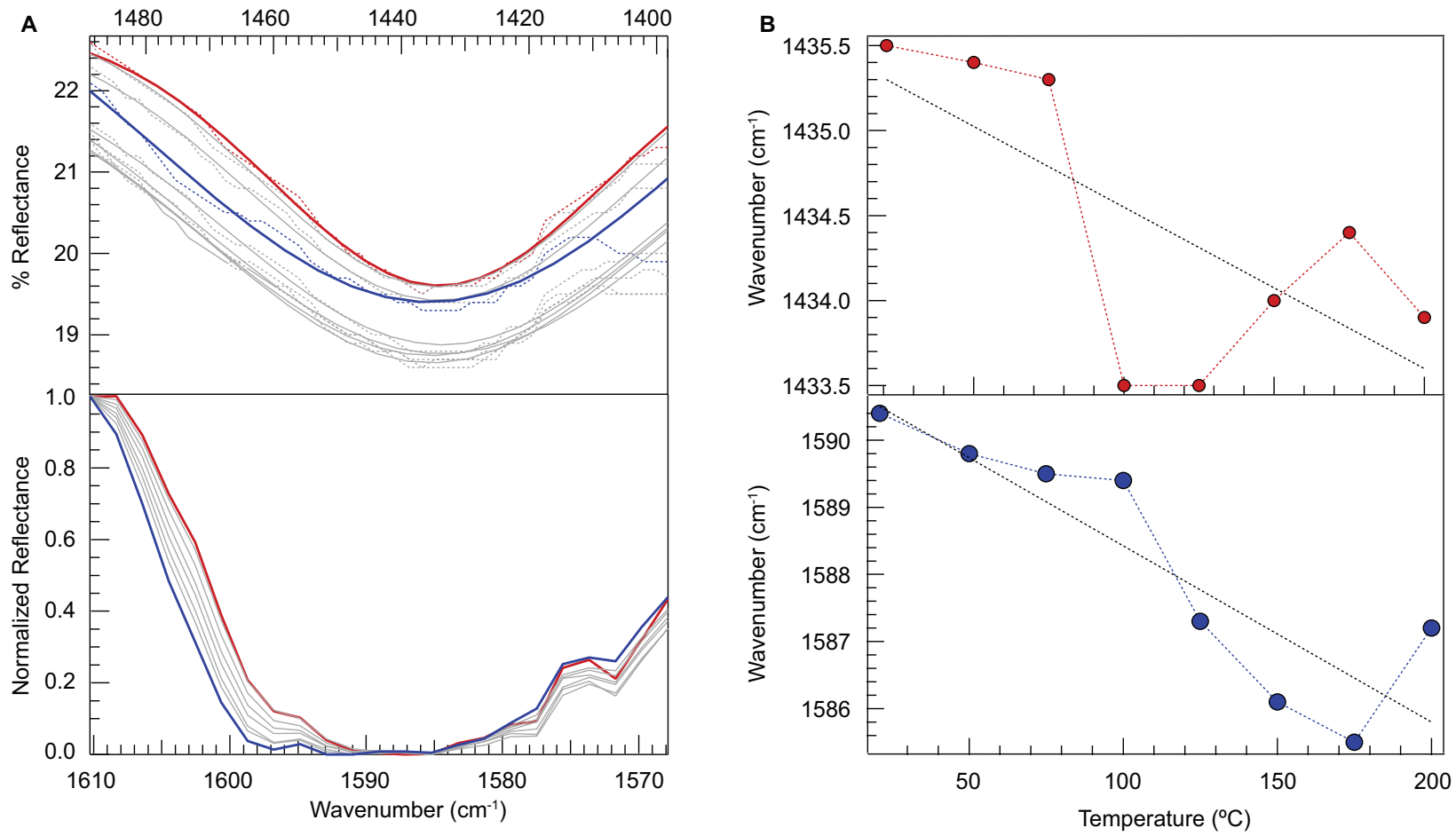

Figure S27: VT-DRFITS of MOF-5 carboxylate stretches with Gaussian fits. (A): Over a temperature range of 179 degrees, the symmetric (top panel) and asymmetric (bottom) stretches redshift by 1.6 and $3.2 \mathrm{~cm}^{-1}$ with increased temperature. (B) Scatterplots show peak positions of symmetric (top panel) and asymmetric (bottom) stretches versus temperature. Hollow circles indicate experimental data points for one cycle and filled circles represent values from Gaussian fitting. 
MIL-125

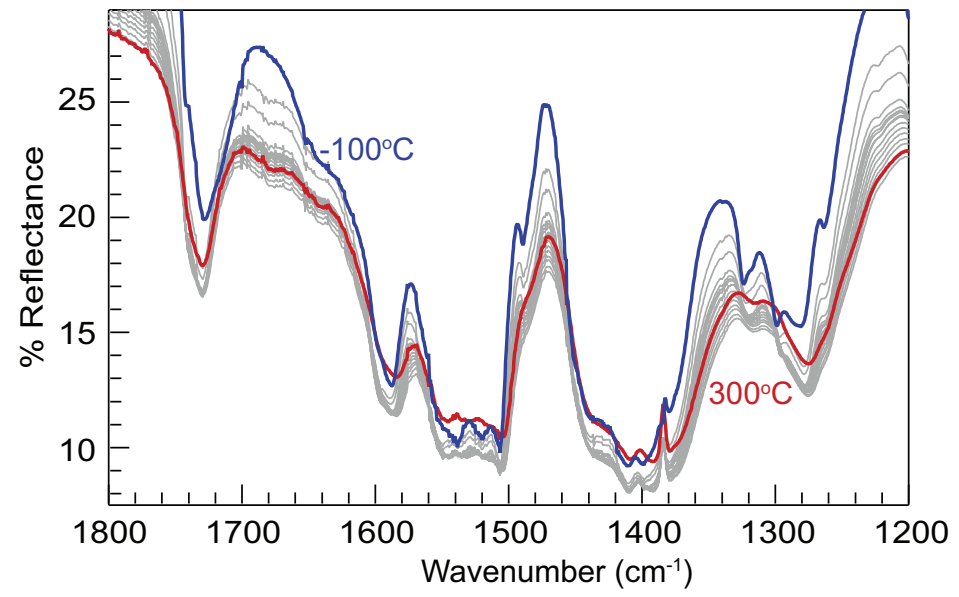

Figure S28: Unnormalized VT-DRIFTS of MIL-125. The symmetric carboxylate stretch appears at $1398.6 \mathrm{~cm}^{-1}$ and the asymmetric at $1597.7 \mathrm{~cm}^{-1}$.
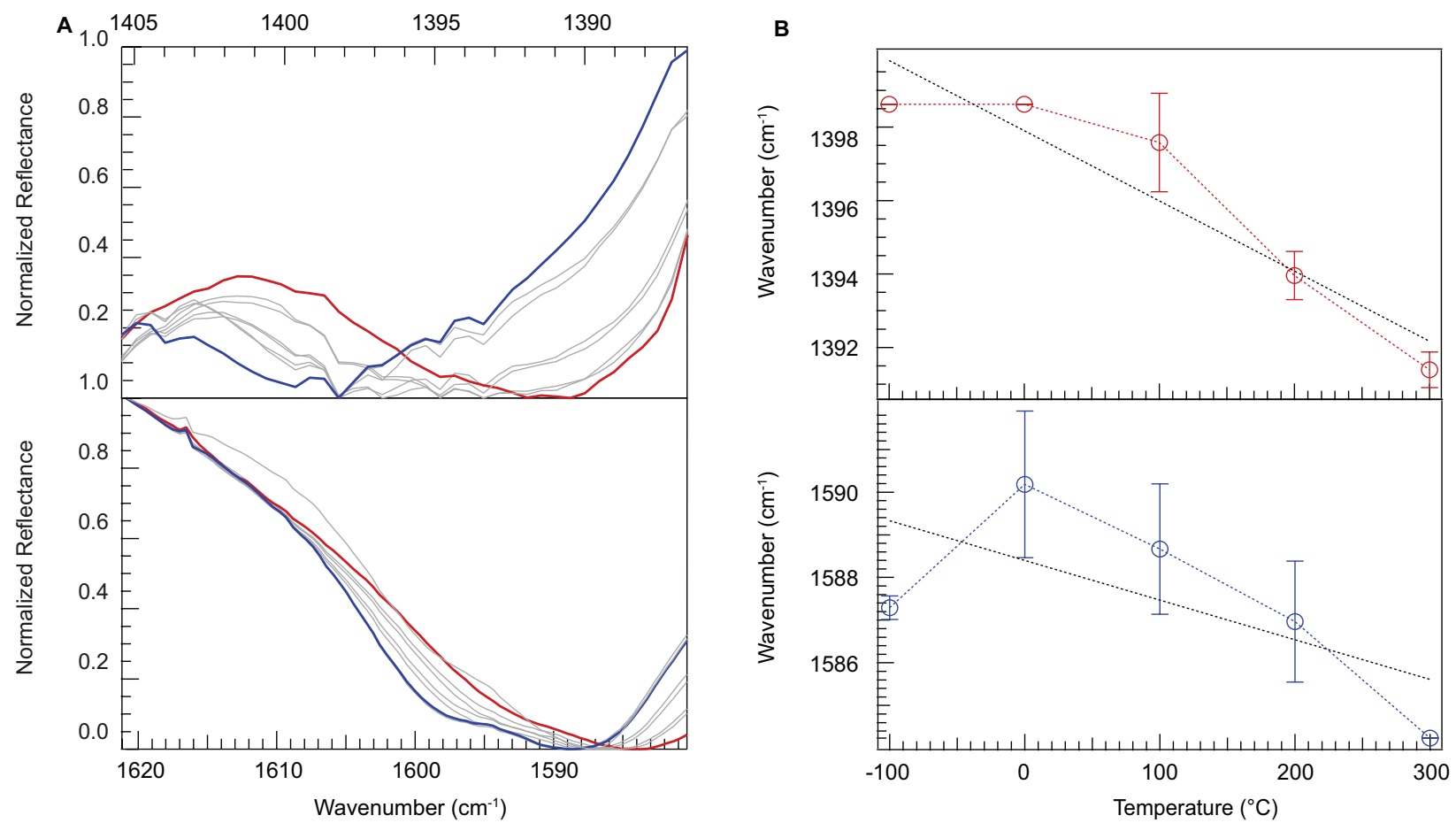

Figure S29: Normalized VT-DRIFTS of carboxylate stretches. (A): Over a temperature range of $400{ }^{\circ} \mathrm{C}$, the symmetric (top panel) and asymmetric (bottom) stretches redshift by 7.2 and 3.0 $\mathrm{cm}^{-1}$ with increased temperature. (B) Scatterplot of peak positions of symmetric (top panel) and asymmetric (bottom) stretches versus temperature. Hollow circles indicate average values of experimental data points for one cycle and error bars represent different cycles. 




Figure S30: Unnormalized VT-DRIFTS of UiO-66. The symmetric carboxylate stretch appears at $1398.1 \mathrm{~cm}^{-1}$ and the asymmetric at $1587.1 \mathrm{~cm}^{-1}$.
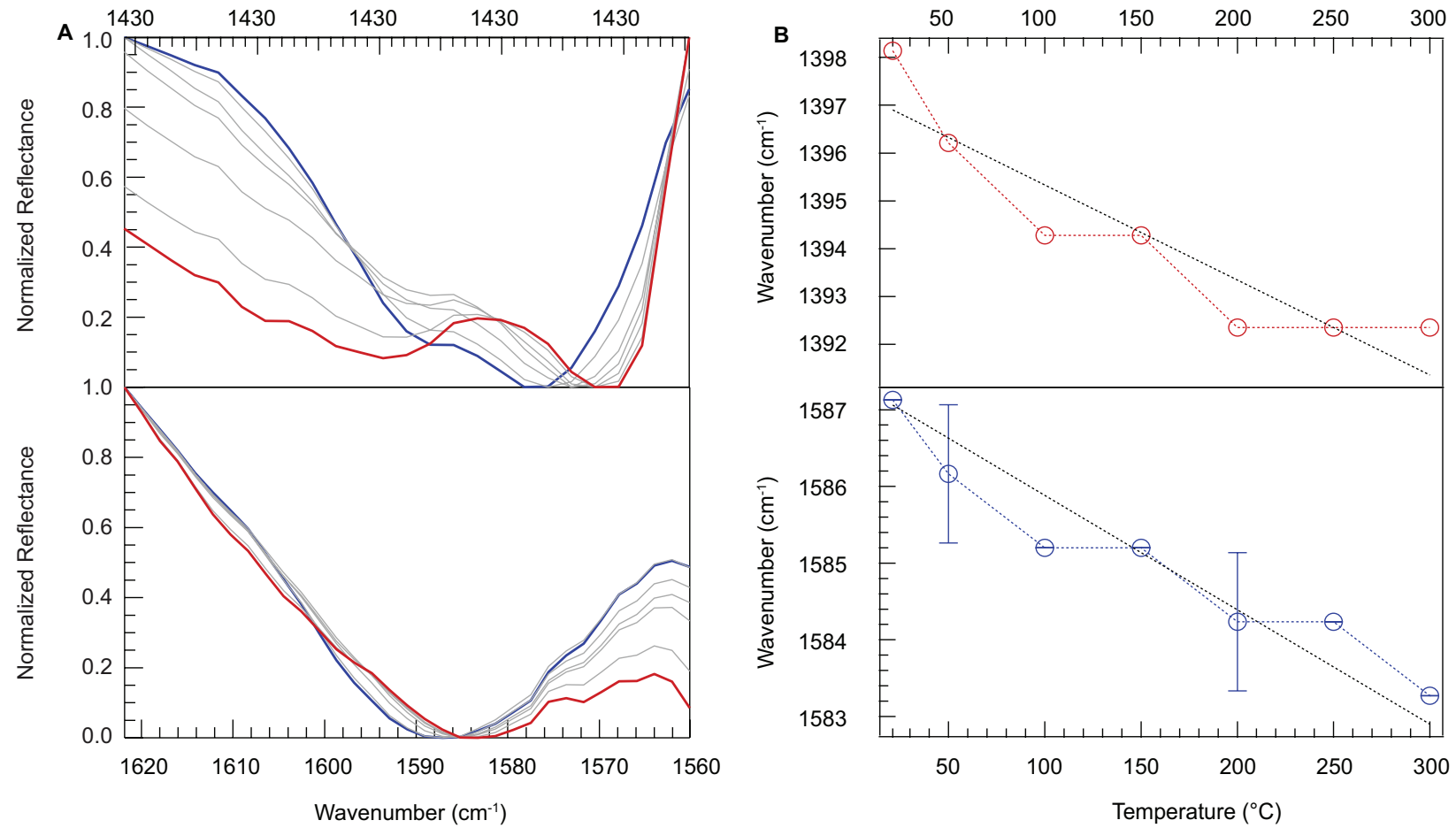

Figure S31: Normalized VT-DRIFTS of UiO-66 carboxylate stretches. (A) Over a temperature range spanning 400 degrees, symmetric stretch (top panel) redshifts by $5.8 \mathrm{~cm}^{-1}$ with increased temperature. No further shifting was observed upon above $200{ }^{\circ} \mathrm{C}$. Over this temperature range, the asymmetric stretch (bottom) redshifts by $3.9 \mathrm{~cm}^{-1}$ with increased temperature. (B) Scatterplot of peak positions of the symmetric (top panel) and asymmetric (bottom) stretches versus temperature. Hollow circles indicate average experimental data points and error bars were calculated from variance in additional cycles. 
MIL-125- $\mathrm{NH}_{2}$

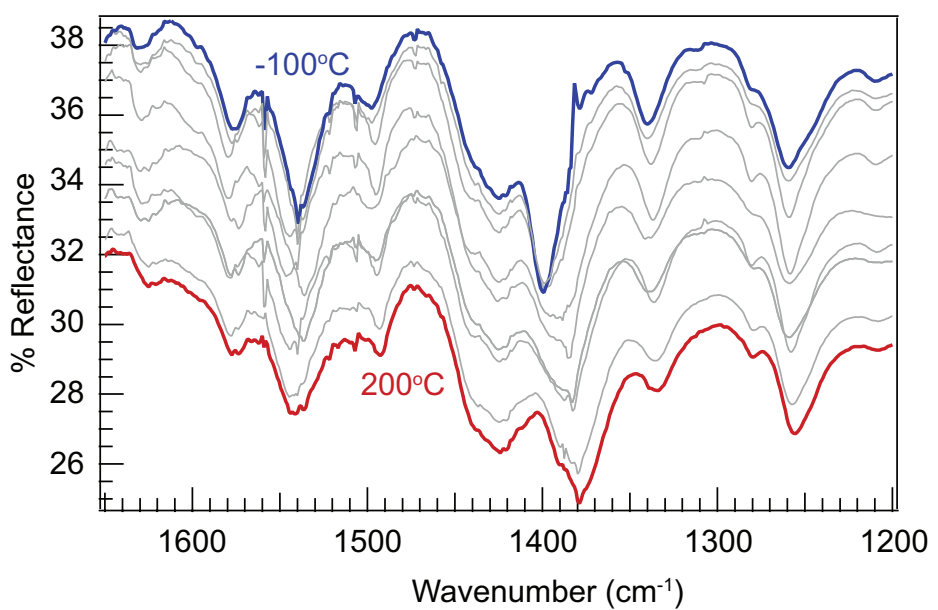

Figure S32: Unnormalized VT-DRIFTS of MIL-125- $\mathbf{N H}_{2}$. The symmetric carboxylate stretch appears at $1387.9 \mathrm{~cm}^{-1}$, and asymmetric at $1575.8 \mathrm{~cm}^{-1}$.
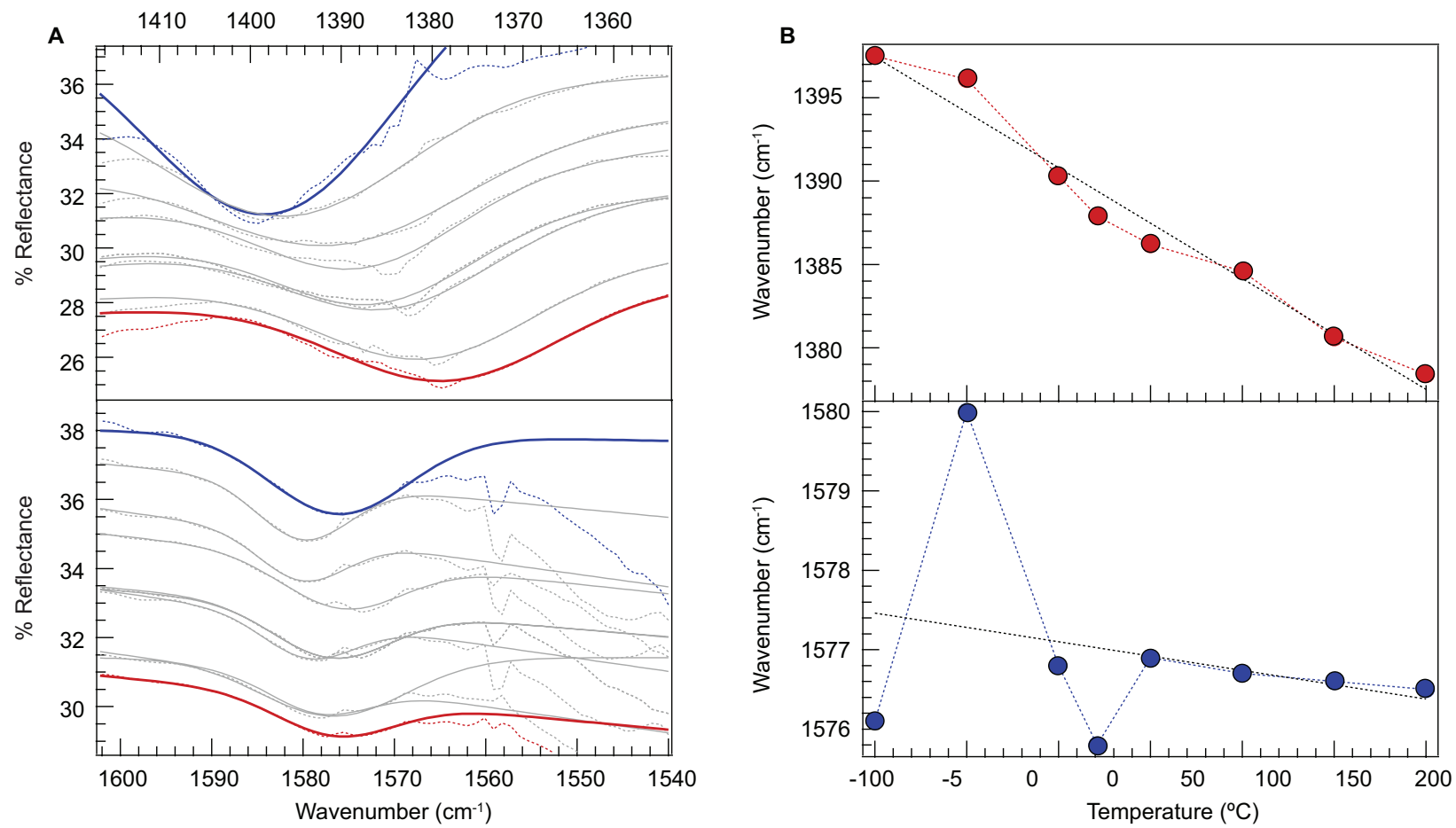

Figure S33: VT-DRIFTS of MIL-125- $\mathrm{NH}_{2}$ carboxylate stretches with Gaussian fits. (A): Over a temperature range spanning 300 degrees, the symmetric (top panel) and asymmetric (bottom) stretches redshift by $19.1 \mathrm{~cm}^{-1}$ and blueshift by $1.1 \mathrm{~cm}^{-1}$ with increased temperature, respectively. (B) Scatterplots of the peak positions of the symmetric (top) and asymmetric (bottom) stretches versus temperature. Filled circles represent values from Gaussian fitting. 
Table S1 Summary of asymmetric $\left(v_{\mathrm{a}}\right)$ and symmetric $\left(v_{\mathrm{s}}\right)$ peak positions and redshifts of MOFs studied here through VT-DRIFTS under dynamic vacuum.

\begin{tabular}{|c|c|c|c|c|c|}
\hline MOF Name & $\mathrm{v}_{\mathrm{A}}\left(\mathrm{cm}^{-1}\right)$ & $\mathrm{V}_{\mathrm{S}}\left(\mathrm{cm}^{-1}\right)$ & $v_{A}$ shift $\left(\mathrm{cm}^{-1}\right)$ & vs shift $\left(\mathrm{cm}^{-1}\right)$ & $\Delta \mathrm{v}\left(\mathrm{cm}^{-1}\right)$ \\
\hline CuBTC & 1592.9 & 1365.7 & 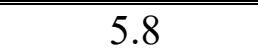 & 5.8 & 227.2 \\
\hline MUV-10 (Ca) & 1641.4 & 1342.7 & 5.3 & 6.0 & 298.7 \\
\hline MUV-10 (Mn) & 1628.1 & 1328.6 & 2.0 & 7.1 & 299.5 \\
\hline Mg-MOF-74 & 1581.3 & 1371.1 & 3.5 & 1.9 & 210.2 \\
\hline $\mathrm{Zn}-\mathrm{MOF}-74$ & 1549.5 & 1367.3 & 7.7 & 1.0 & 182.2 \\
\hline MOF-5 & 1590.4 & 1435.5 & 3.2 & 1.6 & 154.5 \\
\hline MIL-125 & 1597.7 & 1398.6 & 3.0 & 7.2 & 199.1 \\
\hline MIL-125-NH 2 & 1575.8 & 1387.9 & 1.1 & 19.1 & 187.9 \\
\hline $\mathrm{UiO}-66$ & 1587.1 & 1398.1 & 3.9 & 5.8 & 189.0 \\
\hline Sodium benzoate & 1553.1 & 1420.0 & 4.8 & 6.8 & 133.1 \\
\hline
\end{tabular}


DRIFTS Section II: Spectra with ambiguous peak assignments $C u B D C$

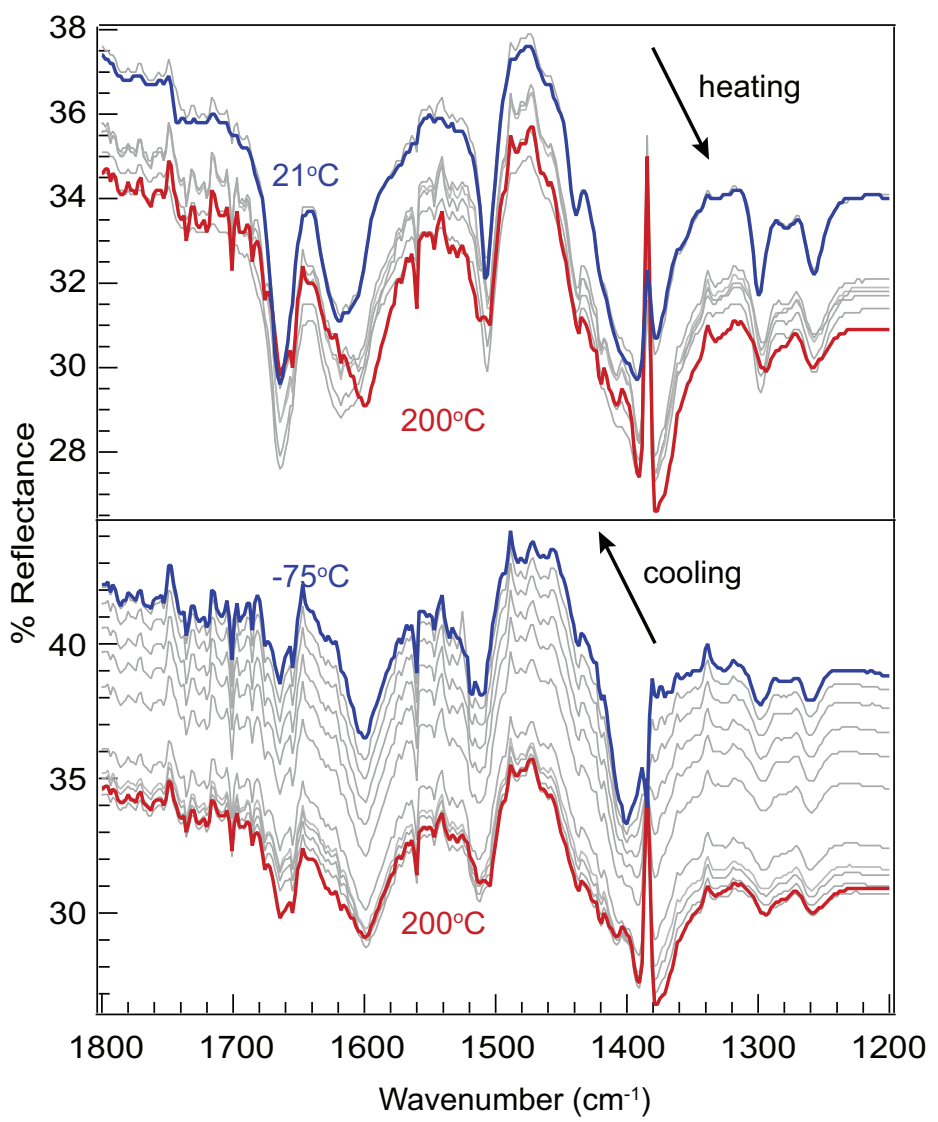

Figure S34: Unnormalized VT-DRIFTS of CuBDC. Because asymmetric stretch at $1620 \mathrm{~cm}^{-1}$ undergoes irreversible change and symmetric stretch at $1390 \mathrm{~cm}^{-1}$ is obliterated by the subtraction from software, it is difficult to interpret the minimum point in experimental data. 


\section{$\mathrm{Cu}-\mathrm{MOF}-74$}

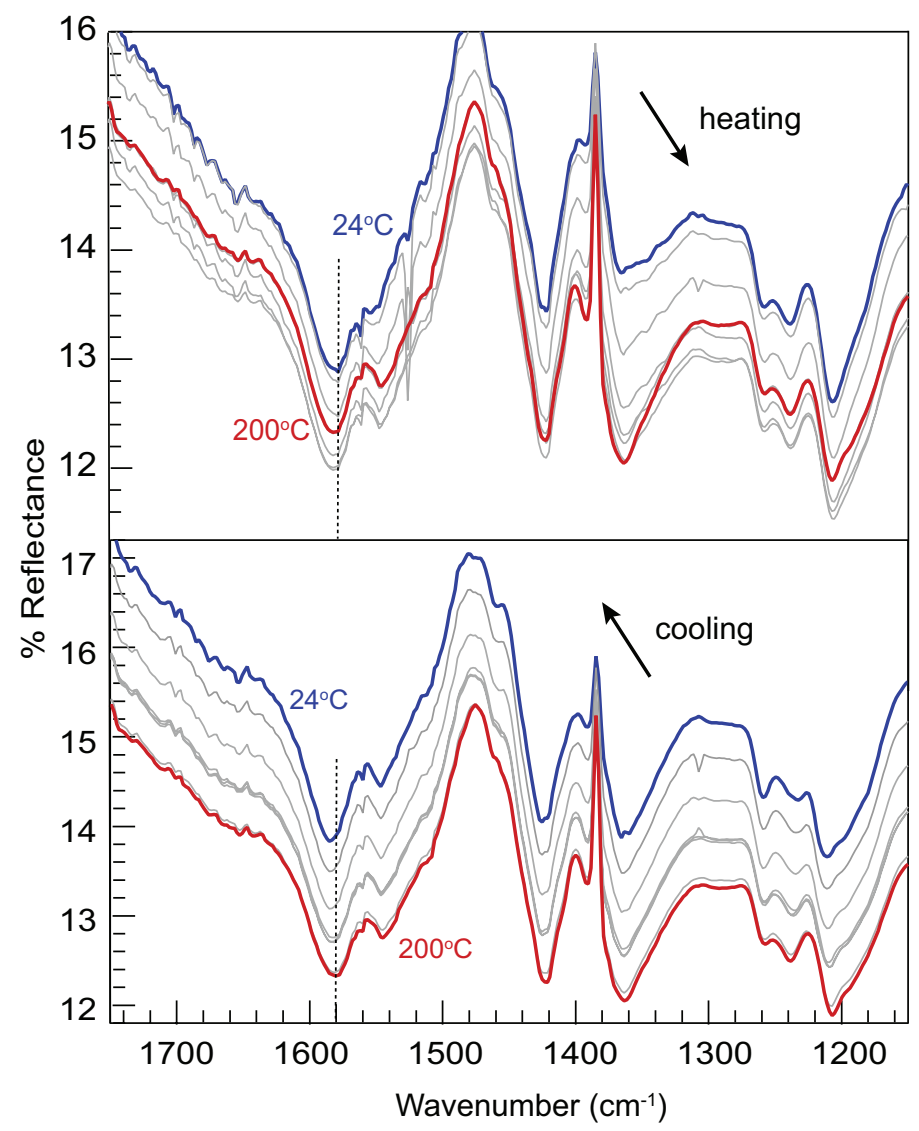

Figure S35: Unnormalized VT-DRIFTS of Cu-MOF-74. The symmetric stretch appears at 1360 $\mathrm{cm}^{-1}$ and asymmetric at $1580 \mathrm{~cm}^{-1}$. Interestingly, the asymmetric stretch continuously blueshifts throughout the measurement (initial minimum at room temperature $24^{\circ} \mathrm{C}-1577.5 \mathrm{~cm}^{-1}$; after heating to $200^{\circ} \mathrm{C}-1581.3 \mathrm{~cm}^{-1}$; after cooling to $24^{\circ} \mathrm{C}-1585.2 \mathrm{~cm}^{-1}$ ). 


\section{Computational Methods}

Density Functional Theory (DFT) and finite difference method (FDM) calculations were performed to identify the vibrational frequencies of different MOF systems.

Structural optimization for all structures were performed with DFT as implemented in the Vienna $\mathrm{ab}$ initio Simulation Package (VASP, version 5.4.4). ${ }^{10}$ All structures were equilibrated using the unrestricted GGA-PBEsol exchange-correlation functional ${ }^{11}$ except HKUST-1 (antiferromagnetic) where electron spins were set to pair up within each $\mathrm{Cu}$ dimer. Ionic relaxation was achieved when all forces were smaller than $0.005 \mathrm{eV} \AA^{-1}$. The plane-wave cut off was set at $500 \mathrm{eV}$, and the SCF convergence criterion was $10^{-6} \mathrm{eV}$, resulting in electronic convergence of $0.005 \mathrm{eV}$ per atom. An automatic k-grid was used during the optimization with $2 \times 2 \times 2$ sampling, except those for MOF-74 where the k-grid was set to be $1 \times 1 \times 4$. Symmetry was not enforced. Each optimized structure was then subjected to a single point calculation to obtain the predicted wavefunctions.

From the equilibrated structures, vibrational frequencies were obtained via finite differences method implemented in VASP where zone-center ( $\Gamma$-point) frequencies were calculated. Central difference was enforced to allow both negative and positive displacements of ions and the step size was set to be $0.015 \AA$ as default. The unrestricted GGA-PBEsol exchange-correlation functional were used with all convergence criteria similar to those of the optimization calculations. A scale factor of 0.0001 was enforced on the vector field for vibrational frequencies visualization. 
Sodium Benzoate


Frequency $\left(\mathrm{cm}^{-1}\right)$
a) 1603
Predicted mode
Frequency $\left(\mathrm{cm}^{-1}\right)$
Predicted mode
b) 1596
$\mathrm{C}-\mathrm{H}$ bend, $\mathrm{C}=\mathrm{C}$ stretch
e) 1427
$\mathrm{C}-\mathrm{H}$ bend, C-C zig-zag
f) 1384
OCO asym., $\mathrm{C}-\mathrm{H}$ bend
g) 1371
d) 1467
$\mathrm{C}-\mathrm{H}$ bend
h) 1272
$\mathrm{C}-\mathrm{H}$ bend
OCO sym., C-H bend
$\mathrm{C}=\mathrm{C}$ stretch, $\mathrm{C}-\mathrm{H}$ bend
$\mathrm{C}-\mathrm{H}$ bend

Predicted vibration frequencies with their vibrational modes for Sodium Benzoate:

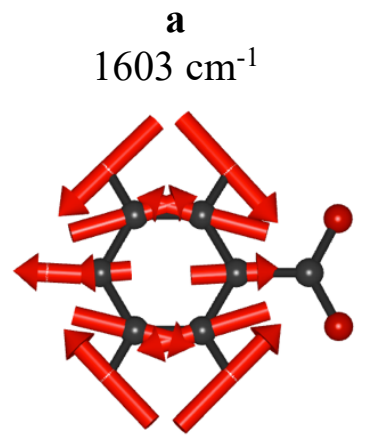

e

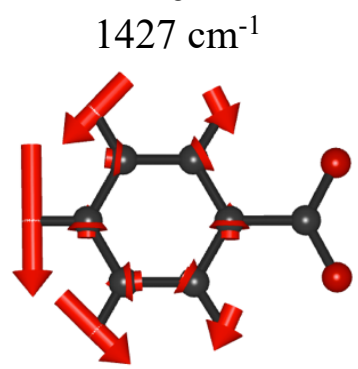

b

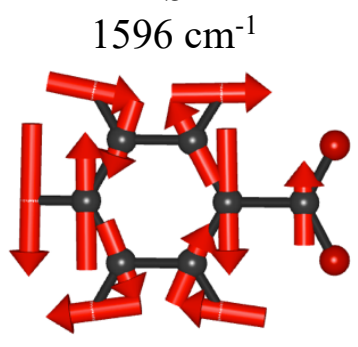

f

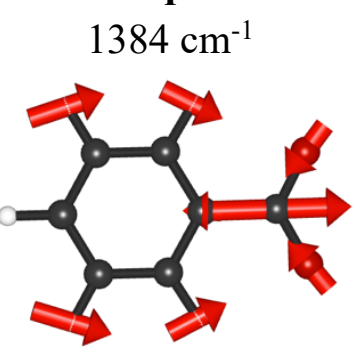

c

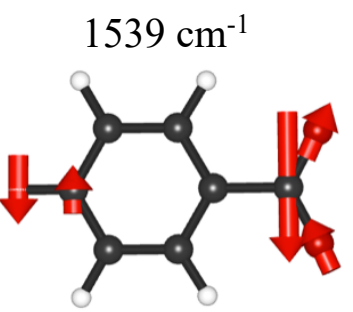

g

$1371 \mathrm{~cm}^{-1}$



d

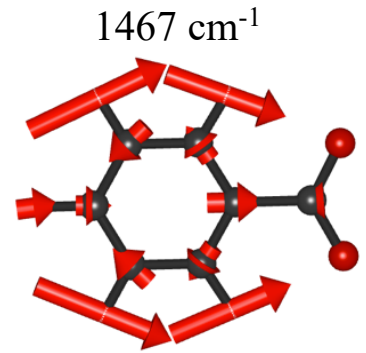

h $1272 \mathrm{~cm}^{-1}$

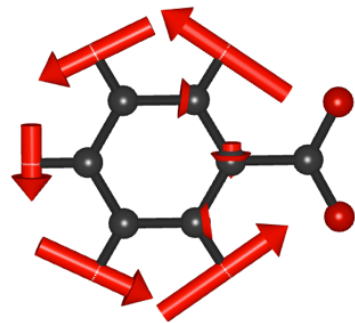

Figure S36. Summary of phonon mode calculations for sodium benzoate. 


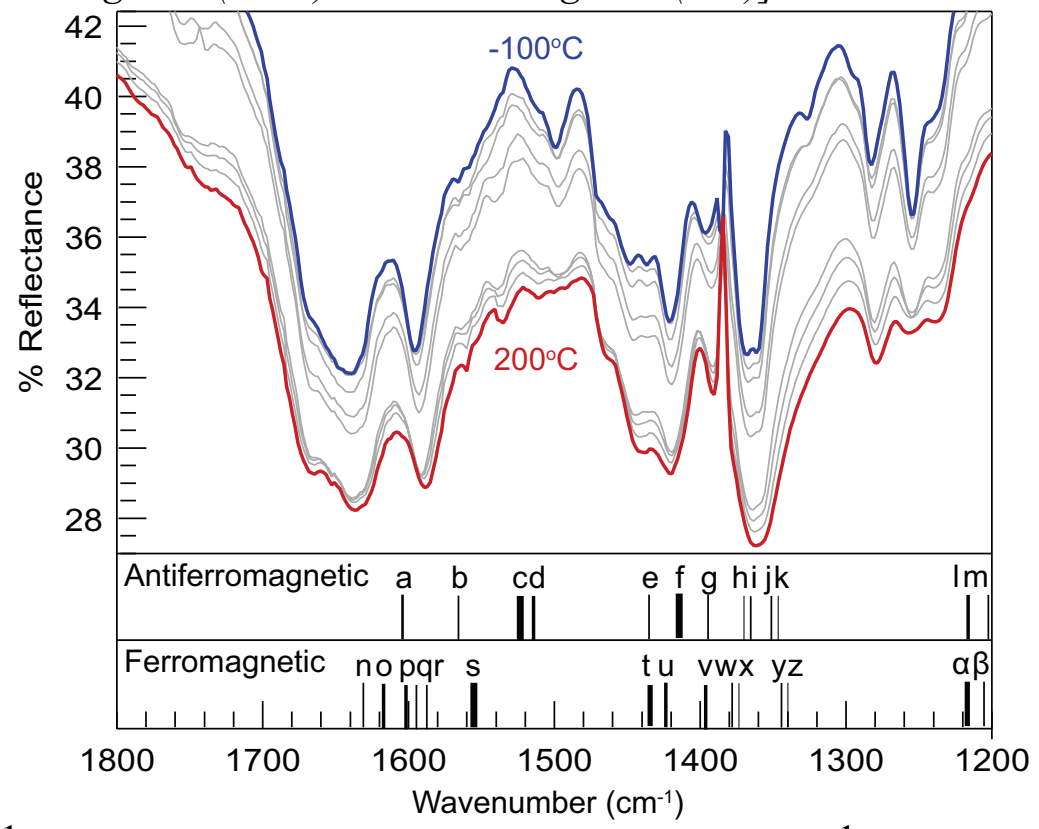

Frequency $\left(\mathrm{cm}^{-1}\right) \quad$ Predicted mode (AFM) Frequency $\left(\mathrm{cm}^{-1}\right)$ Predicted mode (AFM)

a) $1605-1600$

b) $1563-1560$

c) $1530-1523$

d) $1521-1518$

e) $1434-1431$

f) $1414,1412-1407$
$\mathrm{C}-\mathrm{H}$ bend, $\mathrm{C}=\mathrm{C}$ stretches

OCO asym.

OCO asym.

OCO asym.

$\mathrm{C}-\mathrm{H}$ bend

OCO sym. g) 1393-1392

h) 1365

i) $1359-1358$

j) $1347-1346$

k) 1336

l) $1215-1212$

m) $1203-1202$
$\mathrm{C}=\mathrm{C}$ stretching

$\mathrm{C}-\mathrm{H}$ bend, OCO sym.

$\mathrm{C}-\mathrm{H}$ bend, OCO sym.

C-H bend, OCO sym.

$\mathrm{C}-\mathrm{H}$ bend, OCO sym.

C-C stretch

$\mathrm{C}-\mathrm{H}$ bend 
Predicted vibration frequencies with their vibrational modes for HKUST-1 (AFM) below 348 K:

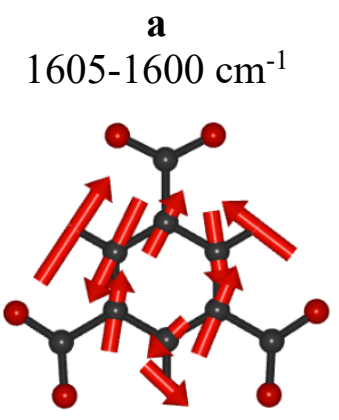

e

$1434-1431 \mathrm{~cm}^{-1}$



$1359-1358 \mathrm{~cm}^{-1}$

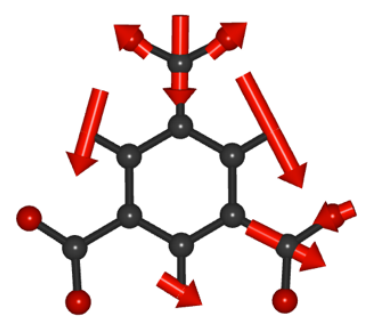

m

$1203-1202 \mathrm{~cm}^{-1}$

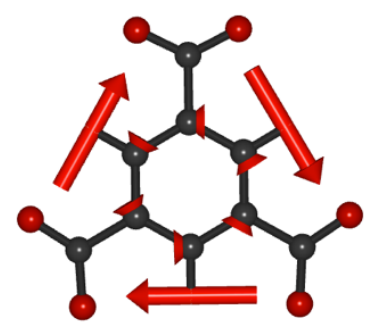

b

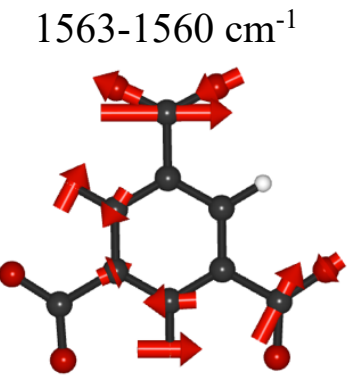

f

1414-1407 $\mathrm{cm}^{-1}$

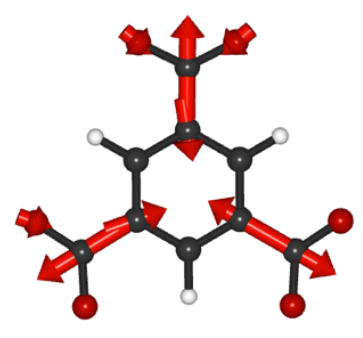

j

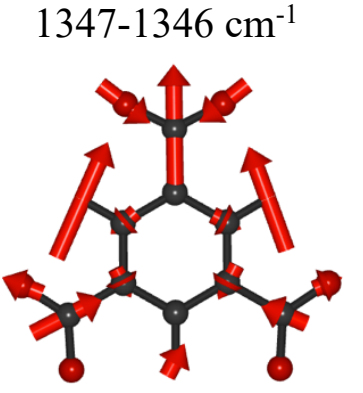

c

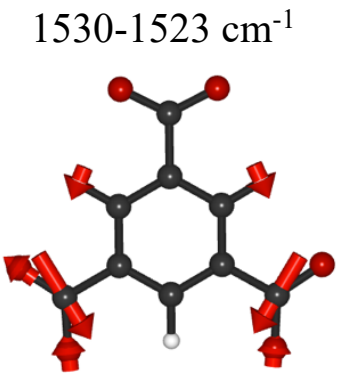

g

$1393-1392 \mathrm{~cm}^{-1}$

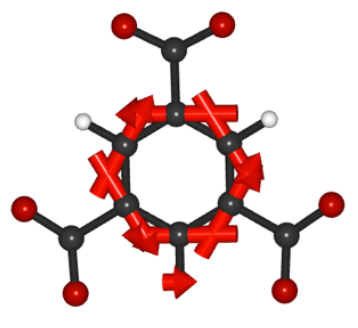

k

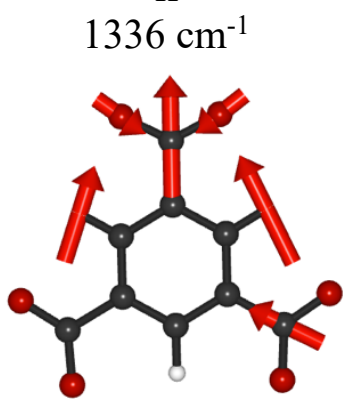

d

$1521-1518 \mathrm{~cm}^{-1}$

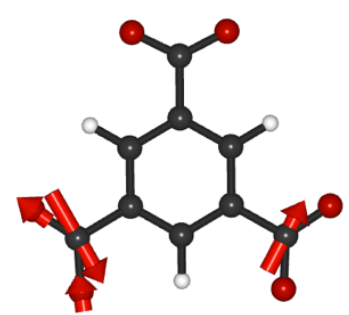

h

$1365 \mathrm{~cm}^{-1}$

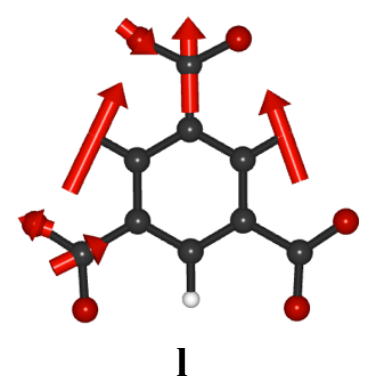

$1215-1212 \mathrm{~cm}^{-1}$

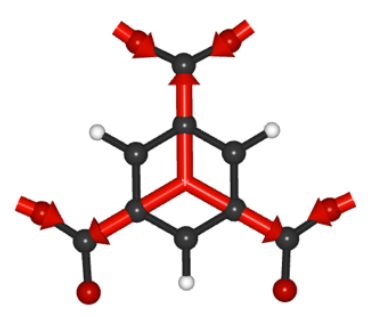




\begin{tabular}{|c|c|c|c|}
\hline $\begin{array}{l}\text { Frequency } \\
\left(\mathrm{cm}^{-1}\right)\end{array}$ & $\begin{array}{l}\text { Predicted mode } \\
\text { (FM) }\end{array}$ & $\begin{array}{l}\text { Frequency } \\
\left(\mathrm{cm}^{-1}\right)\end{array}$ & $\begin{array}{l}\text { Predicted mode } \\
\text { (FM) }\end{array}$ \\
\hline n) 1634 & OCO asym., $\mathrm{C}=\mathrm{C}$ stretch & v) $1395-1394$ & $\mathrm{C}=\mathrm{C}$ stretches \\
\hline o) 1621-1620 & OCO asym., C-H bend & w) 1375 & C-H bend, OCO sym. \\
\hline p) $1602-1601$ & $\mathrm{C}-\mathrm{H}$ bend, $\mathrm{C}=\mathrm{C}$ stretches & x) 1367 & $\mathrm{C}-\mathrm{H}$ bend, OCO sym. \\
\hline q) 1594 & C-H bend, C-C zig-zag & y) $1348-1347$ & $\mathrm{C}-\mathrm{H}$ bend, OCO sym. \\
\hline r) 1587 & C-H bend, C-C zig-zag & z) 1339 & $\mathrm{C}-\mathrm{H}$ bend, OCO sym. \\
\hline s) $1523-1515$ & OCO asym. & a) $1217-1213$ & C-C stretch \\
\hline t) $1437-1430$ & $\mathrm{C}-\mathrm{H}$ bend, $\mathrm{C}=\mathrm{C}, \mathrm{C}-\mathrm{C}$ stretches & ק) $1204-1203$ & $\mathrm{C}-\mathrm{H}$ bend \\
\hline u) $1423-1421$ & OCO sym., C-C stretch & & \\
\hline
\end{tabular}


Simulated phonon modes for HKUST-1 (FM) above $280 \mathrm{~K}$ :

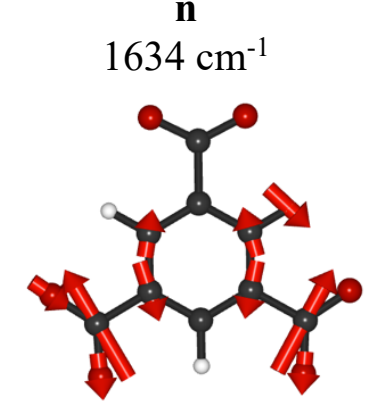

r

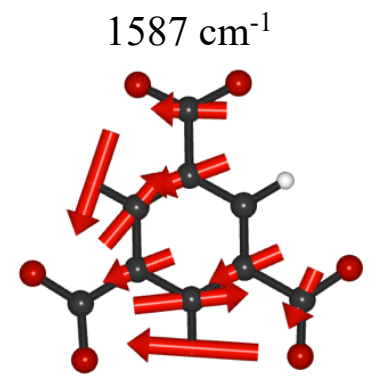

v

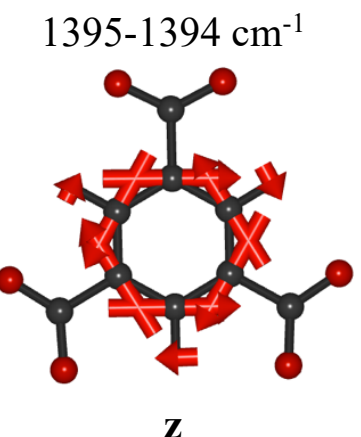

$1339 \mathrm{~cm}^{-1}$

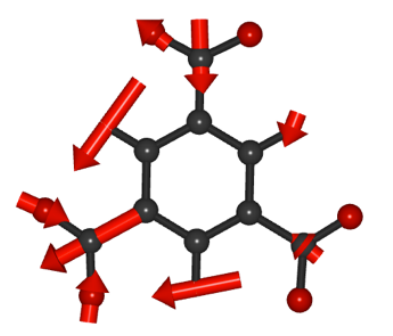

o

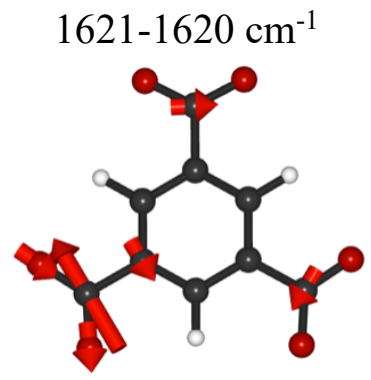

S

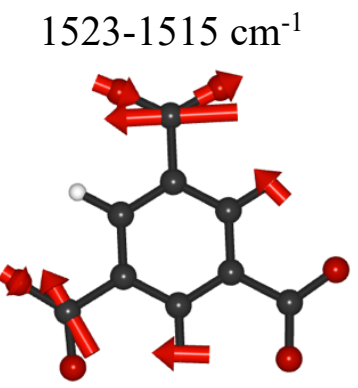

$\mathbf{w}$

$1375 \mathrm{~cm}^{-1}$

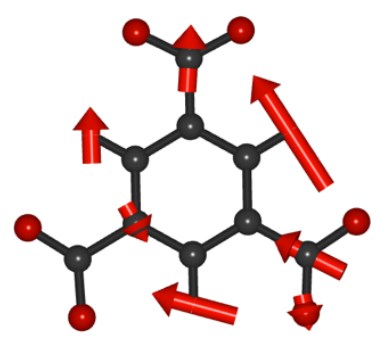

$\alpha$

$1217-1213 \mathrm{~cm}^{-1}$

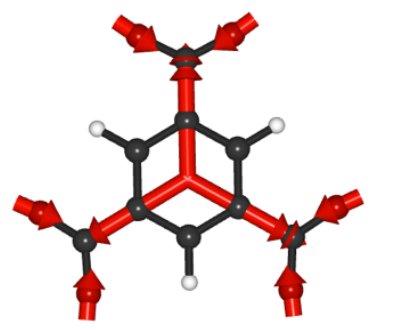

p



t

$1437-1430 \mathrm{~cm}^{-1}$

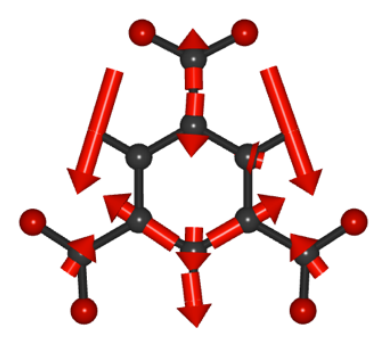

$\mathbf{x}$

$1367 \mathrm{~cm}^{-1}$

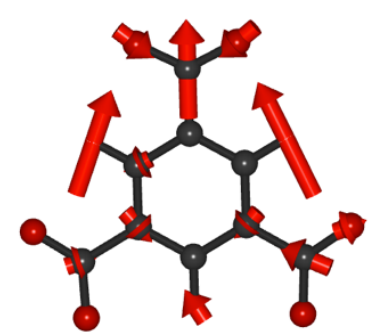

$\beta$

$1204-1203 \mathrm{~cm}^{-1}$

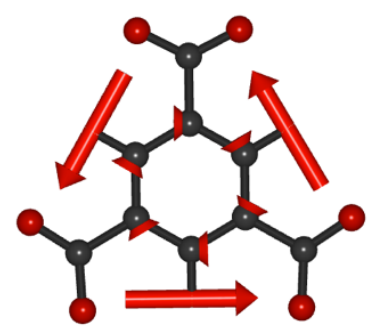

Figure S37. Summary of phonon mode calculations for HKUST-1 in AFM and FM regimes. 
A


Frequency $\left(\mathrm{cm}^{-1}\right)$ Predicted mode
a) 1671
1658
1649
1643
b) $1616-1614$
1610
c) 1602
d) $1593-1592$
1587,1585
1583
1579
e) 1517
f) 1505
g) 1493-1492
C-O stretch
C-O stretch
C-O stretch
C-O stretch
$\mathrm{C}-\mathrm{H}$ bend, $\mathrm{C}-\mathrm{O}$ stretch
$\mathrm{C}-\mathrm{H}$ bend, $\mathrm{C}-\mathrm{O}$ stretch
C-O stretch
$\mathrm{C}-\mathrm{H}$ bend
$\mathrm{C}-\mathrm{H}$ bend, $\mathrm{C}-\mathrm{O}$ stretch
$\mathrm{C}-\mathrm{H}$ bend, $\mathrm{C}-\mathrm{O}$ stretch
$\mathrm{C}-\mathrm{H}$ bend, $\mathrm{C}-\mathrm{O}$ stretch
$\mathrm{HOH}$ bend sym.
$\mathrm{HOH}$ bend sym.
$\mathrm{HOH}$ bend sym.

Frequency $\left(\mathrm{cm}^{-1}\right)$ Predicted mode

g) 1488-1487 HOH bend sym.

h) 1433-1429 C-H bend, C-C stretch

i) $1425-1423 \quad \mathrm{C}-\mathrm{H}$ bend

j) 1409

k) $1395-1394$

1) $1389-1388$

O-C-C stretch

$\mathrm{O}-\mathrm{C}-\mathrm{C}$ stretch

$\mathrm{C}=\mathrm{C}$ stretch

m) 1370

n) 1346

o) 1313

1306

1303

p) 1221

1216

1212-1211
$\mathrm{C}-\mathrm{H}$ bend, C-O stretch

$\mathrm{C}-\mathrm{H}$ bend, $\mathrm{C}-\mathrm{O}$ stretch

$\mathrm{C}-\mathrm{H}$ bend, C-O stretch

$\mathrm{C}-\mathrm{H}$ bend, C-O stretch

$\mathrm{C}-\mathrm{H}$ bend, C-O stretch

C-C stretch

C-C stretch, OCO sym., C-H bend C-C stretch, OCO sym., C-H bend 
Predicted vibration frequencies with their vibrational modes for MUV-10:

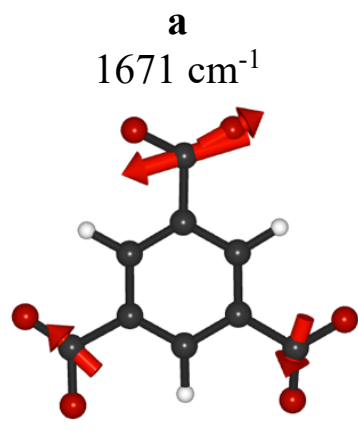

b

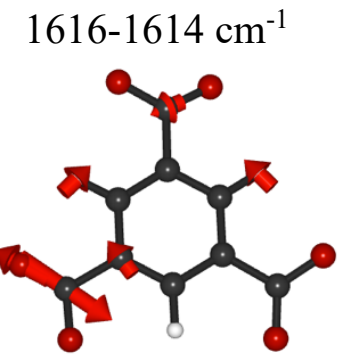

d

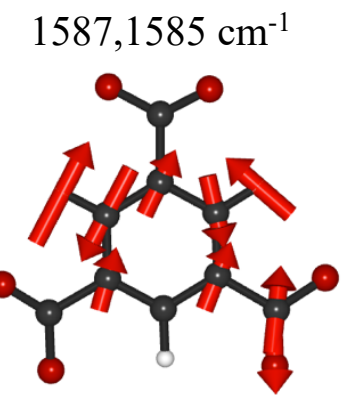

f

$1505 \mathrm{~cm}^{-1}$

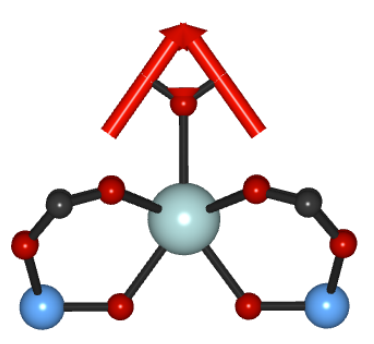

i

$1425-1423 \mathrm{~cm}^{-1}$

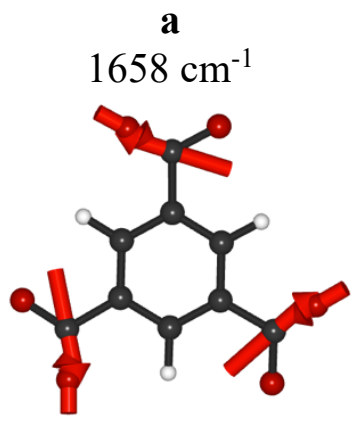

b

$1610 \mathrm{~cm}^{-1}$

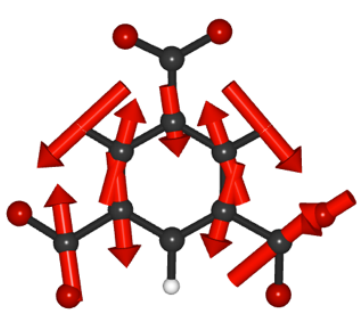

d

$1583 \mathrm{~cm}^{-1}$

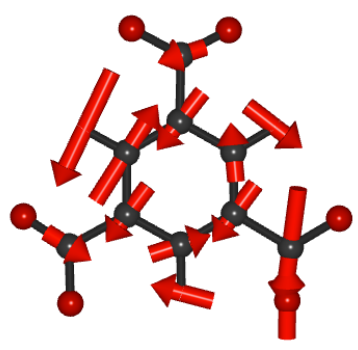

g

$1493-1492 \mathrm{~cm}^{-1}$



$\underset{1409 \mathrm{~cm}^{-1}}{\mathbf{j}}$



c

$1602 \mathrm{~cm}^{-1}$

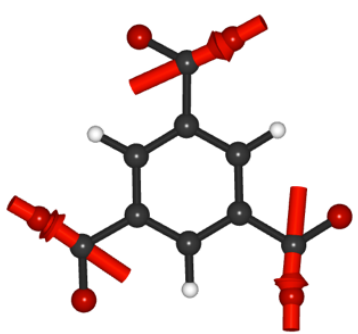

d

$1579 \mathrm{~cm}^{-1}$

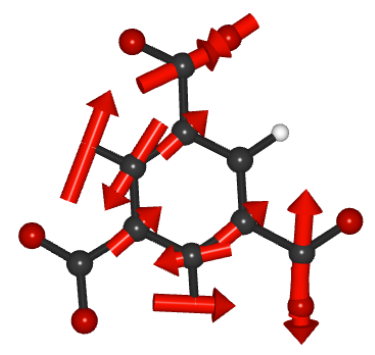

g

$1488-1487 \mathrm{~cm}^{-1}$

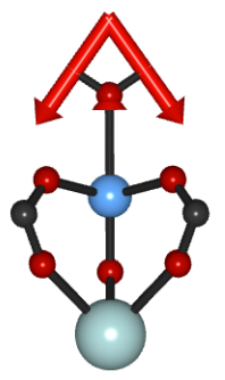

k

$1395-1394 \mathrm{~cm}^{-1}$

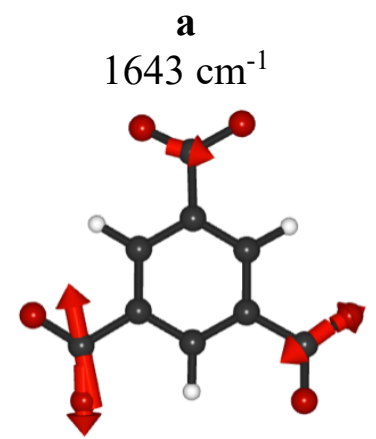

d $1593-1592 \mathrm{~cm}^{-1}$

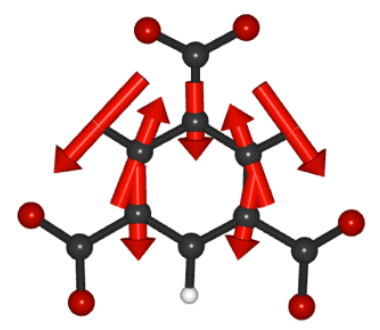

e

$1517 \mathrm{~cm}^{-1}$

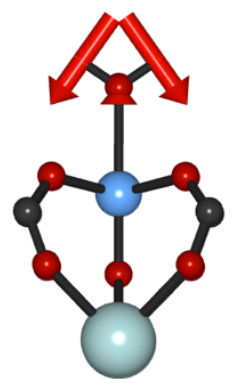

h

$1433-1429 \mathrm{~cm}^{-1}$

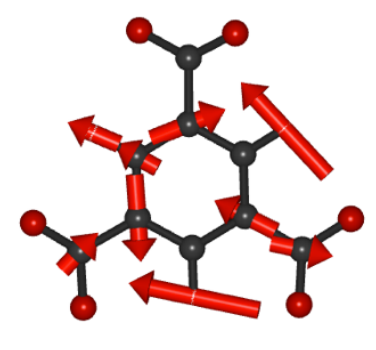

I

$1389-1388 \mathrm{~cm}^{-1}$ 




m

$1370 \mathrm{~cm}^{-1}$

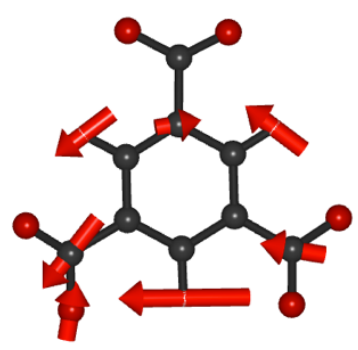

o

$1303 \mathrm{~cm}^{-1}$

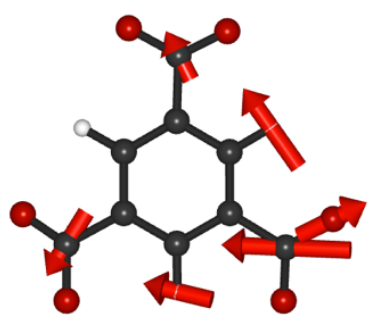



n

$1346 \mathrm{~cm}^{-1}$

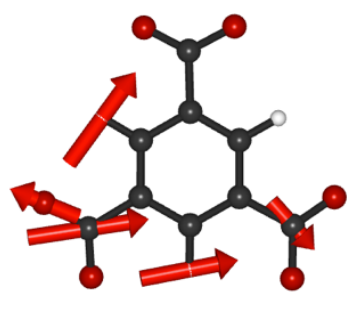

p

$1221 \mathrm{~cm}^{-1}$

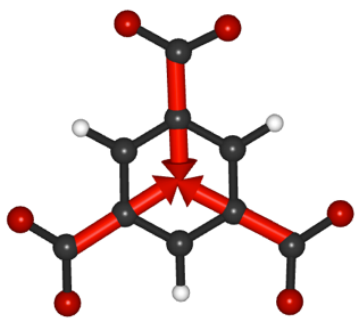



0

$1313 \mathrm{~cm}^{-1}$

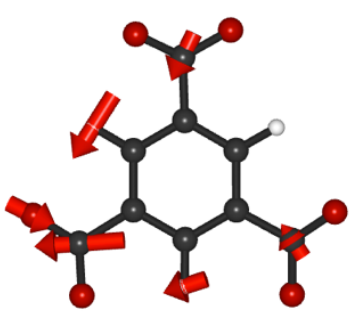

p

$1216 \mathrm{~cm}^{-1}$

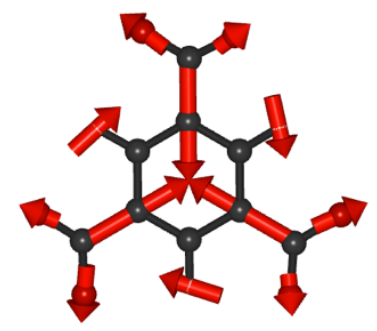

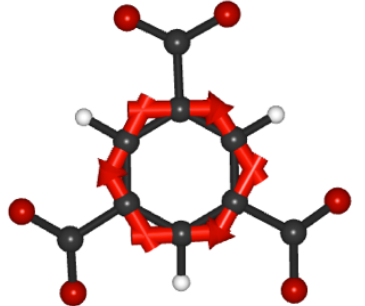

0

$1306 \mathrm{~cm}^{-1}$

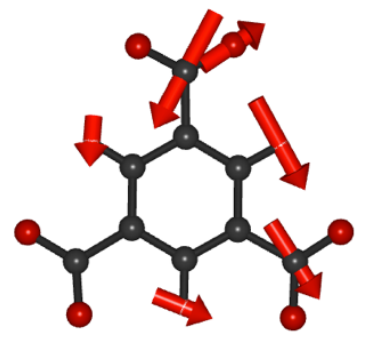

p

$1212-1211 \mathrm{~cm}^{-1}$

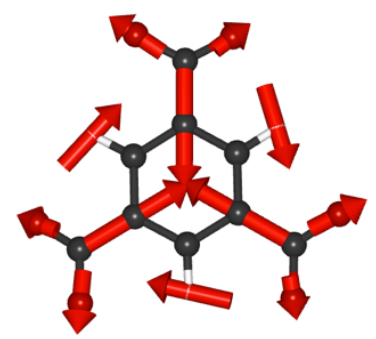

Figure S38. Summary of phonon mode calculations for (A) MUV-10 (Ca) and (B) MUV-10 (Mn). 

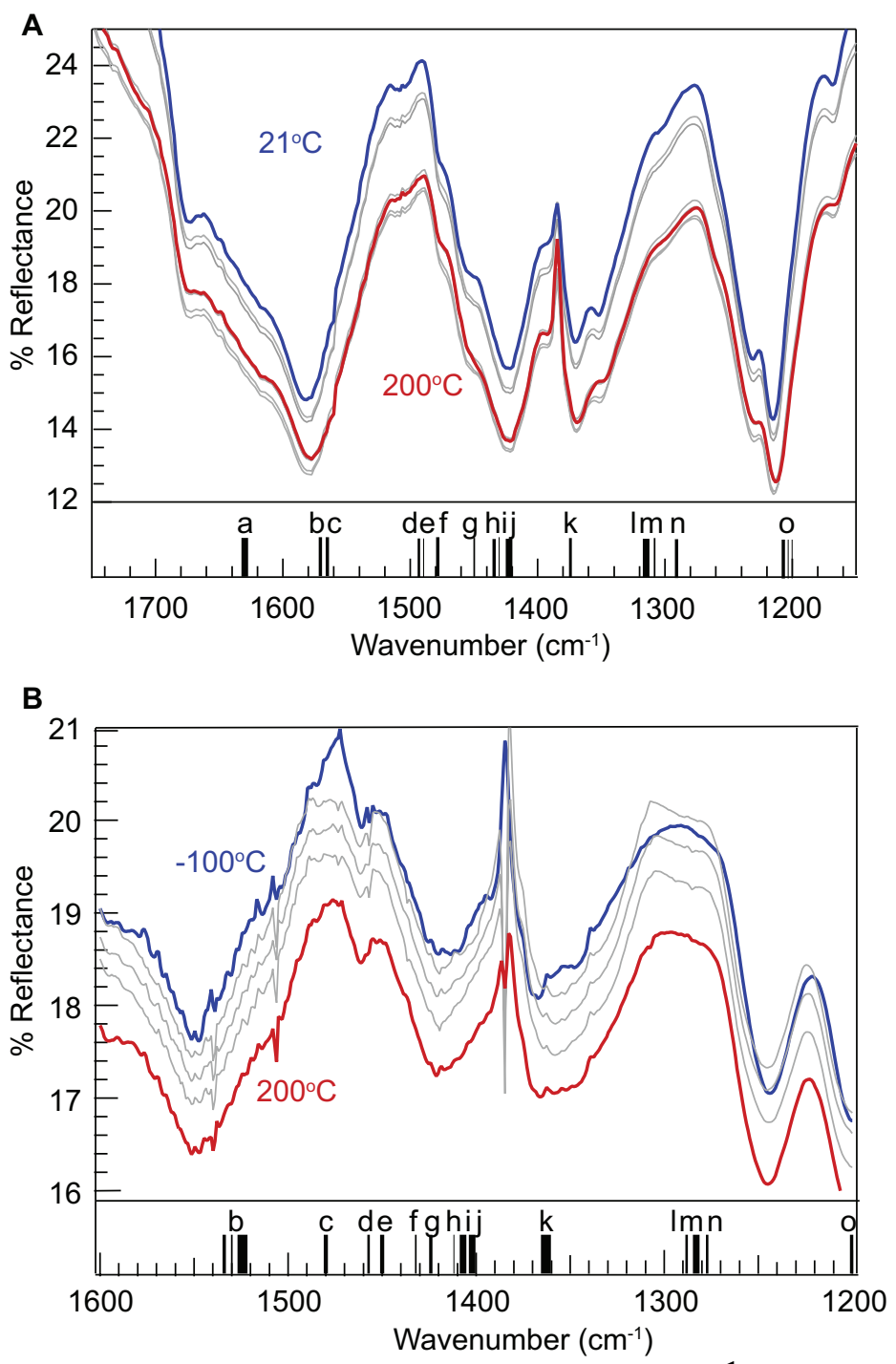

Frequency $\left(\mathrm{cm}^{-1}\right)$ Predicted mode
a) $1630-1626$
b) $1573-1571$
c) $1568-1566$
d) $1493-1491$
e) 1487
f) $1479-1477$
g) $1451-1450$
h) $1439-1437$

$\mathrm{C}-\mathrm{H}$ bend

OCO asym.

OCO asym.

$\mathrm{C}=\mathrm{C}$ zig-zag, $\mathrm{C}-\mathrm{H}$ stretch

OCO sym.

$\mathrm{C}-\mathrm{H}$ bend, OCO sym.

$\mathrm{C}-\mathrm{H}$ bend

$\mathrm{C}-\mathrm{H}$ bend

\section{Frequency $\left(\mathrm{cm}^{-1}\right)$}

i) 1434

j) $1428-1422$

k) $1378-1376$

1,m)1318-1313, 1309

n) $1293-1290$

o) 1208-1206

1203

1200

\section{Predicted mode}

OCO sym.

OCO sym.

OCO sym., $\mathrm{C}=\mathrm{C}$ stretches 
Predicted vibration frequencies with their vibrational modes for Mg-MOF-74:

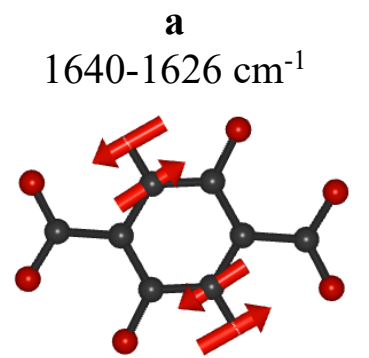

e

$1487 \mathrm{~cm}^{-1}$ b

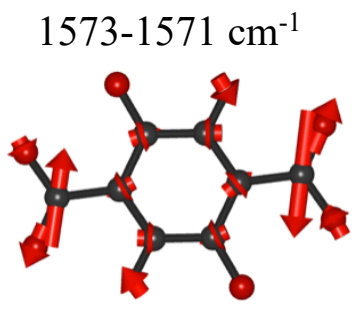

f

$1479-1477 \mathrm{~cm}^{-1}$ c

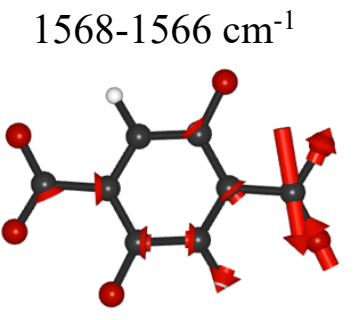

g

$1451-1450 \mathrm{~cm}^{-1}$ d $1493-1491 \mathrm{~cm}^{-1}$



h $1439-1437 \mathrm{~cm}^{-1}$
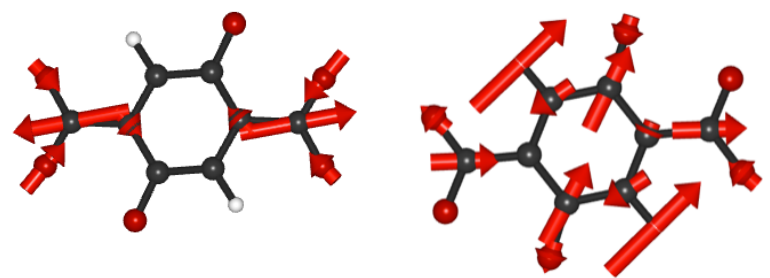

i

$1434 \mathrm{~cm}^{-1}$

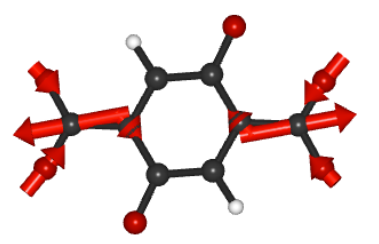

$1428-1422 \mathrm{~cm}^{-1}$



n

o

$1208-1206 \mathrm{~cm}^{-1}$
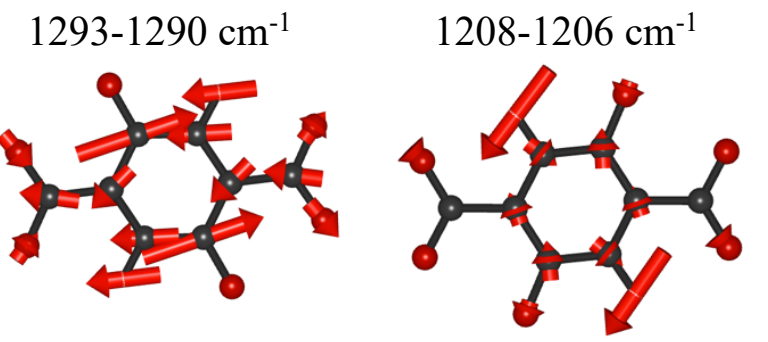

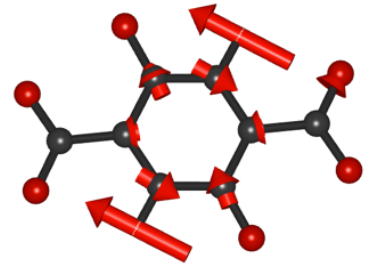

k

$1378-1376 \mathrm{~cm}^{-1}$

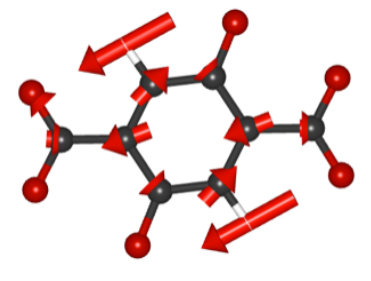

$\mathbf{l , m}$

$1318-1309 \mathrm{~cm}^{-1}$

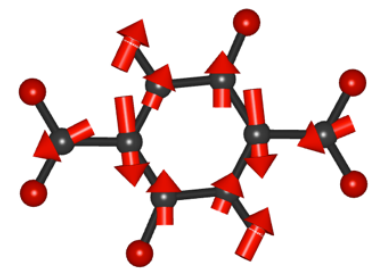

o

$1203 \mathrm{~cm}^{-1}$

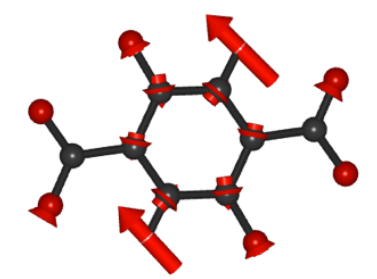

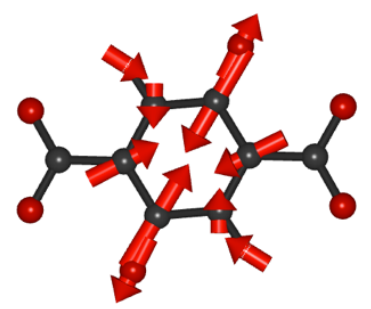

o

$1200 \mathrm{~cm}^{-1}$

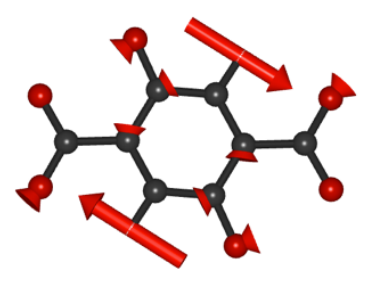


Predicted vibration frequencies with their vibrational modes for $\mathrm{Zn-MOF-74:}$

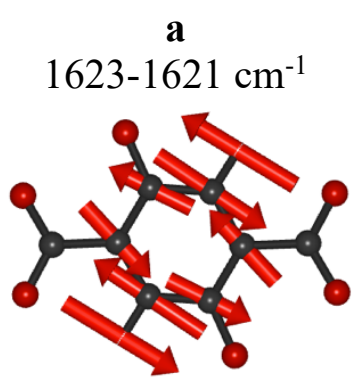

c

$1493-1491 \mathrm{~cm}^{-1}$

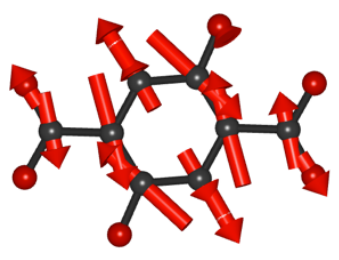

g

$1435-1434 \mathrm{~cm}^{-1}$

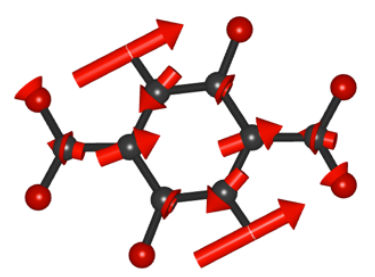

$\mathbf{k}$

$1376-1371 \mathrm{~cm}^{-1}$

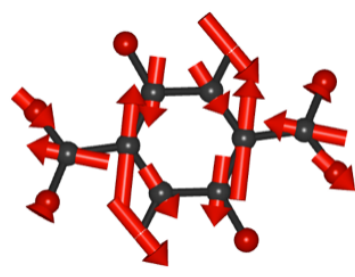

o

$1202-1201 \mathrm{~cm}^{-1}$



b

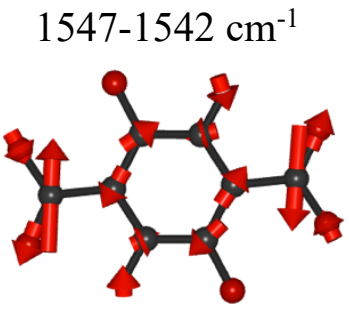

d

$1469 \mathrm{~cm}^{-1}$



h

$1422 \mathrm{~cm}^{-1}$

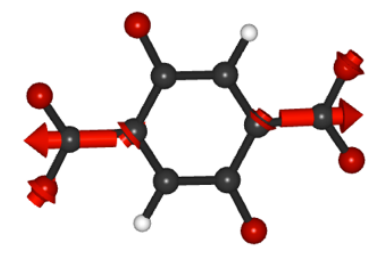

I

$1298-1297 \mathrm{~cm}^{-1}$

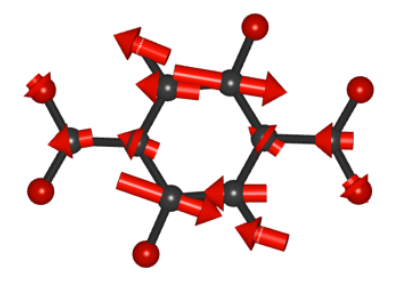

$1540-1538 \mathrm{~cm}^{-1}$

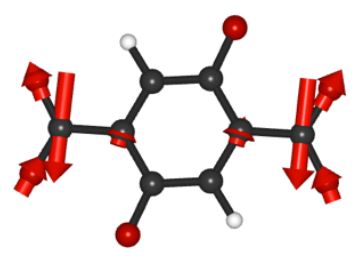

e

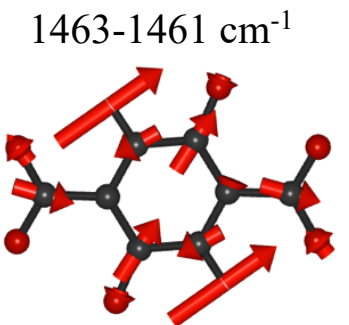

i

$1418-1416 \mathrm{~cm}^{-1}$

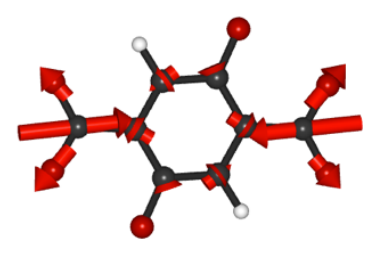

m

$1294-1291 \mathrm{~cm}^{-1}$

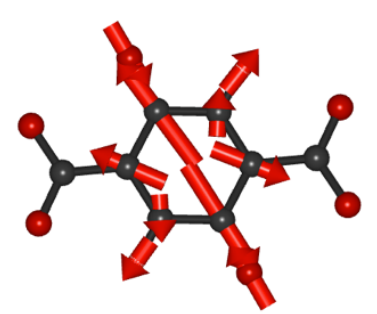

b

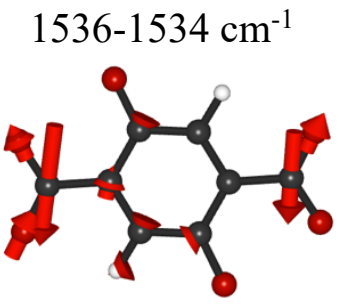

f $1442 \mathrm{~cm}^{-1}$

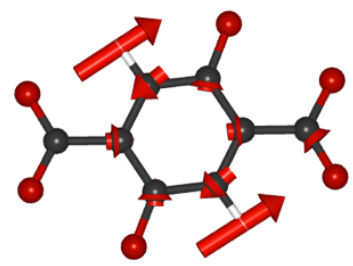

j

$1414-1412 \mathrm{~cm}^{-1}$



n

1287-1286 cm-1

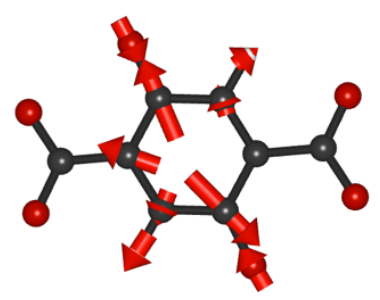

Figure S39. Summary of phonon mode calculations for (A) Mg-MOF-74 and (B) Zn-MOF-74. 
$M O F-5$

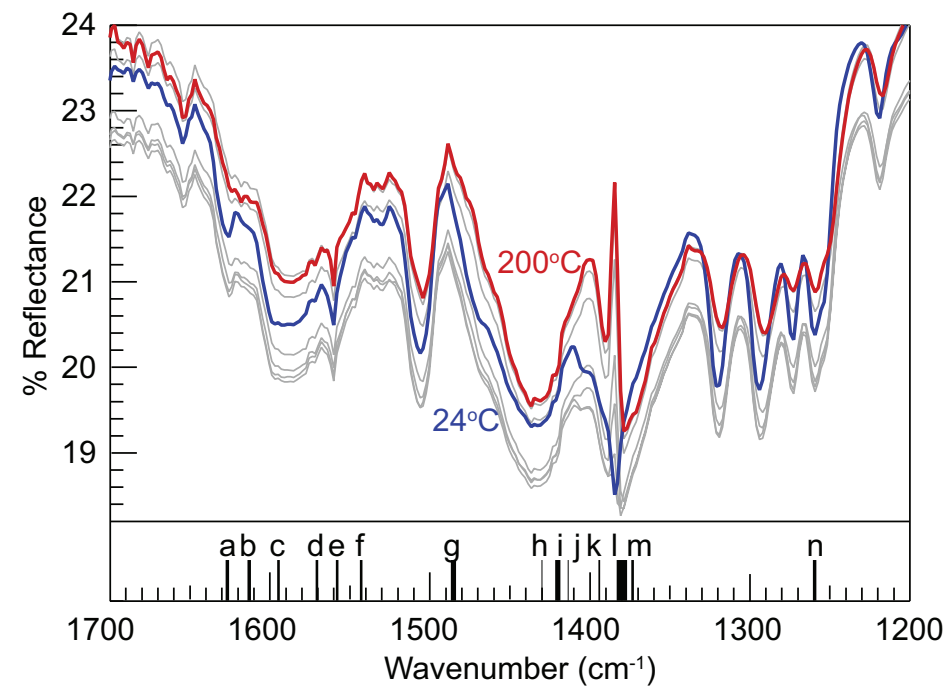

Frequency $\left(\mathrm{cm}^{-1}\right)$ Predicted mode

a) 1629-1628 OCO asym.

b) $1616-1615$

c) $1597-1596$

d) $1572-1571$

e) $1560-1559$

f) $1545-1544$

g) $1488-1585$
$\mathrm{C}-\mathrm{H}$ bend, $\mathrm{C}=\mathrm{C}$ stretch, $\mathrm{OCO}$ asym.

$\mathrm{C}-\mathrm{H}$ bend, C-C zig-zag

$\mathrm{C}-\mathrm{H}$ bend, OCO asym.

OCO asym.

OCO asym.

C-H bend, C-C stretch
Frequency $\left(\mathrm{cm}^{-1}\right)$ Predicted mode

h) 1430

i) $1422-1420$

j) 1413

k) 1396

l) $1384-1380$

m) $1375-1376$

n) $1260-1259$
OCO sym.

OCO sym., C-C stretch $\mathrm{C}-\mathrm{H}$ bend, OCO sym.

$\mathrm{C}-\mathrm{H}$ bend, $\mathrm{C}=\mathrm{C}$ stretch

$\mathrm{C}-\mathrm{H}$ bend

$\mathrm{C}-\mathrm{H}$ bend, OCO sym.

$\mathrm{C}-\mathrm{H}$ bend 
Predicted vibration frequencies with their vibrational modes for MOF-5:
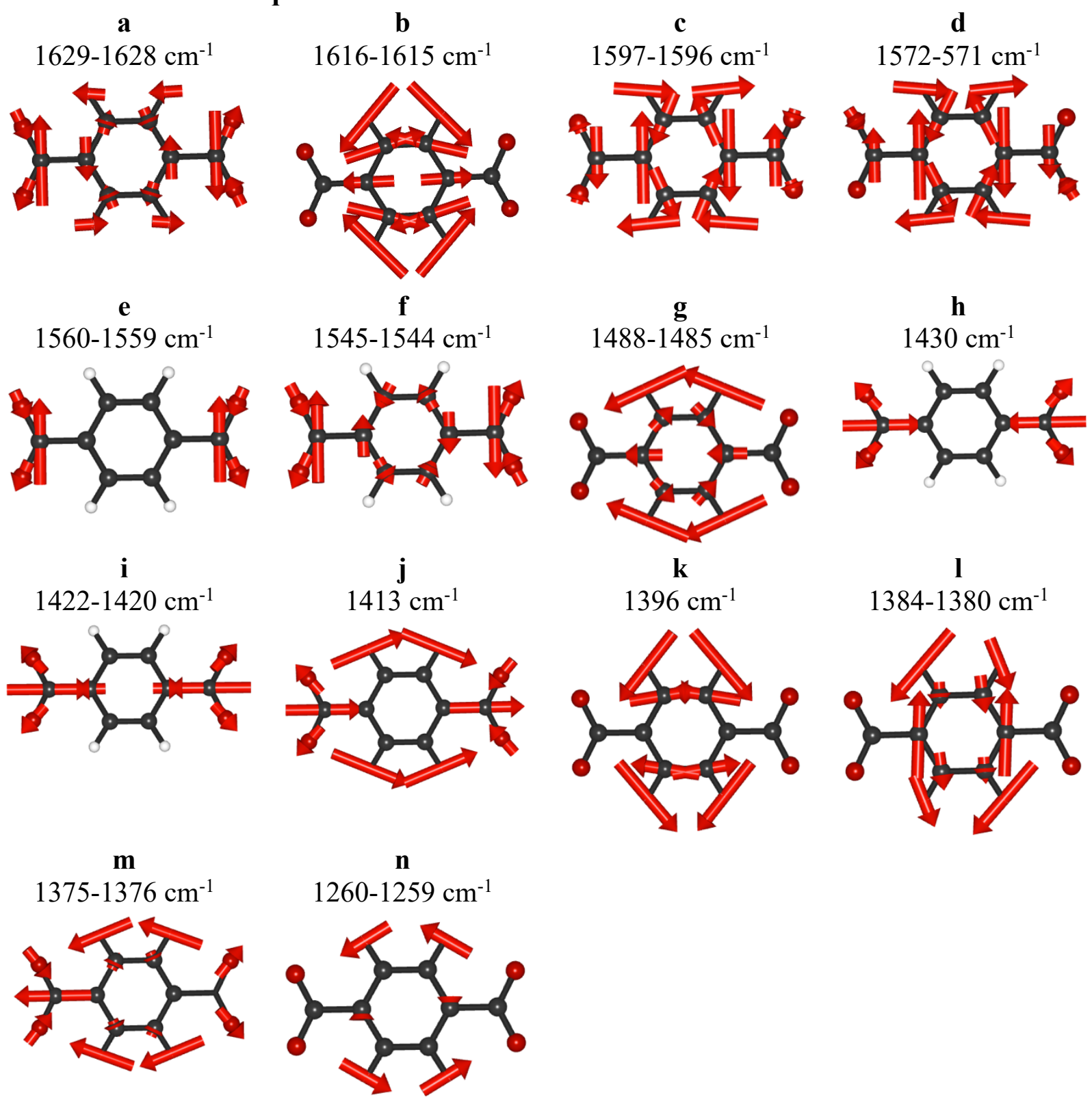

Figure S40. Summary of phonon mode calculations for MOF-5. 
MIL-125

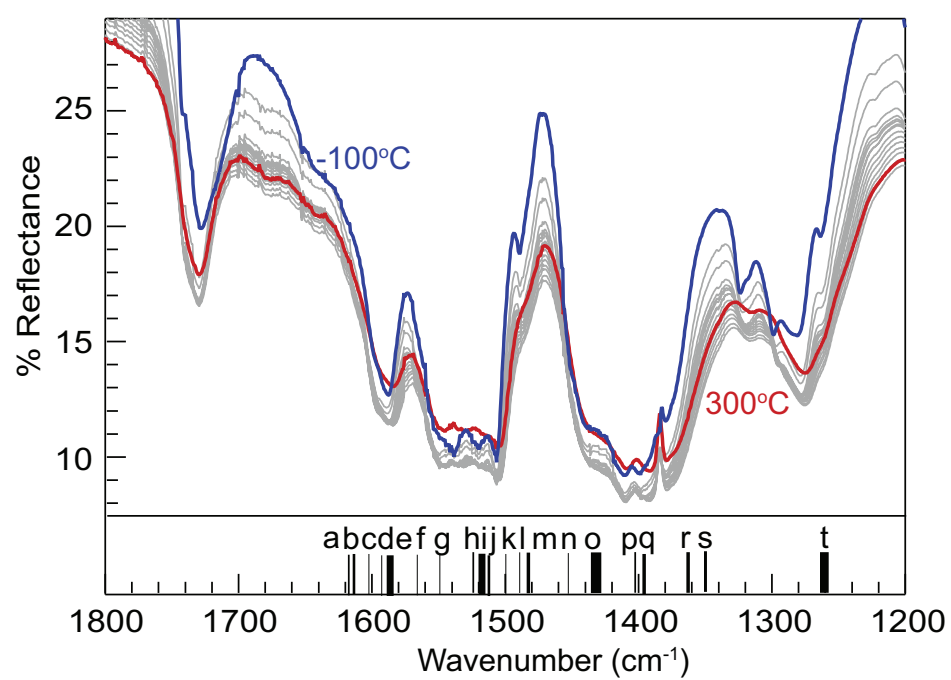

Frequency Predicted mode $\left(\mathrm{cm}^{-1}\right)$

a) 1618

b) $1615-1614$

c) 1602

d) $1591-1590$

e) $1589-1586$

f) 1564

g) 1552

h) 1528

i) $1524-1520$

j) $1511-1510$
OCO asym., C-H bend

$\mathrm{C}-\mathrm{H}$ bend, $\mathrm{C}=\mathrm{C}$ stretch

$\mathrm{OCO}$ asym., $\mathrm{C}-\mathrm{H}$ bend, $\mathrm{C}=\mathrm{C}$ stretch

OCO asym.

$\mathrm{C}-\mathrm{H}$ bend, C-C zig-zag

$\mathrm{C}-\mathrm{H}$ bend, OCO asym.

$\mathrm{C}-\mathrm{H}$ bend, OCO asym.

OCO asym.

OCO asym.

OCO asym.
Frequency $\left(\mathrm{cm}^{-1}\right)$

k) 1498

1) 1487

m) $1484-1481$

n) $1452 \quad$ OCO sym.

o) 1438-1428 OCO sym.

p) 1406-1405 OCO sym., C-H bend

q) $1398-1396$

r) $1383-1380$

s) $1376-1374$

t) $1263-1259$
Predicted mode

OCO asym.

$\mathrm{C}-\mathrm{H}$ bend

$\mathrm{C}-\mathrm{H}$ bend

$\mathrm{C}-\mathrm{H}$ bend, $\mathrm{C}=\mathrm{C}$ stretch $\mathrm{C}-\mathrm{H}$ bend, $\mathrm{C}=\mathrm{C}$ stretch OCO sym., C-H bend $\mathrm{C}-\mathrm{H}$ bend 
Predicted vibration frequencies with their vibrational modes for MIL-125:
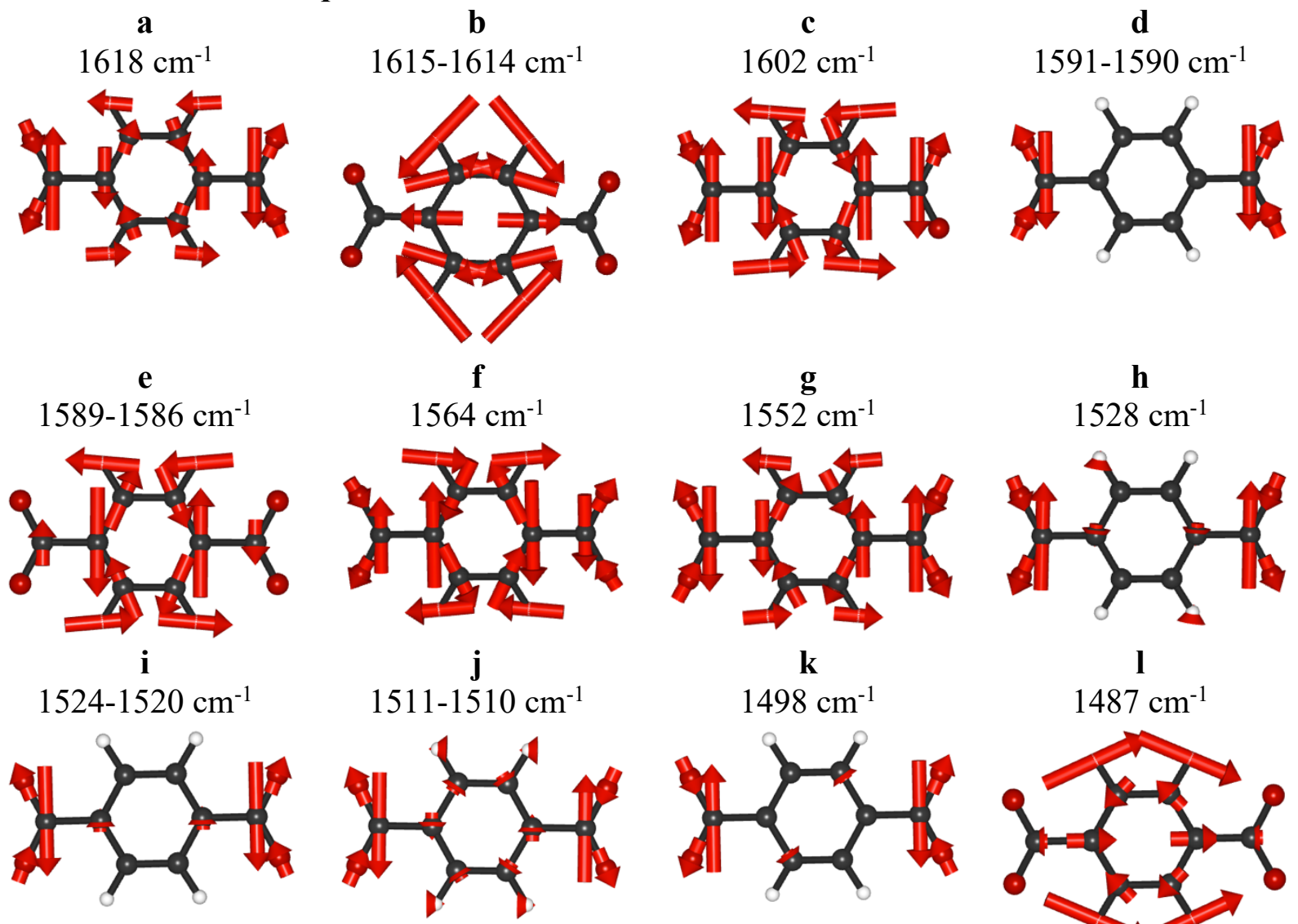

k

$1498 \mathrm{~cm}^{-1}$
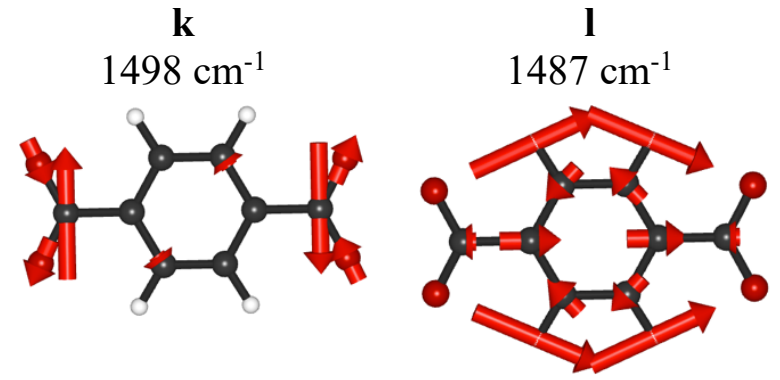

m

n

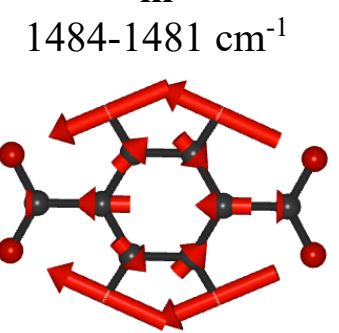

q

$1452 \mathrm{~cm}^{-1}$

о

$1438-1428 \mathrm{~cm}^{-1}$

p $1406-1405 \mathrm{~cm}^{-1}$


$\mathbf{r}$

S

$1398-1396 \mathrm{~cm}^{-1}$

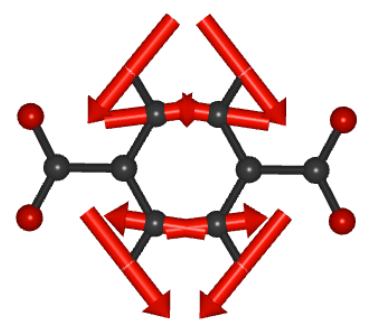

$1383-1380 \mathrm{~cm}^{-1}$

$1376-1374 \mathrm{~cm}^{-1}$
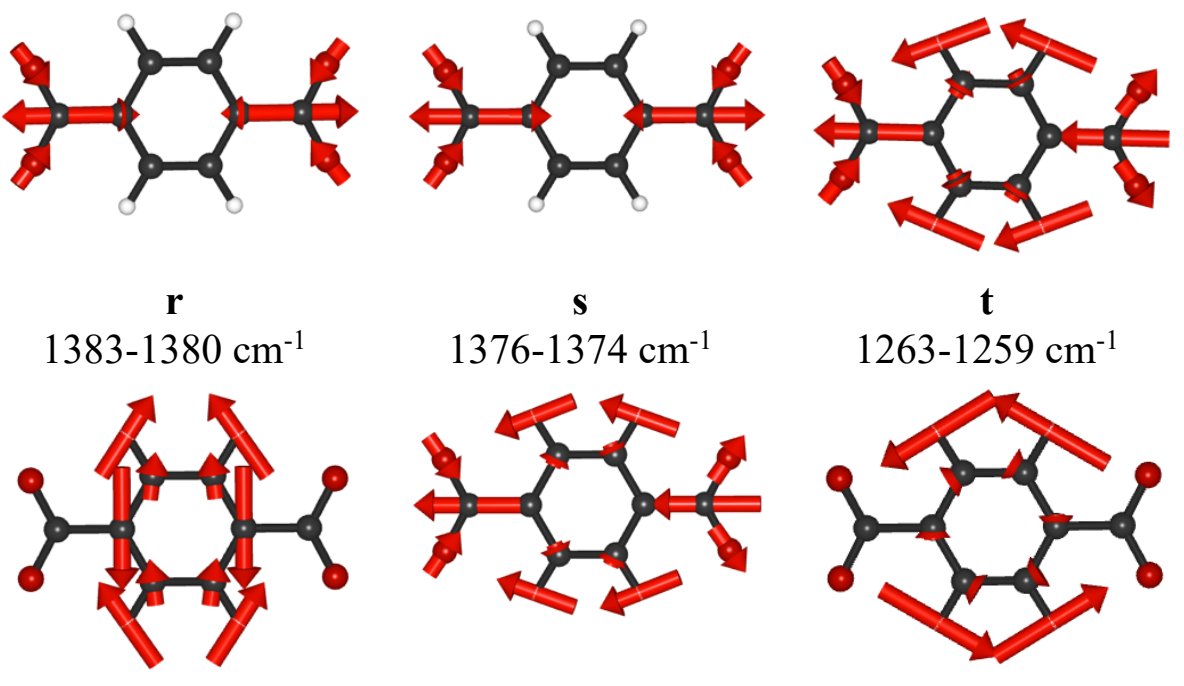

Figure S41. Summary of phonon mode calculations for MIL-125. 


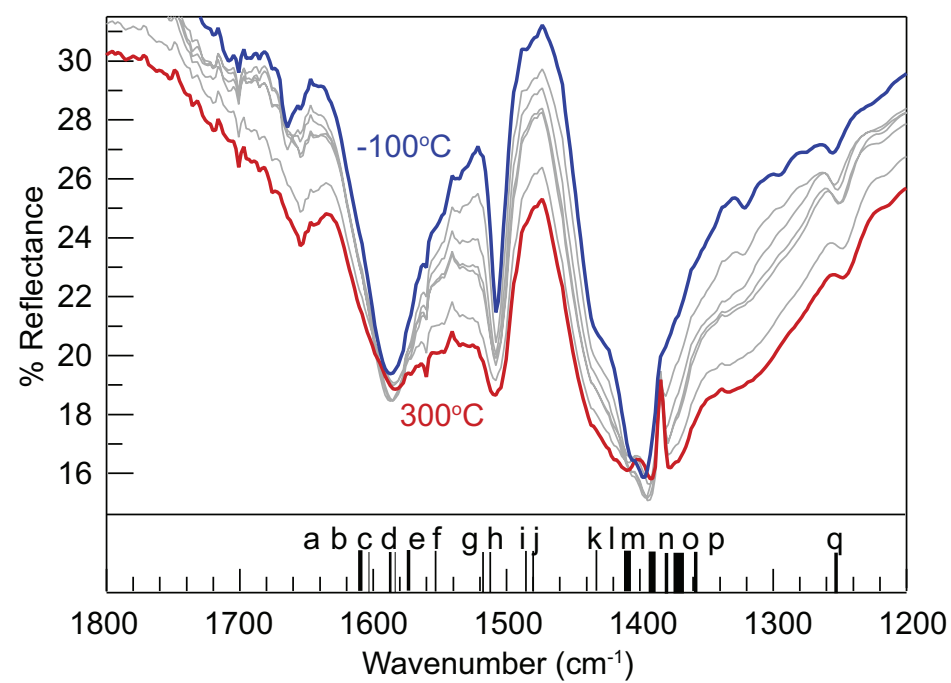

Frequency $\left(\mathrm{cm}^{-1}\right)$ Predicted mode
a) $1615-1613$
b) 1607
c) $1592-1591$
d) 1588
e) $1577-1576$
f) 1556
g) 1521
h) 1516
i) 1488

$\mathrm{C}-\mathrm{H}$ bend, $\mathrm{C}=\mathrm{C}$ stretch

OCO asym., C-H bend, $\mathrm{C}=\mathrm{C}$ stretch

$\mathrm{C}-\mathrm{H}$ bend, $\mathrm{C}-\mathrm{C}$ zig-zag

$\mathrm{C}-\mathrm{H}$ bend, $\mathrm{C}=\mathrm{C}$ stretch

OCO asym.

OCO asym., $\mathrm{C}-\mathrm{H}$ bend

OCO asym.

OCO asym.

OCO asym.
Frequency $\left(\mathrm{cm}^{-1}\right)$
j) 1482

k) 1435

1) $1415-1410$

m) $1396-1393$

n) $1382-1381$

o) $1378-1373$

p) $1361-1360$

q) $1257-1255$
Predicted mode

$\mathrm{C}-\mathrm{H}$ bend

OCO sym.

OCO sym.

$\mathrm{C}-\mathrm{H}$ bend, $\mathrm{C}=\mathrm{C}$ stretch $\mathrm{C}-\mathrm{H}$ bend, $\mathrm{C}=\mathrm{C}$ stretch OCO sym., $\mathrm{C}-\mathrm{H}$ bend OCO sym., $\mathrm{C}-\mathrm{H}$ bend $\mathrm{C}-\mathrm{H}$ bend 
Predicted vibration frequencies with their vibrational modes for UiO-66:

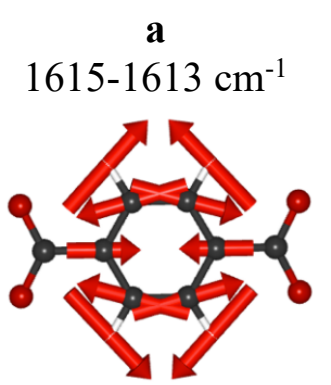

e

$1577-1576 \mathrm{~cm}^{-1}$



i

$1488 \mathrm{~cm}^{-1}$

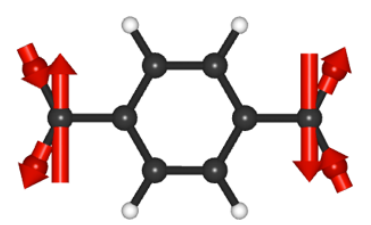

m

$1396-1393 \mathrm{~cm}^{-1}$

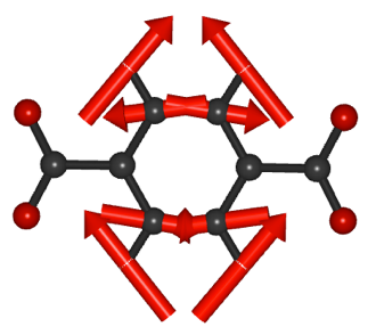

$\mathbf{q}$

$1257-1255 \mathrm{~cm}^{-1}$

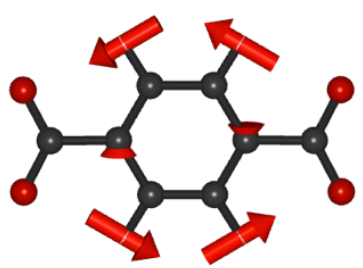

c

$1592-1591 \mathrm{~cm}^{-1}$

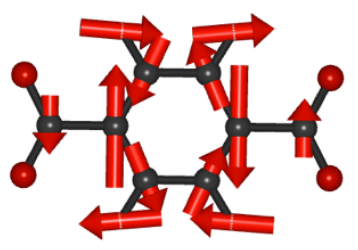

f



j

$1482 \mathrm{~cm}^{-1}$

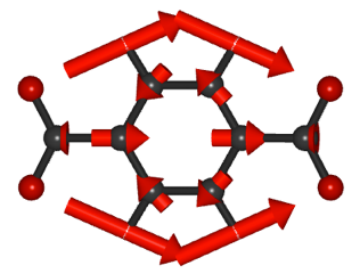

n

$1382-1381 \mathrm{~cm}^{-1}$

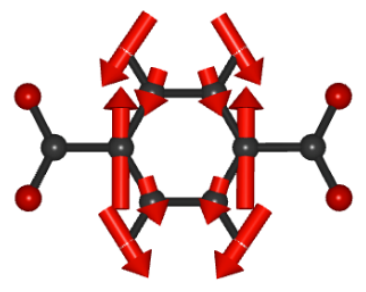

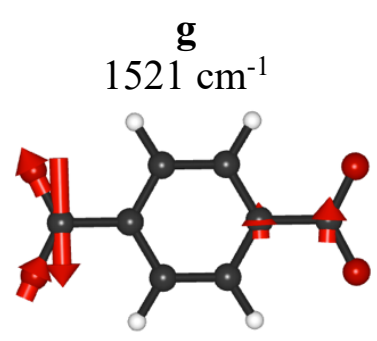

k

$1435 \mathrm{~cm}^{-1}$

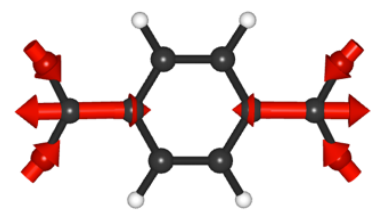

o

$1378-1373 \mathrm{~cm}^{-1}$

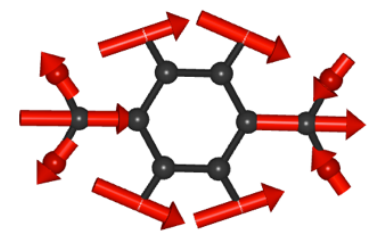

d



h

$1516 \mathrm{~cm}^{-1}$

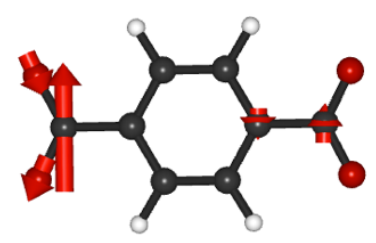

1

$1415-1410 \mathrm{~cm}^{-1}$

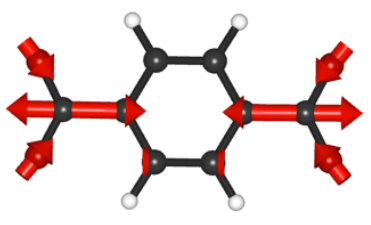

p

$1361-1360 \mathrm{~cm}^{-1}$

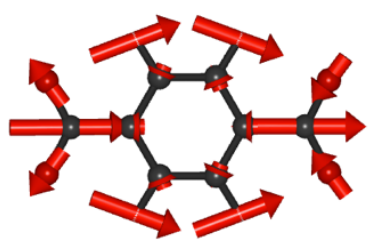

Figure S42. Summary of phonon mode calculations for UiO-66. 


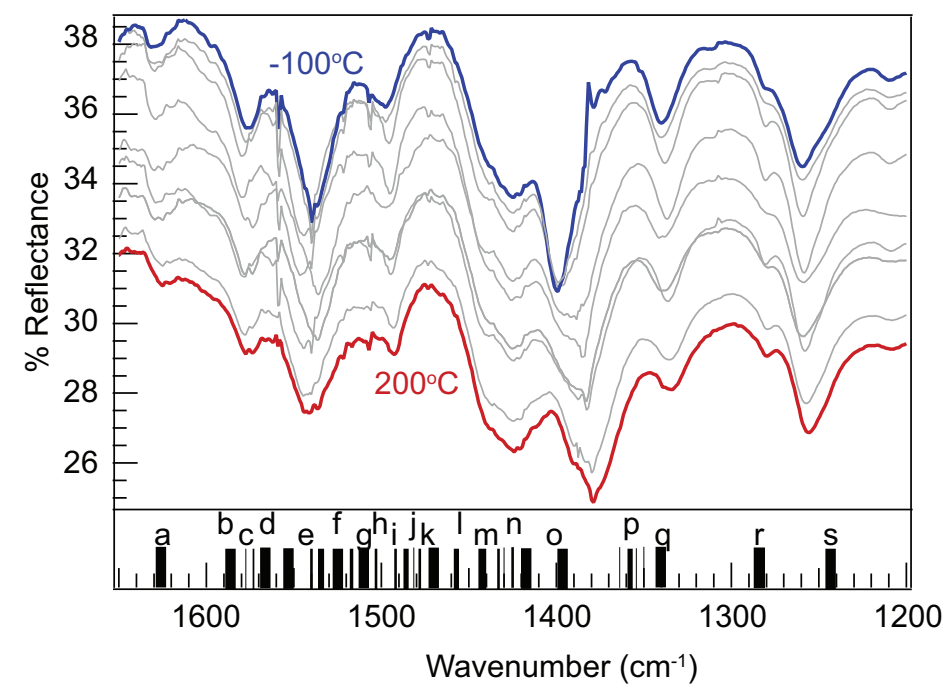

\section{Frequency Predicted mode} $\left(\mathrm{cm}^{-1}\right)$
a) $1634-1627$

b) $1594-1589$

c) 1582

c) 1578

d) $1574-1568$

e) $1562-1555$

e) $1544-1543$

e) $1540-1536$

f) $1533-1528$

f) $1521-1520$

g) $1517-1513$

h) 1507

i) 1496-1495 C-O stretch, NHN bend

j) 1490-1488 OCO asym., NHN bend

j) $1484 \quad$ OCO asym., NHN bend

j) $1481 \quad$ OCO asym., NHN bend

k) 1478-1472 C-H bend, N-H bend bend bend stretch

$\mathrm{HNH}$ bend

$\mathrm{HNH}$ bend

$\mathrm{HNH}$ bend

$\mathrm{HNH}$ bend, OCO asym.

HNH bend, OCO asym.

NHN bend

NHN bend, C-O stretch
Frequency $\left(\mathrm{cm}^{-1}\right)$

1) $1462-1460 \quad \mathrm{HNH}$ bend

1) 1453

m) $1449-1443$

OCO asym., C-H bend, N-H

m) $1437-1436$ $\mathrm{C}-\mathrm{H}$ bend, $\mathrm{N}-\mathrm{H}$ bend, $\mathrm{C}=\mathrm{C}$

p) $1362-1360$

p) 1356

p) 1351

q) $1347-1340$

r) $1289-1280$

s) $1247-1244$
HNH bend

HNH bend, O-H stretch, C-

$\mathrm{H}$ bend

HNH bend, O-H stretch, C-

$\mathrm{H}$ bend

NHN bend

NHN bend

NHN bend

NHN bend

OCO sym., N-H bend

OCO sym., $\mathrm{N}-\mathrm{H}$ bend

C-H bend, OCO sym., HNH bend

C-H bend, OCO sym., HNH bend

C-H bend, OCO sym., HNH bend

C-H bend, OCO sym., HNH bend

C-H bend, OCO sym., HNH bend

$\mathrm{C}-\mathrm{H}$ bend, $\mathrm{HNH}$ bend

$\mathrm{C}-\mathrm{H}$ bend 
Predicted vibration frequencies with their vibrational modes for MIL-125-NH2:

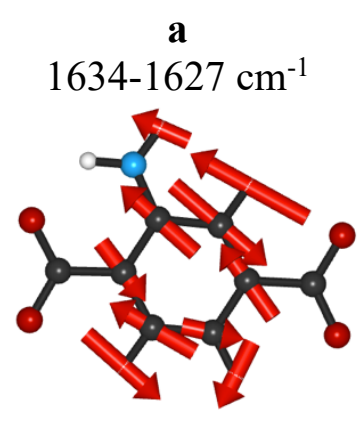

e

1562-1555,

1544-1543,

$1540-1536 \mathrm{~cm}^{-1}$

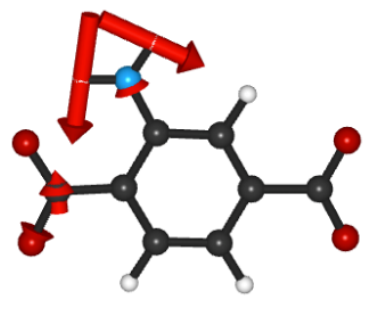

i

$1496-1495 \mathrm{~cm}^{-1}$

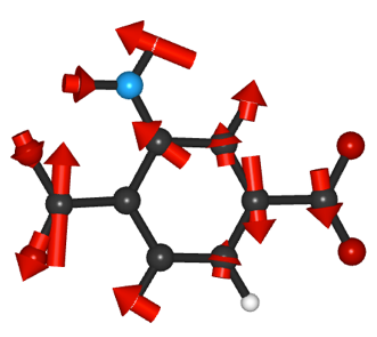

m

1449-1443,

$1437-1436 \mathrm{~cm}^{-1}$

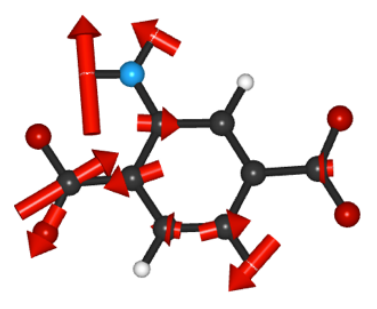

b

1594- $1589 \mathrm{~cm}^{-1}$

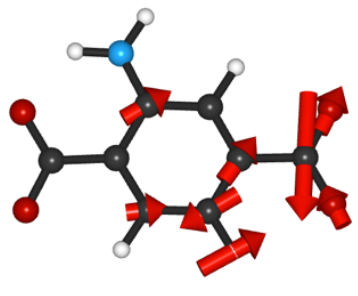

f

1533-1528, 1524,

$1521-1520 \mathrm{~cm}^{-1}$

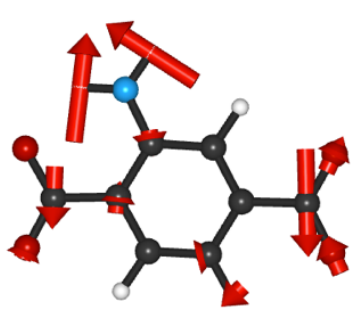

1490-1488, 1484, $1481 \mathrm{~cm}^{-1}$



n

1432, 1429, 1427, $1424-1413 \mathrm{~cm}^{-1}$

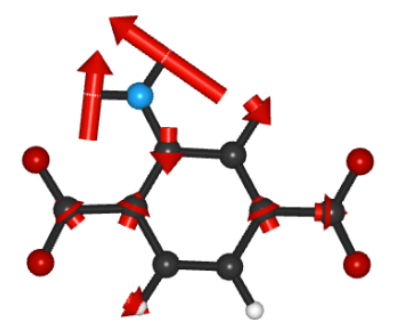

c

$1582,1578 \mathrm{~cm}^{-1}$

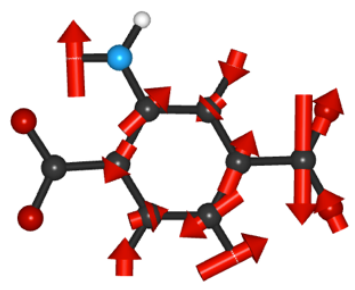

$\underset{1517-1513 \mathrm{~cm}^{-1}}{\mathbf{g}}$



k

$1478-1472 \mathrm{~cm}^{-1}$



o

1409-1408, $1403-1395 \mathrm{~cm}^{-1}$



d $1574-1568 \mathrm{~cm}^{-1}$


$1507 \mathrm{~cm}^{-1}$

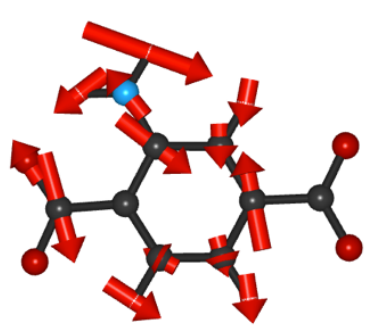

1

1462-1460, $1453 \mathrm{~cm}^{-1}$

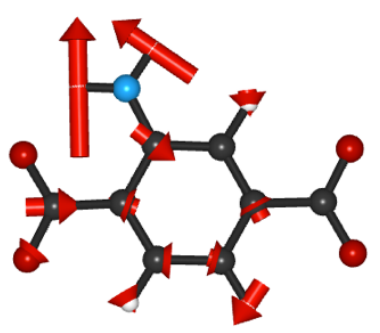

p

1366, 1362-1360, $1356,1351 \mathrm{~cm}^{-1}$

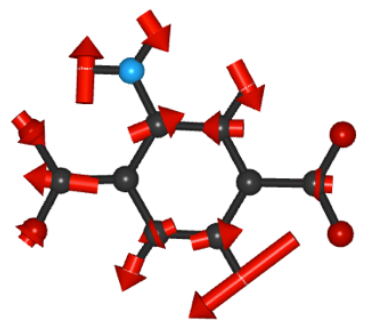




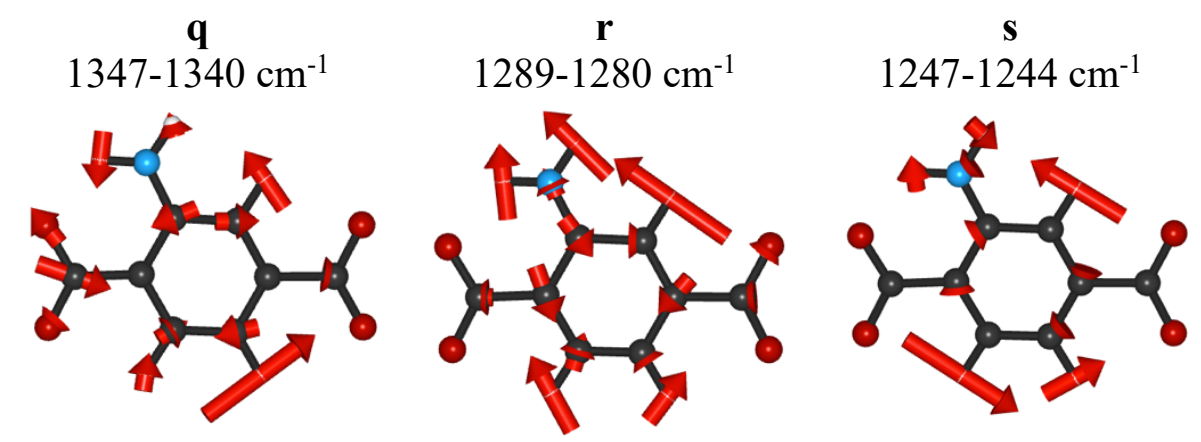

Figure S43. Summary of phonon mode calculations for MIL-125-NH2
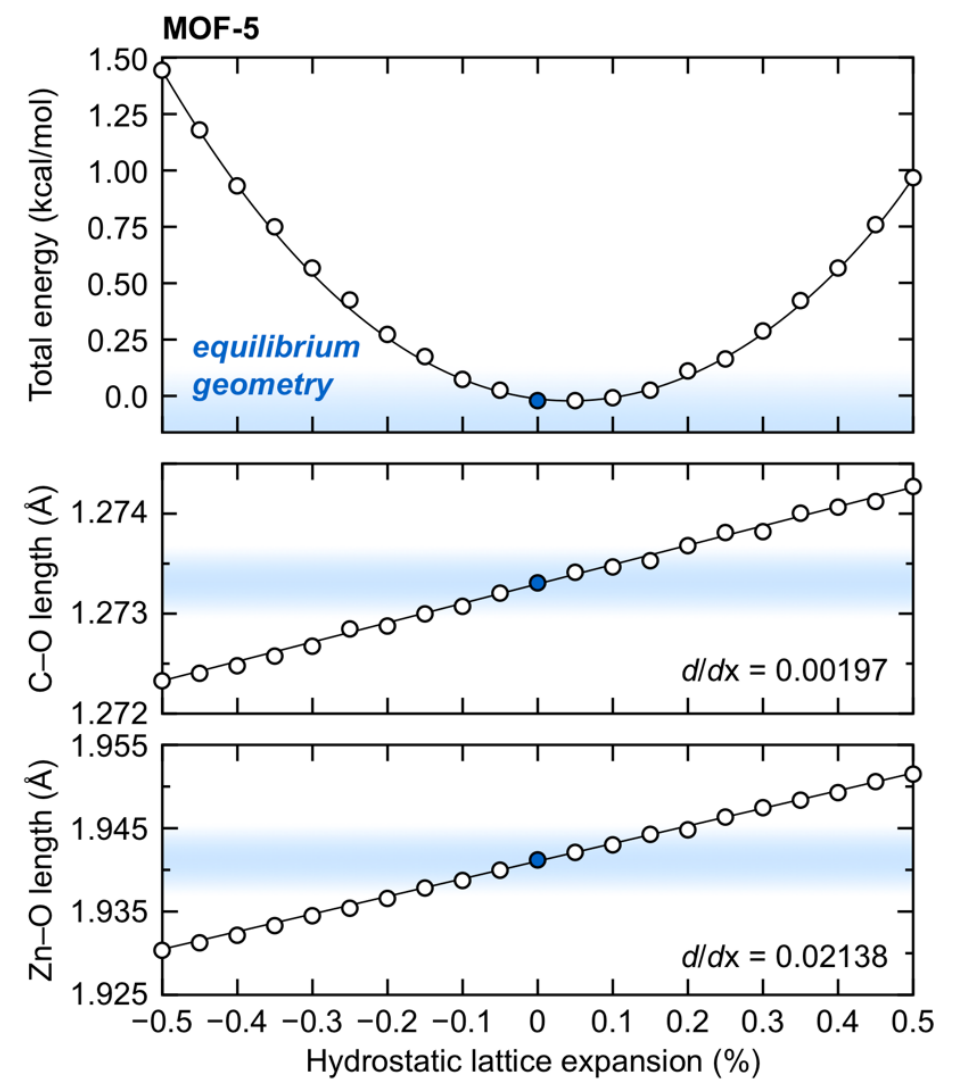

Figure S44. MOF-5 equation of state. The blue point denotes the computed equilibrium structure, but other similar energy structures are accessible (emphasized in the blue gradient). The potential energy surface is shallow around the minimum. M-O bond elongates more rapidly than the $\mathrm{C}-\mathrm{O}$ bond. 

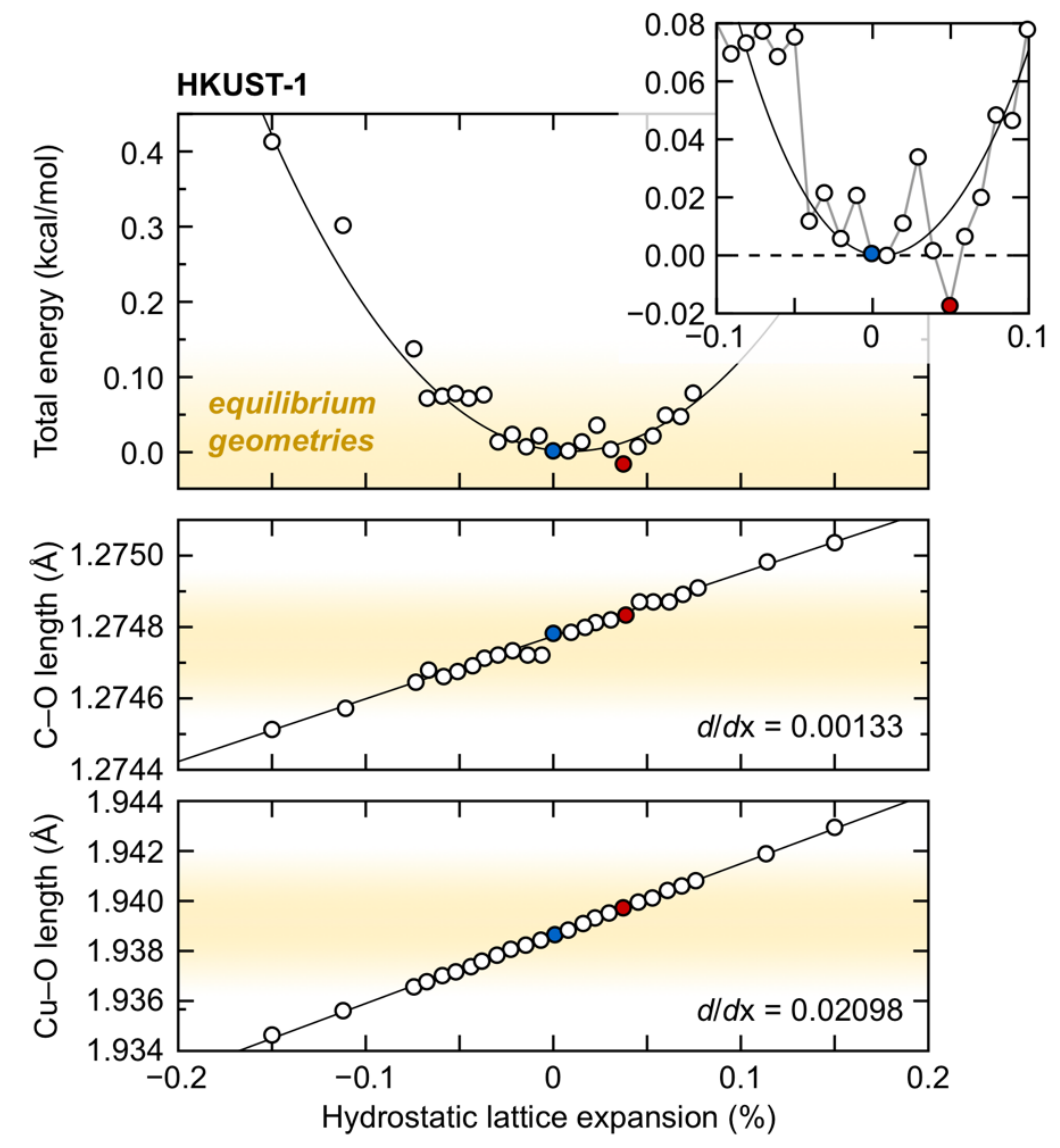

Figure S45. HKUST-1 equation of state. The blue point is the computed equilibrium structure, but other similar energy structures are accessible (emphasized in the yellow gradient). Unlike MOF-5, HKUST-1 a less smooth energy profile near the equilibrium structure. We highlight another minimum energy structure (red), which could be the "loose" geometry. Regardless, the potential energy surface is shallow around the minimum. M-O bond elongates more rapidly than the $\mathrm{C}-\mathrm{O}$ bond. 


\section{Overview of Models}

In addition to the explanation provided in the text, which we term the "Equilibrium" model, we also explored several possible alternatives based on temperature-dependent Boltzmann statistics: the "Morse Potential" and "Cross-anharmonicity" models. Here, we detail these models and provide a comparison between them.

The "Morse Potential" and "Cross-anharmonicity" models explore scenarios that are chemically different, but ask the same fundamental question of whether different Boltzmann factors between $-100{ }^{\circ} \mathrm{C}$ and $200^{\circ} \mathrm{C}$ could induce redshifts by populating states closer in energy than the fundamental transition. The "Morse Potential" model considers only the $\mathrm{C}-\mathrm{O}$ bond of a carboxylate as a Morse potential, with the well-depth, anharmonicity, and fundamental frequency taken from empirical values (see below). The "Cross-anharmonicity" model treats the C-O-M bond vector as coupled anharmonic oscillators. In both cases, however, either the Boltzmann factor produced redshifts that were too small or the energy differences between the ground and thermally populated excited vibrational states were too small. Despite the failings of these simple models, we expect that the redshifts could be explained by more complex anharmonic potential energy surfaces that treat the entire polyatomic system. Nevertheless, the "Equilibrium model" provides a complementary and convenient alternate approach. 


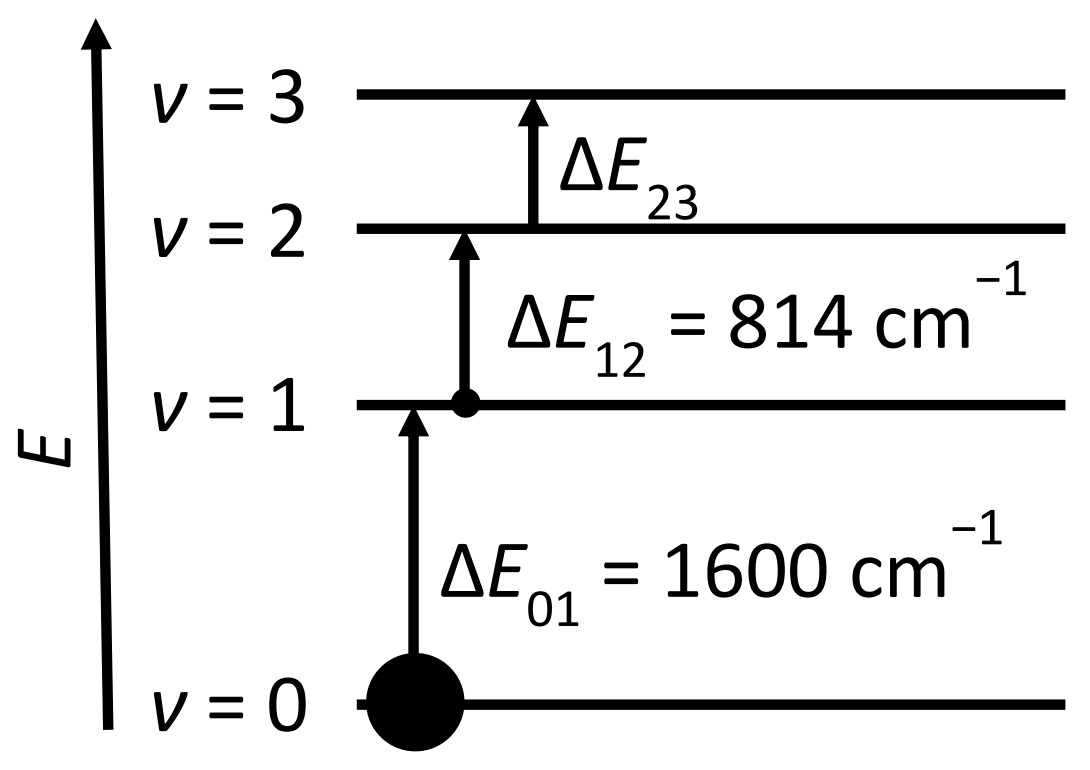

Figure S45: Simplified vibrational energy state diagram for a C-O bond. Uneven energetic spacing arises from the anharmonicity of the Morse potential. Allowed transitions are labeled with corresponding energies. Boltzmann populations are indicated qualitatively by the size of the black circles.

A Morse potential depends on two parameters $D_{e}$ and $a$, with larger values of $D_{e}$ giving deeper curves and larger $a$ giving narrower curves. According to this model, the energetic difference between adjacent levels decreases towards higher energy states, producing unevenly distributed vibrational energy levels. Hence, bonds treated with the Morse potential are termed anharmonic, in contrast to the evenly spaced states of a simple harmonic oscillator. At any given temperature, the population of each vibrational state depends on thermal energy such that the overall distribution of the ensemble across the vibrational states can be predicted from Boltzmann statistics. According to this model, sufficient thermal energy should induce redshifts to the apparent vibrational frequency by populating closely spaced excited states.

Given experimental carboxylate stretching frequencies $\left(\Delta \mathrm{E}_{01}\right)$, we determined the parameters necessary to observe an experimental redshift of about $-6 \mathrm{~cm}^{-1}$ between room temperature and 200 ${ }^{\circ} \mathrm{C}$. To simplify the calculation, we set the temperatures to be $T=173 \mathrm{~K}$ and $T^{\prime}=473 \mathrm{~K}$ and calculated the probability of the ground vibrational state $p_{0}$ and the first excited state $p_{1}$ at $T$ and $T^{\prime}$ based on a Boltzmann distribution. The energy difference between them $\left(\Delta E_{01}\right)$ was set to be a typical carboxylate stretching frequency of $1600 \mathrm{~cm}^{-1}$. We defined the apparent peak position to be $p_{0} \Delta E_{01}+p_{1} \Delta E_{12}$ at $T$, and $p_{0}{ }^{\prime} \Delta E_{01}+p_{1}{ }^{\prime} \Delta E_{12}$ at $T^{\prime}$, where $\Delta E_{12}$ denotes the energetic difference between the first and second excited states. By equating $\left(p_{0}{ }^{\prime} \Delta E_{01}+p_{1}{ }^{\prime} \Delta E_{12}\right)-\left(p_{0} \Delta E_{01}+p_{1} \Delta E_{12}\right)$ to $-6 \mathrm{~cm}^{-1}$, we can calculate $\Delta E_{12}=814 \mathrm{~cm}^{-1}$. This value is unrealistically small in comparison to $\Delta E_{01}$. Additionally, their energetic difference far exceeds the peak width of the observed carboxylate stretches, meaning that two peaks rather than one redshifted peak would be observed. Once we know $\Delta E_{01}$ and $\Delta E_{12}$, we can calculate the parameters $D_{e}=\frac{(2 \Delta E 01-\Delta E 12)^{2}}{2(\Delta E 01-\Delta E 12)}$ in Morse potential. Once we further assume the reduced mass $\mu$ to be $m_{\mathrm{C}} m_{\mathrm{O}} /\left(m_{\mathrm{C}}+m_{\mathrm{O}}\right)$, where $m_{\mathrm{C}}$ and $m_{\mathrm{O}}$ 
mean the mass of carbon atom and oxygen atom respectively, we can calculate the parameters $a=$ $\frac{2 \pi \sqrt{(\Delta E 01-\Delta E 12) \mu}}{h}$

in Morse potential. Note, however, that the carboxylate stretches constitute polyatomic systems, with at least the three $\mathrm{O}-\mathrm{C}-\mathrm{O}$ atoms instead of just two atoms $\mathrm{C}-\mathrm{O} .{ }^{12}$ With these values, we calculated $D_{\mathrm{e}}=3621 \mathrm{~cm}^{-1}$ (or $0.45 \mathrm{eV}$ ) and $a=13 \AA^{-1}$. Although, values of $D_{\mathrm{e}}$ should be near the corresponding bond enthalpy, this calculated values is an order of magnitude smaller than the $5 \mathrm{eV}$ bond energies reported for $\mathrm{C}-\mathrm{O}$ single bonds of benzoic acid. ${ }^{13}$ Furthermore, the calculated $a$ of $13 \AA^{-1}$ is much larger than literature values ranging from $0.4 \AA^{-1}$ to $5 \AA^{-1} .^{12}$ This discrepancy raises the question whether it is proper to apply a Morse potential to this polyatomic system. Thus, we suspect that this is not a convincing explanation for the redshift.

\section{Cross-anharmonicity}

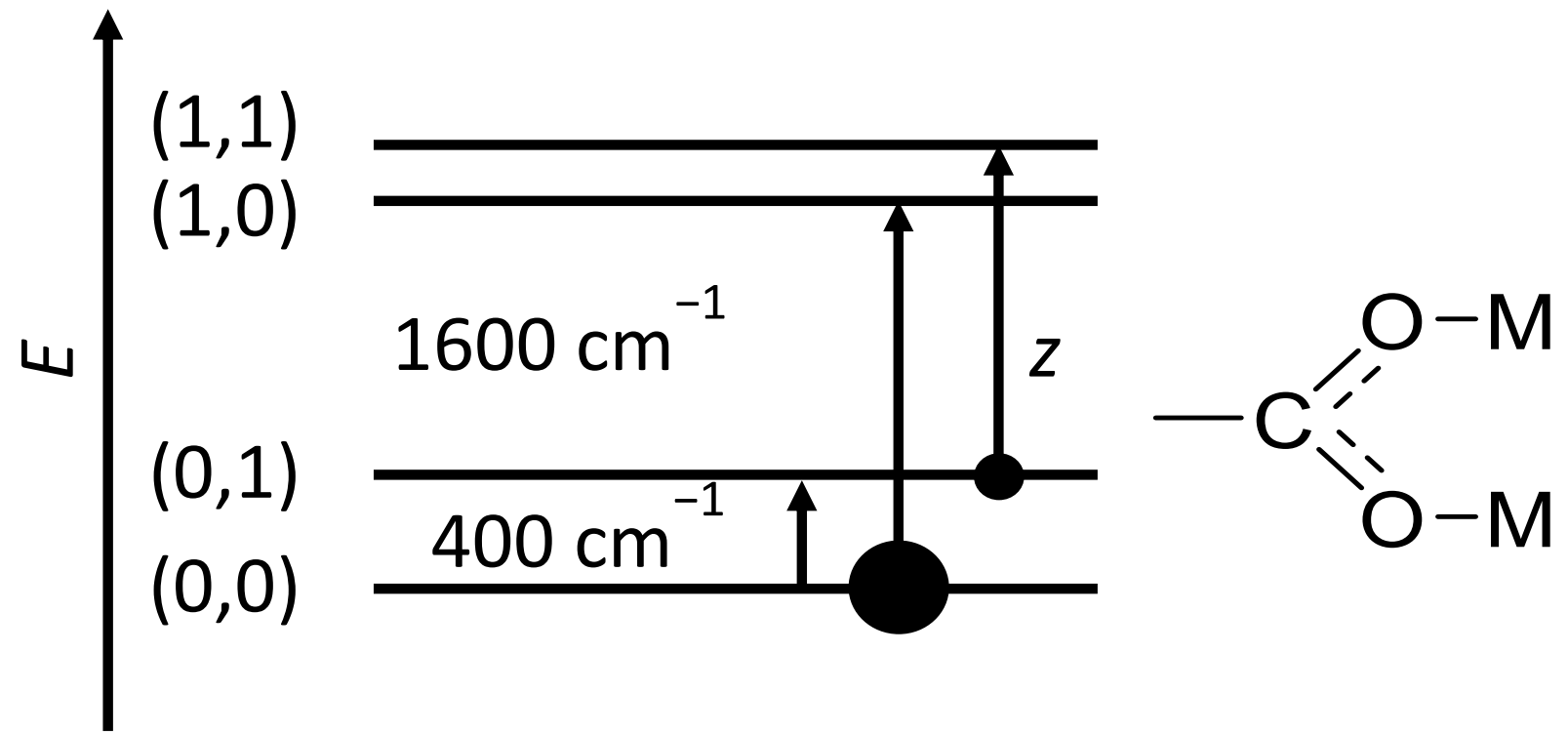

Figure S46: Schematic representation of the Cross-anharmonicity model. The fundamental frequencies of the $\mathrm{M}-\mathrm{O}$ and $\mathrm{O}-\mathrm{C}-\mathrm{O}$ bonds are denoted by $(0,0) \rightarrow(0,1)$ and $(0,0) \rightarrow(1,0)$, respectively. The $(0,1) \rightarrow(1,1)$ transition relate to coupled motion between the $\mathrm{M}-\mathrm{O}$ and $\mathrm{C}-\mathrm{O}$ fragments.

Because we monitor carboxylate stretches as indirect evidence for $\mathrm{M}-\mathrm{O}$ dynamics, we considered a "cross-anharmonicity" model describing their coupled motion. We define (number1, number2) to represent the quantum numbers of the $\mathrm{M}-\mathrm{O}$ and $\mathrm{O}-\mathrm{C}-\mathrm{O}$ fragments, respectively. Therefore, $(0,0) \rightarrow(0,1)$ and $(0,0) \rightarrow(1,0)$ correspond to the fundamental frequencies of the M-O and O-C-O stretches, respectively. Given frequencies of $1600 \mathrm{~cm}^{-1}$ for C-O and $400 \mathrm{~cm}^{-1}$ for M$\mathrm{O}$, we define $\mathrm{E}(1,0)-\mathrm{E}(0,0)$ to be $1600 \mathrm{~cm}^{-1}$ and $\mathrm{E}(0,1)-\mathrm{E}(0,0)$ to be $400 \mathrm{~cm}^{-1}$. Therefore, a redshift could arise from population of the coupled $(0,1) \rightarrow(1,1)$ transition, which must be lower in energy than $(0,0) \rightarrow(1,0)$. Using a similar Boltzmann statistical approach to the Morse Potential model, we calculate $\mathrm{E}(1,1)-\mathrm{E}(0,1)$ to be $1569 \mathrm{~cm}^{-1}$ in order to produce a redshift of $-6 \mathrm{~cm}^{-1}$. This value is $31 \mathrm{~cm}^{-1}$ smaller than $\mathrm{E}(1,0)-\mathrm{E}(0,0)$, which exceeds the experimentally observed carboxylate peak widths, which would give rise two apparent stretches rather than a single coalesced peak. 
Equilibrium Model

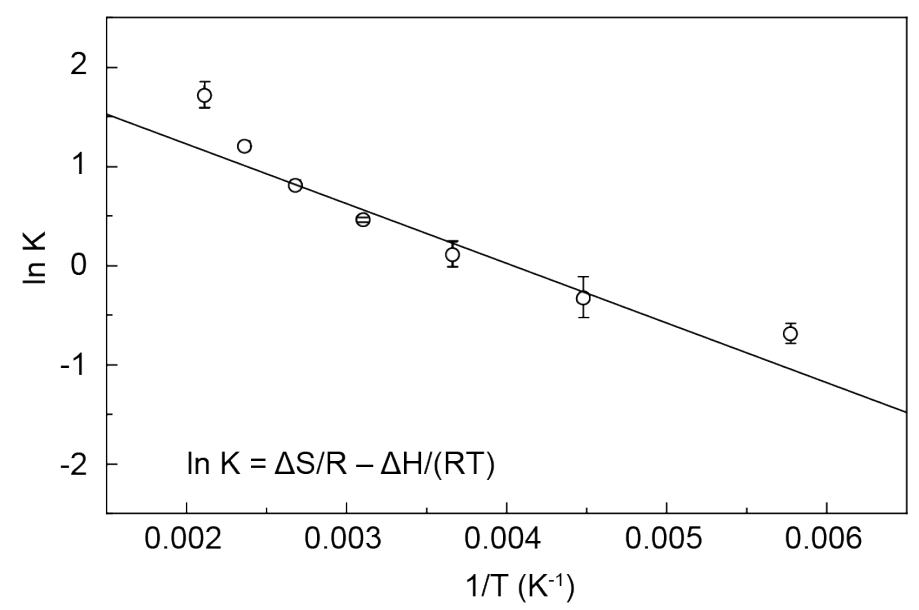

Figure S47: van 't Hoff analysis of tight and loose equilibrium species as determined from global population analysis in Figure 3. $\mathrm{K}$ is defined as [loose]/[tight]. Measurements were cycled, giving multiple data points at each temperature. Error bars were calculated based on the Gaussian fits.

As in the previous models, the Equilibrium Model explores whether the carboxylate redshifts arise from temperature-dependent intensities of two overlapping bands. Supposing an equilibrium exists as $\mathrm{A} \rightleftharpoons \mathrm{B}$ with $\mathrm{K}_{\mathrm{eq}}=[\mathrm{B}] /[\mathrm{A}]$, we assume $\mathrm{A}$ to be a "tight" $\mathrm{O}-\mathrm{M}$ state with vibrational energy $v_{A}$ and $B$ to be a "loose" state with vibrational energy $v_{B}$, and $v_{A}>v_{B}$. Rather than describing two specific metal-carboxylate configurations, the two states represent ensembles of configurations in shallow potential energy surfaces that appear more tight or loose (Scheme 2). Therefore, the equilibrium will shift to the "loose" state at higher temperatures. Since $v_{\mathrm{A}}>v_{\mathrm{B}}$, statistically, a redshift should be observed. Using $\mathrm{x}_{\mathrm{A}}$ and $\mathrm{x}_{\mathrm{B}}$ to denote the mole fraction of $\mathrm{A}$ and $\mathrm{B}, \mathrm{x}_{\mathrm{A}}=1 /\left(1+\mathrm{K}_{\mathrm{eq}}\right)$ and $\mathrm{x}_{\mathrm{B}}=\mathrm{K}_{\mathrm{eq}} /\left(1+\mathrm{K}_{\mathrm{eq}}\right)$. Similarly, we define the apparent peak position to be $\mathrm{x}_{\mathrm{A}} v_{\mathrm{A}}$ $+\mathrm{x}_{\mathrm{B}} v_{\mathrm{B}}$ at $\mathrm{T}$, and $\mathrm{x}_{\mathrm{A}} v_{\mathrm{A}}+\mathrm{x}_{\mathrm{B}^{\prime}} \mathrm{v}_{\mathrm{B}}$ at $\mathrm{T}^{\prime}$. After substituting $\mathrm{K}_{\mathrm{eq}}=\exp \left[-\Delta \mathrm{G}^{\circ} /(\mathrm{RT})\right]$, we get an expression for the redshift as a function of temperature that is fundamentally equivalent to the Boltzmann expressions in the Morse and Cross-anharmonicity models. Using a global fitting procedure, we extract $v_{\mathrm{A}}$ and $v_{\mathrm{B}}$ from our experimental data and assume that all the peaks at each temperature can be deconvoluted into the sum of two Gaussian peaks $\mathrm{A}$ and $\mathrm{B}$ with fixed frequencies and peak widths, but variable areas. Using this method, we find $v_{\mathrm{A}}=1595.8 \pm 0.1 \mathrm{~cm}^{-1}, v_{\mathrm{B}}=1587 \pm 1 \mathrm{~cm}^{-1}$, and widths $\mathrm{w}_{\mathrm{A}}=11.9 \pm 0.2 \mathrm{~cm}^{-1}$, and $\mathrm{w}_{\mathrm{B}}=13.4 \pm 0.2 \mathrm{~cm}^{-1}$. We define the equilibrium constant by the ratio of the areas $K=A_{B} / A_{A}$, where $A_{A}$ and $A_{B}$ are the areas of peak $A$ and peak $B$, respectively, and plot $\ln \mathrm{K}$ as a function of $1 / \mathrm{T}$ for a van 't Hoff analysis. Using the linear equation $\ln \mathrm{K}=\Delta \mathrm{S} / \mathrm{R}-\Delta \mathrm{H} /(\mathrm{RT})$ to fit, we can get $\Delta \mathrm{H}=5.0 \pm 0.9 \mathrm{~kJ} \mathrm{~mol}^{-1}, \Delta \mathrm{S}=20 \pm 3 \mathrm{~J} \mathrm{~mol}^{-1} \mathrm{~K}^{-1}$. The deviation from a straight line in the van 't Hoff plot inspired us to consider a non-zero heat capacity change in this reaction. Thus, we plot $\mathrm{K}$ as a function of $\mathrm{T}$ and use both $\mathrm{K}=\exp [-(\Delta \mathrm{H}-$ $\mathrm{T} \Delta \mathrm{S}) /(\mathrm{RT})]$ and $\mathrm{K}=\exp \left[-\left(\Delta \mathrm{H}_{\mathrm{R}}+\Delta \mathrm{C}_{\mathrm{P}}\left(\mathrm{T}-\mathrm{T}_{\mathrm{R}}\right)-\mathrm{T}\left(\Delta \mathrm{S}_{\mathrm{R}}+\Delta \mathrm{C}_{\mathrm{P}} \ln \left(\mathrm{T} / \mathrm{T}_{\mathrm{R}}\right)\right)\right) /(\mathrm{RT})\right]$ to fit. In the second fitting, the reference temperature $\mathrm{T}_{\mathrm{R}}$ is set to be $298.15 \mathrm{~K}$. The first fitting gives us $\Delta \mathrm{H}=5 \pm 1 \mathrm{~kJ}$ $\mathrm{mol}^{-1}, \Delta \mathrm{S}=21 \pm 3 \mathrm{~J} \mathrm{~mol}^{-1} \mathrm{~K}^{-1}$. The second fitting gives us $\Delta \mathrm{H}_{\mathrm{R}}=5.9 \pm 0.2 \mathrm{~kJ} \mathrm{~mol}^{-1}, \Delta \mathrm{S}_{\mathrm{R}}=22.0$ $\pm 0.6 \mathrm{~J} \mathrm{~mol}^{-1} \mathrm{~K}^{-1}$, and $\Delta \mathrm{C}_{\mathrm{P}}=37 \pm 4 \mathrm{~J} \mathrm{~mol}^{-1} \mathrm{~K}^{-1}$. 


\section{References}

1. Van Goethem, C.; Mertens, M.; Cirujano, F. G.; Seo, J. W.; De Vos, D.; Vankelecom, I. F. J. Improved MOF Nanoparticle Recovery and Purification Using Crosslinked PVDF Membranes. Chem. Commun. 2018, 54 (53), 7370-7373, DOI: 10.1039/C8CC04326D.

2. Castells-Gil, J.; Padial, N. M.; Almora-Barrios, N.; Albero, J.; Ruiz-Salvador, A. R.; González-Platas, J.; García, H.; Martí-Gastaldo, C. Chemical Engineering of Photoactivity in Heterometallic Titanium-Organic Frameworks by Metal Doping. Angew. Chem. Int. Ed. 2018, 57 (28), 8453-8457, DOI: 10.1002/anie.201802089.

3. Yang, D. A.; Cho, H. Y.; Kim, J.; Yang, S. T.; Ahn, W. S. $\mathrm{CO}_{2}$ Capture and Conversion Using Mg-MOF-74 Prepared by a Sonochemical Method. Energy Environ. Sci. 2012, 5 (4), 64656473, DOI: 10.1039/C1EE02234B.

4. Choi, I.; Jung, Y. E.; Yoo, S. J.; Kim, J. Y.; Kim, H. J.; Lee, C. Y.; Jang, J. H. Facile Synthesis of M-MOF-74 (M=Co, Ni, Zn) and Its Application as an Electrocatalyst for Electrochemical $\mathrm{CO}_{2}$ Conversion and $\mathrm{H}_{2}$ Production. J. Electrochem. Sci. Technol. 2017, 8 (1), 61-68, DOI: 10.5229/JECST.2017.8.1.61.

5. Kaye, S. S.; Dailly, A.; Yaghi, O. M.; Long, J. R. Impact of Preparation and Handling on the Hydrogen Storage Properties Of $\mathrm{Zn}_{4} \mathrm{O}\left(1\right.$,4-benzenedicarboxylate) ${ }_{3}$ (MOF-5). J. Am. Chem. Soc., 2007, 129, 14176-14177, DOI: 10.1021/ja076877g.

6. Dan-Hardi, M.; Serre, C.; Frot, T.; Rozes, L.; Maurin, G.; Sanchez, C.; Férey, G. A New Photoactive Crystalline Highly Porous Titanium(IV) Dicarboxylate. J. Am. Chem. Soc. 2009, 131 (31), 10857-10859, DOI: 10.1021/ja903726m.

7. Katz, M. J.; Brown, Z. J.; Colón, Y. J.; Siu, P. W.; Scheidt, K. A.; Snurr, R. Q.; Hupp, J. T.; Farha, O. K. A Facile Synthesis of UiO-66, UiO-67 and Their Derivatives. Chem. Commun. 2013, 49 (82), 9449-9451, DOI: 10.1039/C3CC46105J.

8. Rodenas, T.; Luz, I.; Prieto, G.; Seoane, B.; Miro, H.; Corma, A.; Kapteijn, F.; Llabrés I Xamena, F. X.; Gascon, J. Metal-Organic Framework Nanosheets in Polymer Composite Materials for Gas Separation. Nat. Mater. 2015, 14 (1), 48-55, DOI: 10.1038/nmat4113.

9. Calleja, G.; Sanz, R.; Orcajo, G.; Briones, D.; Leo, P.; Martínez, F. Copper-Based MOF-74 Material as Effective Acid Catalyst in Friedel-Crafts Acylation of Anisole. Catal. Today 2014, 227, 130-137, DOI: 10.1016/j.cattod.2013.11.062.

10. Kresse, G.; Furthmüller, J. Efficient iterative schemes for ab initio total-energy calculations using a plane-wave basis set. Phys. Rev. B: Condens. Matter Mater. Phys., 1996, 54, 11169 11186, DOI: 10.1103/PhysRevB.54.11169.

11. Perdew, J. P.; Ruzsinszky, A.; Csonka, G. I.; Vydrov, O. A.; Scuseria, G. E.; Constantin, L. A.; Zhou, X.; K. Burke, K. Restoring the Density-Gradient Expansion for Exchange in Solids and Surfaces. Phys. Rev. Lett., 2008, 100, 136406, DOI: 10.1103/PhysRevLett.100.136406.

12. Herzberg, G. Molecular Spectra and Molecular Structure. II, Infrared and Raman Spectra of Polyatomic Molecules.; Van Nostrand Reinhold Company, Toronto, 1979.

13. Morse, P. M. Diatomic Molecules According to the Wave Mechanics. II. Vibrational Levels. Phys. Rev. 1929, 34 (1), 57-64, DOI: 10.1103/PhysRev.34.57. 